manuscript No.

(will be inserted by the editor)

\title{
On optimal $L^{2}$ - and surface flux convergence in FEM (extended version)
}

\author{
T. Horger · J.M. Melenk • B. Wohlmuth
}

Received: date / Accepted: date

\begin{abstract}
We show that optimal $L^{2}$-convergence in the finite element method on quasi-uniform meshes can be achieved if, for some $s_{0}>1 / 2$, the boundary value problem has the mapping property $H^{-1+s} \rightarrow H^{1+s}$ for $s \in\left[0, s_{0}\right]$. The lack of full elliptic regularity in the dual problem has to be compensated by additional regularity of the exact solution. Furthermore, we analyze for a Dirichlet problem the approximation of the normal derivative on the boundary without convexity assumption on the domain. We show that (up to logarithmic factors) the optimal rate is obtained.
\end{abstract}

Keywords $L^{2}$ a priori bounds · duality argument · reentrant corners

\section{Introduction}

The finite element method (FEM) is a widely used numerical technique for approximating solutions of boundary value problems. It is based on approximating the solution by piecewise polynomials of degree $k$. In the classical case of second order elliptic equations with an $H^{1}$-coercive bilinear form, the method is of optimal convergence order in the $H^{1}$-norm. An important tool for the convergence analysis in other norms such as the $L^{2}$-norm are duality arguments ("Nitsche trick"). The textbook procedure for optimal order convergence in $L^{2}$ is to exploit full elliptic regularity for the dual problem. Conversely, this procedure suggests a loss of the optimal convergence rate in $L^{2}$ if $H^{2}$-regularity fails to hold. This occurs, for example, in polygonal domains with reentrant corners.

Nevertheless, it is possible to recover the optimal convergence rate in $L^{2}$, if the exact solution has additional regularity to compensate for the lack of full regularity of the dual problem. More precisely: In this note, we consider a setting where an

J.M. Melenk

Institut für Analysis und Scientific Computing, Technische Universität Wien

E-mail: melenk@tuwien.ac.at

T. Horger, B. Wohlmuth

Technische Universität München

E-mail: horger, wohlmuth@ma.tum.de 
elliptic shift theorem holds for both the dual and the bidual problem in the range $\left[-1,-1+s_{0}\right]$ for some $s_{0} \in(1 / 2,1]$ (see Assumption 1.1) and show that if the solution is in the Sobolev space $H^{k+1+\left(1-s_{0}\right)}$, then the extra regularity $1-s_{0}$ can be exploited to recover the optimal convergence rate in $L^{2}$ (up to a logarithmic factor in the lowest order case $k=1$ ).

In the second part of this note, we consider the convergence in $L^{2}$ of the normal derivative on the boundary. We show that the optimal rate $O\left(h^{k}\right)$ (up to a logarithmic factor in the lowest order case) can be achieved, if the solution is sufficiently smooth. The proof is based on a local error analysis of the FEM as discussed, e.g., in [23, 24]. Here, we extract error bounds for the flux on the boundary from an optimal FEM estimate on a strip of width $O(h)$ near the boundary. Although we present the convergence of the flux for an $H^{1}$-conforming discretization, the techniques are applicable to mixed methods, [17, FEM-BEM coupling, [16], and mortar and DG methods, [18,25]. In fact, the results of the present work lead to a sharpening of [18, where convexity of the domain was assumed to avoid the analysis of a suitable additional dual problem. The techniques employed here are in part similar to those developed in [18]. Nevertheless, they are also significantly different since we have opted to forego the direct use of anisotropic norms and instead rely on weighted Sobolev norms and the embedding result of Lemma 2.1.

The analysis of the optimal convergence of fluxes has attracted some attention recently. Besides our own contributions [16, 17, 18, we mention the works [2, 3, 13 ] where similar results have been obtained by different methods.

\subsection{Notation}

For bounded Lipschitz domains $\Omega \subset \mathbb{R}^{d}$ with boundary $\Gamma:=\partial \Omega$, we employ standard notation for Sobolev spaces $H^{s}(\Omega)$, [1,21. We will formulate certain regularity results in terms of Besov space: for $s>0, s \notin \mathbb{N}$, and $q \in[1, \infty]$ the Besov space $B_{2, q}^{s}(\Omega)$ is defined by interpolation (the "real" method, also known as $K$-method as described, e.g., in [21,22]) as

$$
B_{2, q}^{s}(\Omega)=\left(H^{\lfloor s\rfloor}(\Omega), H^{\lceil s\rceil}(\Omega)\right)_{\theta, q}, \quad \theta=s-\lfloor s\rfloor .
$$

Integer order Besov spaces $B_{2, q}^{n}(\Omega)$ with $n \in \mathbb{N}$ are also defined by interpolation:

$$
B_{2, q}^{n}(\Omega)=\left(H^{n-1}(\Omega), H^{n+1}(\Omega)\right)_{1 / 2, q}, \quad n \in \mathbb{N} .
$$

To give some indication of the relevance of the second parameter $q$ in the definition of the Besov spaces, we recall the following (continuous) embeddings:

$$
H^{s+\varepsilon}(\Omega) \subset B_{2,1}^{s}(\Omega) \subset H^{s}(\Omega) \subset B_{2, \infty}^{s}(\Omega) \subset H^{s-\varepsilon}(\Omega) \quad \forall \varepsilon>0 .
$$

Of importance will be the distance function $\delta_{\Gamma}$ and the regularized distance function $\widetilde{\delta}_{\Gamma}$ given by

$$
\delta_{\Gamma}(x):=\operatorname{dist}(x, \Gamma), \quad \widetilde{\delta}_{\Gamma}(x):=h+\operatorname{dist}(x, \Gamma) .
$$

Later on, the parameter $h>0$ will be the mesh size of the quasi-uniform triangulation. Also of importance will be neighborhoods $S_{D}$ of the boundary $\partial \Omega$ given by

$$
S_{D}:=\left\{x \in \Omega \mid \delta_{\Gamma}(x)<D\right\},
$$

with particular emphasis on the case $D=O(h)$. 
1.2 Model problem

We let $\Omega \subset \mathbb{R}^{d}, d \in\{2,3\}$, be a bounded Lipschitz domain with a polygonal/polyhedral boundary and let (1.3) be our model problem:

$$
-\nabla \cdot(\mathbf{A}(x) \nabla u)=f \quad \text { in } \Omega, \quad u=0 \quad \text { on } \partial \Omega .
$$

We assume that $\mathbf{A}$ and $f$ are sufficiently smooth. Moreover $\mathbf{A}$ is pointwise symmetric positive definite, and $\mathbf{A}(x) \geq \alpha_{0}$ I for some $\alpha_{0}>0$ and all $x \in \Omega$. As usual, (1.3) is understood in a weak sense, i.e., for a right-hand side $f \in\left(H_{0}^{1}(\Omega)\right)^{\prime}$ the boundary value problem (1.3) reads: Find $u \in H_{0}^{1}(\Omega)$ such that

$$
a(u, v):=\int_{\Omega} \mathbf{A} \nabla u \cdot \nabla v=\langle f, v\rangle \quad \forall v \in H_{0}^{1}(\Omega) .
$$

We denote by $T:\left(H_{0}^{1}(\Omega)\right)^{\prime} \rightarrow H_{0}^{1}(\Omega)$ the solution operator. We emphasize that the choice of boundary conditions (here: homogeneous Dirichlet boundary conditions) is not essential for our purposes. Essential, however, is the following assumption:

Assumption 1.1 There exists $s_{0} \in(1 / 2,1]$ such that the solution operator $f \mapsto$ Tf for 1.4) satisfies

$$
\|T f\|_{H^{1+s_{0}(\Omega)}} \leq C\|f\|_{\left(H_{0}^{\left.1-s_{0}(\Omega)\right)^{\prime}}\right.} \leq C\|f\|_{L^{2}(\Omega)} .
$$

Here and in the following $0<c, C<\infty$ denote generic constants that do not depend on the mesh-size but possibly depend on $s_{0}$. We also use $\lesssim$ to abbreviate $\leq C$.

Remark 1.2 The present problem is symmetric. As a consequence certain dual problems that will be needed below coincide with the primal problem. This will simplify the presentation but is not essential. Inspection of the procedure below shows that we need Assumption 1.1 for the dual problem and the bidual problem with weighted right-hand side.

Let $\mathcal{T}$ be an affine simplicial quasi-uniform triangulation of $\Omega$ with mesh size $h$ and $V_{h}:=S_{0}^{k, 1}(\mathcal{T}) \subset H_{0}^{1}(\Omega)$ the continuous space of piecewise polynomials of degree $k$. This space has the following well-known properties:

(i) Existence of a quasi-local approximation operator: The Scott-Zhang operator $I_{h}^{k}: H^{1}(\Omega) \rightarrow S^{k, 1}(\mathcal{T})$ of [20] satisfies:

- If $u \in H_{0}^{1}(\Omega)$ then $I_{h}^{k} u \in V_{h}=S_{0}^{k, 1}(\mathcal{T})$.

- $I_{h}^{k}$ is quasi-local and stable: $\left\|\nabla I_{h}^{k} u\right\|_{L^{2}(K)} \lesssim\|\nabla u\|_{L^{2}\left(\omega_{K}\right)}$, where $\omega_{K}$ is the patch of elements sharing a node with $K$.

- $I_{h}^{k}$ has approximation properties:

$$
\left\|\nabla^{j}\left(u-I_{h}^{k} u\right)\right\|_{L^{2}(K)} \lesssim h^{l+1-j}\left\|\nabla^{l+1} u\right\|_{L^{2}\left(\omega_{K}\right)}, \quad j \in\{0,1\}, \quad 0 \leq l \leq k .
$$

(ii) For every $v \in B_{2, \infty}^{3 / 2}(\Omega) \cap H_{0}^{1}(\Omega)$ there holds

$$
\inf _{z \in V_{h}}\|v-z\|_{H^{1}(\Omega)} \leq h^{1 / 2}\|v\|_{B_{2, \infty}^{3 / 2}(\Omega)} .
$$

(This follows from property (i) and an interpolation argument using the $K$ method). 
(iii) The space $V_{h}$ satisfies standard elementwise inverse estimates: for integer $0 \leq j \leq m \leq k$

$$
|v|_{H^{m}(K)} \leq C h^{-(m-j)}|v|_{H^{j}(K)} \quad \forall v \in V_{h^{\prime}} .
$$

The Galerkin method for (1.4) is then: Find $u_{h} \in V_{h}$ such that

$$
a\left(u_{h}, v\right)=\langle f, v\rangle \quad \forall v \in V_{h} .
$$

Remark 1.3 The restriction to simplicial triangulations is not essential. Our primary motivation for this restriction is that in this case the space $V_{h}$ is known to have the above approximation properties, the inverse estimates, and moreover it has the "superapproximation property" that underlies the local error analysis as presented in [24, Sec. 5.4].

\section{Regularity}

\subsection{Preliminaries}

A key mechanism in our arguments that will allow us to exploit additional regularity of a function is the following embedding theorem.

Lemma 2.1 The following estimates hold, if $\Omega \subset \mathbb{R}^{d}$ is a bounded Lipschitz domain and $z$ sufficiently regular.

$$
\begin{aligned}
\left\|\widetilde{\delta}_{\Gamma}^{-1 / 2+\varepsilon} z\right\|_{L^{2}(\Omega)} & \leq\left\|\delta_{\Gamma}^{-1 / 2+\varepsilon} z\right\|_{L^{2}(\Omega)} \leq C_{\varepsilon}\|z\|_{H^{1 / 2-\varepsilon}(\Omega)}, \quad \varepsilon \in(0,1 / 2], \\
\left\|\widetilde{\delta}_{\Gamma}^{-1 / 2} z\right\|_{L^{2}(\Omega)} & \leq C|\ln h|^{1 / 2}\|z\|_{B_{2,1}^{1 / 2}(\Omega)}, \\
\left\|\widetilde{\delta}_{\Gamma}^{-1 / 2-\varepsilon} z\right\|_{L^{2}(\Omega)} & \leq C_{\varepsilon} h^{-\varepsilon}\|z\|_{B_{2,1}^{1 / 2}(\Omega)}, \quad \varepsilon>0 \\
\|z\|_{L^{2}\left(S_{h}\right)} & \leq C h^{1 / 2}\|z\|_{B_{2,1}^{1 / 2}(\Omega)}, \quad h>0 \\
\|z\|_{L^{2}(\Gamma)} & \leq C\|z\|_{B_{2,1}^{1 / 2}(\Omega)}
\end{aligned}
$$

Proof The estimate involving $\delta_{\Gamma}$ in (2.1) can be found, e.g., in [10, Thm. 1.4.4.3] and (2.4) is shown in [14, Lemma 2.1]. The estimates (2.2), (2.3), (2.5) follow from 1D Sobolev embedding theorems for $L^{\infty}$ and locally flattening the boundary $\Gamma$ in the same way as it is done in the proof of [14, Lemma 2.1]. For example, for (2.5) we note that a local flattening of the boundary $\Gamma$ and the $1 \mathrm{D}$ embedding $\|v\|_{L^{\infty}(0,1)}^{2} \lesssim\|v\|_{L^{2}(0,1)}\|v\|_{H^{1}(0,1)}$ imply $\|z\|_{L^{2}(\Gamma)}^{2} \lesssim\|z\|_{L^{2}(\Omega)}\|z\|_{H^{1}(\Omega)}$. This implies the estimate $\|z\|_{L^{2}(\Gamma)} \lesssim\|z\|_{B_{2,1}^{1 / 2}(\Omega)}$ by [21, Lemma 25.3]. We recall that for half-spaces, the upper bound (2.5) can be directly found in [22, Thm. 2.9.3], see also the comment in [21, Sec. 32, Eq. (32.8)].

One of several applications of Lemma 2.1 is that it allows us to transform negative norms into weighted $L^{2}$-estimates: 
Lemma 2.2 For $\varepsilon \in(0,1 / 2]$ and sufficiently regular $z$ there holds

$$
\begin{aligned}
\left\|\delta_{\Gamma}^{\beta} z\right\|_{\left(H^{1 / 2-\varepsilon}(\Omega)\right)^{\prime}} & \leq C_{\varepsilon}\left\|\delta_{\Gamma}^{\beta+1 / 2-\varepsilon} z\right\|_{L^{2}(\Omega)}, \quad-1<\beta \leq 0 \\
\left\|\widetilde{\delta}_{\Gamma}^{-1} z\right\|_{\left(B_{2,1}^{1 / 2}(\Omega)\right)^{\prime}} & \leq C|\ln h|^{1 / 2}\left\|\widetilde{\delta}_{\Gamma}^{-1 / 2} z\right\|_{L^{2}(\Omega)} .
\end{aligned}
$$

Proof Firstly, we show (2.6):

$$
\begin{aligned}
\left\|\delta_{\Gamma}^{\beta} z\right\|_{\left(H^{1 / 2-\varepsilon}(\Omega)\right)^{\prime}} & =\sup _{v \in H^{1 / 2-\varepsilon}(\Omega)} \frac{\left\langle\delta_{\Gamma}^{\beta} z, v\right\rangle}{\|v\|_{H^{1 / 2-\varepsilon}(\Omega)}} \\
& =\sup _{v \in H^{1 / 2-\varepsilon}(\Omega)} \frac{\left\langle\delta_{\Gamma}^{\beta+1 / 2-\varepsilon} z, \delta_{\Gamma}^{-1 / 2+\varepsilon} v\right\rangle}{\|v\|_{H^{1 / 2-\varepsilon}(\Omega)}} \leq C_{\varepsilon}\left\|\delta_{\Gamma}^{\beta+1 / 2-\varepsilon} z\right\|_{L^{2}(\Omega)},
\end{aligned}
$$

where, in the last step, we employed (2.1) of Lemma 2.1. Secondly, 2.7) follows by the same type of arguments, where the application of (2.1) is replaced with that of (2.2).

\subsection{Regularity}

We recall the following variant of interior regularity of elliptic problems:

Lemma 2.3 Let $\Omega$ be a bounded Lipschitz domain and $z \in H^{1+\beta}(\Omega), \beta \in(0,1]$, solve

$$
-\nabla \cdot(\mathbf{A} \nabla z)=f \quad \text { in } \Omega .
$$

Then, for a constant $C>0$ depending only on $\|\mathbf{A}\|_{C^{0,1}(\bar{\Omega})}, \alpha_{0}, \beta$, and $\Omega$

$$
\left\|\delta_{\Gamma}^{1-\beta} \nabla^{2} z\right\|_{L^{2}(\Omega)} \leq C\left(\left\|\delta_{\Gamma}^{1-\beta} f\right\|_{L^{2}(\Omega)}+\|z\|_{H^{1+\beta}(\Omega)}\right) .
$$

Proof The upper bound follows from local interior regularity for elliptic problems (see [19, Lemma 5.7.2] or [9, Thm. 8.8]) and a Besicovitch covering argument, see, e.g., [6, Section 1.5.2] and [15, Chapter 5]. We refer also to [12, Lemma A.3] where a closely related result is worked out in detail.

\subsubsection{Refined regularity for polygons and polyhedra}

It is worth pointing out that neither the structure of the boundary $\Gamma$ nor the kind of boundary conditions play a role in Lemma 2.3. One possible interpretation of Lemma 2.3 is that $z$ could lose the $H^{2}$-regularity anywhere near $\Gamma$. For certain boundary conditions such as homogeneous Dirichlet conditions and piecewise smooth geometries $\Gamma$ the solution fails to be in $H^{2}$ only near the points of nonsmoothness of the geometry. With methods similar to those of Lemma 2.3 one can show the following, stronger result:

Lemma 2.4 Let $\Omega$ be a (curvilinear) polygon in $2 D$ or a (curvilinear) polyhedron in $3 D$. Denote by $\mathcal{E}$ the set of all vertices of $\Omega$ in $2 D$ and the set of all edges of $\Omega$ in $3 D$. Let $\delta_{\mathcal{E}}$ be the distance from $\mathcal{E}$. Let $z \in H^{1+\beta}(\Omega), \beta \in(0,1]$, solve [1.3). Then, for a constant $C$ depending only on $\alpha_{0},\|\mathbf{A}\|_{C^{0,1}(\bar{\Omega})}, \beta$, and $\Omega$,

$$
\left\|\delta_{\mathcal{E}}^{1-\beta} \nabla^{2} z\right\|_{L^{2}(\Omega)} \leq C_{\beta}\left(\left\|\delta_{\mathcal{E}}^{1-\beta} f\right\|_{L^{2}(\Omega)}+\|z\|_{H^{1+\beta}(\Omega)}\right) .
$$


Proof Follows from local considerations as in Lemma 2.3. The novel aspect is the behavior near the boundary away from the vertices (in 2D) and the edges (in $3 \mathrm{D})$. This is achieved with an adapted covering theorem of the type described in Theorems A.5 A.6. The key feature of these coverings is that they allow us to reduce the considerations to balls $B=B_{r}(x)$ and stretched balls $\widehat{B}=B_{(1+\varepsilon) r}(x)$ (with fixed $\varepsilon>0$ ) with $r \sim \operatorname{dist}(x, \mathcal{E})$ and the following properties: either $x \in \Omega$ with $\widehat{B}_{r}(x) \subset \Omega$ or $x \in \Gamma$ and $\widehat{B} \cap \Omega$ is a half-ball. Local elliptic regularity assertions can then be employed for each ball $B$.

Lemma 2.4 assumes that a loss of $H^{2}$-regularity occurs at any point of nonsmoothness of $\Gamma$. However, the set of "singular" vertices or edges can be further reduced. For example, in $2 \mathrm{D}$ for $\mathbf{A}=\mathrm{Id}$, it is well-known that only the vertices of $\Omega$ with interior angle greater than $\pi$ lead to a loss of full $H^{2}$-regularity. It will therefore be useful to introduce the closed set $M_{s}$ of boundary points associated with a loss of $H^{2}$-regularity. Before introducing this set, we point out that this set is a subset of the vertices and edges:

Definition 2.5 ( $H^{2}$-regular part and singular part of the boundary) Let $\Omega$ be a polygon (in 2D) or a polyhedron (in 3D) with vertices $\mathcal{A}$ and edges $\mathcal{E}$.

1. A vertex $A \in \mathcal{A}$ of $\Omega$ is said to be $H^{2}$-regular, if there is a ball $B_{\varepsilon}(A)$ of radius $\varepsilon>0$ such that the solution $u$ of (1.3) satisfies $\left.u\right|_{B_{\varepsilon}(A) \cap \Omega} \in H^{2}(\Omega)$ whenever $f \in L^{2}(\Omega)$ together with the a priori estimate $\|u\|_{H^{2}\left(B_{\varepsilon}(A) \cap \Omega\right)} \leq C\|f\|_{L^{2}(\Omega)}$.

2. In $3 D$, an edge $e \in \mathcal{E}$ of $\Omega$ with endpoints $A_{1}, A_{2}$ is said to be $H^{2}$-regular if the following condition is satisfied: There is $c>0$ such that for the neighborhood $S=\cup_{x \in e} B_{c \operatorname{dist}\left(x,\left\{A_{1}, A_{2}\right\}\right)}(x)$ of the edge $e$ we have the regularity assertion $\left.u\right|_{S \cap \Omega} \in H^{2}$ for the solution $u$ of (1.3) whenever $f \in L^{2}(\Omega)$ together with the a priori estimate $\|u\|_{H^{2}(S \cap \Omega)} \leq C\|f\|_{L^{2}(\Omega)}$.

Denote by $\mathcal{A}_{r} \subset \mathcal{A}$ the set of $H^{2}$-regular vertices and by $\mathcal{E}_{r} \subset \mathcal{E}$ the set of $H^{2}$ regular edges. Correspondingly, let $\mathcal{A}_{s}:=\mathcal{A} \backslash \mathcal{A}_{r}$ and $\mathcal{E}_{s}:=\mathcal{E} \backslash \mathcal{E}_{r}$ be the set of vertices and edges, respectively, associated with a loss of $H^{2}$-regularity. Define the singular set $M_{s}$ as

$$
M_{s}:=\mathcal{A}_{s} \bigcup \mathcal{E}_{s} \subset \Gamma
$$

With the notion of the singular set in hand, we can formulate the following regularity result:

Lemma 2.6 Let $\Omega$ be a polygon or a polyhedron. Let $M_{s}$ be the singular set as defined in Definition 2.5. Then the following is true for any solution $z \in H_{0}^{1}(\Omega)$ of (1.3): If $z \in H^{1+\beta}(\Omega)$ for some $\beta \in(0,1]$, then with $\delta_{M_{s}}:=\operatorname{dist}\left(\cdot, M_{s}\right)$, there holds for some $C>0$ depending only on $\alpha_{0},\|\mathbf{A}\|_{C^{0,1}(\bar{\Omega})}, \beta$, and $\Omega$,

$$
\left\|\delta_{M_{s}}^{1-\beta} \nabla^{2} z\right\|_{L^{2}(\Omega)} \leq C_{\beta}\left(\left\|\delta_{M_{s}}^{1-\beta} f\right\|_{L^{2}(\Omega)}+\|z\|_{H^{1+\beta}(\Omega)}\right) .
$$

Proof The proof is based on local considerations as in Lemma 2.4. We recall that not all vertices and edges (in $3 \mathrm{D}$ ) are included in the singular set $M_{s}$. This is accounted for by a further refinement of the covering employed. We restrict ourselves to the 3D situation. Using finite coverings provided by Theorem A.6. one may restrict the attention to balls $B_{r}=B_{r}(x)$ and stretched balls $\widehat{B}=B_{(1+\varepsilon) r}(x)$ (with fixed $\varepsilon>0)$ with $r \sim \operatorname{dist}\left(x, M_{s}\right)$ where one of the following additional properties 
is satisfied: a) $x \in \Omega$ with $\widehat{B} \subset \Omega$; b) $x \in \mathcal{A}_{r}$ and $\widehat{B} \cap \Omega$ is a solid angle; c) $x \in \cup \mathcal{E}_{r}$ and $\widehat{B} \cap \Omega$ is a dihedral angle; d) $x$ lies in the interior of a face and $\widehat{B} \cap \Omega$ is a half-ball. We emphasize that we do not need to consider balls $B_{r}(x)$ with $x \in \mathcal{A}_{s}$ or $x \in \mathcal{E}_{s}$ since the covering provided by Theorem A.6 is such that for every such $x$ there is a neighborhood $\mathcal{U}_{x}$ of $x$ that is covered by (countably many) balls whose radii tend to 0 as their centers approach $x$.

\subsubsection{Shift theorems for locally supported right-hand sides}

We have the following continuity results for the solution operator $T$ for our model problem (1.3):

Lemma 2.7 Let Assumption 1.1 be valid. Then $T:\left(H_{0}^{1}(\Omega)\right)^{\prime} \rightarrow H_{0}^{1}(\Omega)$ satisfies

$$
\begin{aligned}
\|T f\|_{B_{2, \infty}^{3 / 2}(\Omega)} & \leq C\|f\|_{\left(B_{2,1}^{1 / 2}(\Omega)\right)^{\prime}} \\
\|T f\|_{H^{3 / 2+\varepsilon}(\Omega)} & \leq C_{\varepsilon}\left\|\delta_{\Gamma}^{1 / 2-\varepsilon} f\right\|_{L^{2}(\Omega)}, \quad 0<\varepsilon \leq s_{0}-1 / 2 .
\end{aligned}
$$

In particular, if $f \in L^{2}(\Omega)$ with $\operatorname{supp} f \subset \overline{S_{h}}$, then

$$
\begin{aligned}
\|T f\|_{B_{2, \infty}^{3 / 2}(\Omega)} & \leq C h^{1 / 2}\|f\|_{L^{2}(\Omega)}, \\
\|T f\|_{H^{3 / 2+\varepsilon}(\Omega)} & \leq C_{\varepsilon} h^{1 / 2-\varepsilon}\|f\|_{L^{2}(\Omega)}, \quad 0<\varepsilon \leq s_{0}-1 / 2 .
\end{aligned}
$$

Proof We follow the arguments of [18, Lemma 5.2]. The starting point for the proof of (2.9) is that interpolation and Assumption 1.1 yield with $\theta \in(0,1)$

$$
T:\left(\left(H_{0}^{1-s_{0}}(\Omega)\right)^{\prime},\left(H_{0}^{1}(\Omega)\right)^{\prime}\right)_{\theta, \infty} \rightarrow\left(H^{1+s_{0}}(\Omega), H^{1}(\Omega)\right)_{\theta, \infty}=B_{2, \infty}^{1+s_{0}(1-\theta)}(\Omega) .
$$

Next, we recognize as in [18, Lemma 5.2] (cf. [22, Thm. 1.11.2] or [21, Lemma 41.3])

$$
\begin{aligned}
\left(\left(H_{0}^{1-s_{0}}(\Omega)\right)^{\prime},\left(H_{0}^{1}(\Omega)\right)^{\prime}\right)_{\theta, \infty} & =\left(\left(H_{0}^{1-s_{0}}(\Omega), H_{0}^{1}(\Omega)\right)_{\theta, 1}\right)^{\prime} \\
& \supset\left(\left(H^{1-s_{0}}(\Omega), H^{1}(\Omega)\right)_{\theta, 1}\right)^{\prime}=\left(B_{2,1}^{1-s_{0}(1-\theta)}(\Omega)\right)^{\prime} .
\end{aligned}
$$

Setting $\theta=1-1 /\left(2 s_{0}\right) \in(0,1 / 2]$, we get $\left(B_{2,1}^{1-s_{0}(1-\theta)}(\Omega)\right)^{\prime}=\left(B_{2,1}^{1 / 2}(\Omega)\right)^{\prime}$ and $B_{2, \infty}^{1+s_{0}(1-\theta)}(\Omega)=B_{2, \infty}^{3 / 2}(\Omega)$. The assertion (2.10) follows from the Assumption 1.1 and (2.6) with $\beta=0$. For the bound (2.11), we argue as in the proof of Lemma 2.2 and use (2.4), see also [18, Lemma 5.2]. Finally, the proof of (2.12) follows from (2.10) and the assumed support properties of $f$.

We will also require mapping properties of the solution operator $T$ in weighted spaces:

Lemma 2.8 Let Assumption 1.1 be valid. Then for $v \in L^{2}(\Omega)$

$$
\begin{aligned}
\left\|T\left(\widetilde{\delta}_{\Gamma}^{-1} v\right)\right\|_{B_{2, \infty}^{3 / 2}(\Omega)} & \leq C|\ln h|^{1 / 2}\left\|\widetilde{\delta}_{\Gamma}^{-1 / 2} v\right\|_{L^{2}(\Omega)}, \\
\left\|T\left(\widetilde{\delta}_{\Gamma}^{-1} v\right)\right\|_{H^{3 / 2+\varepsilon}(\Omega)} & \leq C_{\varepsilon} h^{-\varepsilon}\left\|\widetilde{\delta}_{\Gamma}^{-1 / 2} v\right\|_{L^{2}(\Omega)}, \quad \varepsilon \in\left(0, s_{0}-1 / 2\right], \\
\left\|T\left(\delta_{\Gamma}^{-1+2 \varepsilon} v\right)\right\|_{H^{3 / 2+\varepsilon}(\Omega)} & \leq C_{\varepsilon}\left\|\delta_{\Gamma}^{-1 / 2+\varepsilon} v\right\|_{L^{2}(\Omega)}, \quad \varepsilon \in\left(0, s_{0}-1 / 2\right] .
\end{aligned}
$$


Proof The results follow by combining Lemmas 2.2 and 2.7

For the analysis of the FEM error on the neighborhood $S_{h}$, we need a refined version of interior regularity for elliptic problems. The following result is very similar to [18. Lemma 5.4] and closely related to Lemma 2.3.

Lemma 2.9 Let $z$ solve the equation

$$
-\nabla \cdot(\mathbf{A} \nabla z)=v \quad \text { in } \Omega \text {. }
$$

Then there exist $C_{A}$ (depending only on $\alpha_{0},\|\mathbf{A}\|_{C^{0,1}(\bar{\Omega})}$ and $\Omega$ ) and $c_{1}>0$ (depending only on $\Omega$ ) such that for $z \in B_{2, \infty}^{3 / 2}(\Omega)$, we have

$$
\left\|\delta_{\Gamma}^{1 / 2} \nabla^{2} z\right\|_{L^{2}\left(\Omega \backslash S_{h}\right)} \leq C_{A}\left[\sqrt{|\ln h|}\|z\|_{B_{2, \infty}^{3 / 2}(\Omega)}+\left\|\sqrt{\delta_{\Gamma}} v\right\|_{L^{2}\left(\Omega \backslash S_{c_{1} h}\right)}\right] .
$$

If the right-hand side $v$ satisfies additionally $\operatorname{supp} v \subset \overline{S_{h}}$ and furthermore $z=T v$, then there are constants $C_{A}$ (depending only on $\alpha_{0},\|\mathbf{A}\|_{C^{0,1}(\bar{\Omega})}$, and $\Omega$ ) and $c>1$, $\tilde{c}>c^{\prime}>1$ (depending only on $\Omega$ ) such that for all sufficiently small $h>0$ :

(i) If $z \in B_{2, \infty}^{3 / 2}(\Omega)$ then $\left\|\delta_{\Gamma}^{1 / 2} \nabla^{2} z\right\|_{L^{2}\left(\Omega \backslash S_{\tilde{c} h}\right)} \leq C_{A} \sqrt{|\ln h|}\|z\|_{B_{2, \infty}^{3 / 2}(\Omega)}$.

(ii) For every $\alpha>0$ there holds

$$
\left\|\delta_{\Gamma}^{\alpha} \nabla^{3} z\right\|_{L^{2}\left(\Omega \backslash S_{\tilde{c} h}\right)} \leq C_{A}\left[\left\|\delta_{\Gamma}^{\alpha-1} \nabla^{2} z\right\|_{L^{2}\left(\Omega \backslash S_{c^{\prime} h}\right)}+\|\mathbf{A}\|_{C^{1,1}(\Omega)}\left\|\delta_{\Gamma}^{\alpha} \nabla z\right\|_{L^{2}\left(\Omega \backslash S_{c^{\prime} h}\right)}\right] .
$$

(iii) If $z \in H^{3 / 2+\varepsilon}(\Omega)$ for some $\varepsilon \in(0,1 / 2)$, then for some $C_{A, \varepsilon}>0$ (depending on $\alpha_{0},\|\mathbf{A}\|_{C^{0,1}(\bar{\Omega})}, \Omega$, and $\varepsilon$ ) there holds

$$
\left\|\nabla^{2} z\right\|_{L^{2}\left(\Omega \backslash S_{\tilde{c} h}\right)} \leq C_{A, \varepsilon} h^{-1 / 2+\varepsilon}\|z\|_{H^{3 / 2+\varepsilon}(\Omega)} .
$$

(iv) If Assumption 1.1 is valid, then for some $C>0$ depending only on the solution operator $T$ and $C_{A, \varepsilon}$ of (iii) we have $\left\|\nabla^{2} z\right\|_{L^{2}\left(\Omega \backslash S_{\tilde{c} h}\right)} \leq C\|v\|_{L^{2}(\Omega)}$.

Proof of (2.16), (2), [i2): [18, Lemma 5.4] is formulated for $-\Delta$. However, the essential property of the differential operator $\Delta$ that is required is just interior regularity. Hence, the result also stands for the present, more general elliptic operator (with the appropriate modifications due to the fact that the coefficient $\mathbf{A}$ is allowed to be non-constant). In the interest of generality, we have also tracked in (2.16) the dependence on the right-hand side $v$, which was not done in 18 , Lemma 5.4]. A full proof can be found in Appendix C

Proof of (iii): This follows again by local considerations similar to those employed in the proof of [18, Lemma 5.4] and the obvious bound $\delta_{\Gamma} \geq h$ on $\Omega \backslash S_{\tilde{c} h}$. A full proof can be found in Appendix C.

Proof of (iv): In view of (iii), we have to estimate $\|z\|_{H^{3 / 2+\varepsilon}(\Omega)}$. By the support properties of $v$, the bound (2.12) yields $\|z\|_{H^{3 / 2+\varepsilon}(\Omega)} \leq C h^{1 / 2-\varepsilon}\|v\|_{L^{2}(\Omega)}$. Inserting this in (iii) gives the result. 


\section{FEM $L^{2}$-error analysis}

Let $u_{h}$ be the FEM approximation and denote by $e=u-u_{h}$ the FEM error. The standard workhorse is the Galerkin orthogonality

$$
a(e, v)=a\left(u-u_{h}, v\right)=0 \quad \forall v \in V_{h} .
$$

We start with a weighted $L^{2}$-error:

Lemma 3.1 Let Assumption 1.1 be valid. Assume that a function $z \in H_{0}^{1}(\Omega)$ satisfies the Galerkin orthogonality

$$
a(z, v)=0 \quad \forall v \in V_{h} .
$$

Then

$$
\begin{aligned}
\left\|\delta_{\Gamma}^{-1 / 2+\varepsilon} z\right\|_{L^{2}(\Omega)} & \leq C_{\varepsilon} h^{1 / 2+\varepsilon}\|z\|_{H^{1}(\Omega)}, \quad \varepsilon \in\left(0, s_{0}-1 / 2\right], \\
\left\|\widetilde{\delta}_{\Gamma}^{-1 / 2} z\right\|_{L^{2}(\Omega)} & \leq C h^{1 / 2}|\ln h|^{1 / 2}\|z\|_{H^{1}(\Omega)} .
\end{aligned}
$$

Proof The proof follows standard lines. Define $\psi=T\left(\delta_{\Gamma}^{-1+2 \varepsilon} z\right)$, which solves

$$
\left\langle v, \delta_{\Gamma}^{-1+2 \varepsilon} z\right\rangle=a(v, \psi) \quad \forall v \in H_{0}^{1}(\Omega) .
$$

Then we have by Galerkin orthogonality for arbitrary $I \psi \in V_{h}$

$$
\left\|\delta_{\Gamma}^{-1 / 2+\varepsilon} z\right\|_{L^{2}(\Omega)}^{2}=a(z, \psi)=a(z, \psi-I \psi) \leq C\|z\|_{H^{1}(\Omega)}\|\psi-I \psi\|_{H^{1}(\Omega)} .
$$

From (2.15) in Lemma 2.8, we have $\|\psi\|_{H^{3 / 2+\varepsilon}(\Omega)} \leq C_{\varepsilon}\left\|\delta_{\Gamma}^{-1 / 2+\varepsilon} z\right\|_{L^{2}(\Omega)}$ so that with the approximation properties of $V_{h}$ we get

$$
\inf _{I \psi \in V_{h}}\|\psi-I \psi\|_{H^{1}(\Omega)} \leq C_{\varepsilon} h^{1 / 2+\varepsilon}\left\|\delta_{\Gamma}^{-1 / 2+\varepsilon} z\right\|_{L^{2}(\Omega)} .
$$

This shows (3.2). For (3.3), we proceed similarly using the regularity assertion (2.13) and the approximation property of $V_{h}$.

Corollary 3.2 Let Assumption 1.1 be valid and the solution $u$ be in $H^{s}(\Omega), s \geq 1$. Then the FEM error $e=u-u_{h}$ satisfies for $\varepsilon \in\left(0, s_{0}-1 / 2\right]$

$$
\left\|\delta_{\Gamma}^{-1 / 2+\varepsilon} e\right\|_{L^{2}(\Omega)} \leq C_{\varepsilon} h^{\mu-1 / 2+\varepsilon}\|u\|_{H^{\mu}(\Omega)}, \quad \mu:=\min \{s, k+1\} .
$$

The following Theorem 3.3 shows that the optimal rate of the $L^{2}$-convergence of the FEM can be achieved also for non-convex geometries if the solution has some additional regularity:

Theorem 3.3 Let Assumption 1.1 be valid. Let the exact solution $u$ satisfy the extra regularity $u \in H^{k+2-s_{0}}(\Omega)$. Then the FEM error $u-u_{h}$ satisfies

$$
\left\|u-u_{h}\right\|_{L^{2}(\Omega)} \lesssim h^{k+1}\|u\|_{H^{k+2-s_{0}(\Omega)}} .
$$

More generally, if $u \in H^{s}(\Omega), s \in\left[1, k+2-s_{0}\right]$, then

$$
\left\|u-u_{h}\right\|_{L^{2}(\Omega)} \lesssim h^{s-1+s_{0}}\|u\|_{H^{s}(\Omega)}, \quad 1 \leq s \leq k+2-s_{0} .
$$


Proof of (3.4): We proceed along a standard duality argument. To begin with, we note that the case $s_{0}=1$ is classical so that we may assume $s_{0}<1$ for the remainder of the proof. Set $\varepsilon:=s_{0}-1 / 2 \in(0,1 / 2)$ by our assumption $1 / 2<s_{0}<$ 1. Let $w=T e$ and let $w_{h} \in V_{h}$ be its Galerkin approximation. Quasi-optimality and the use of (2.6) give us the following energy error estimate:

$$
\begin{aligned}
\left\|w-w_{h}\right\|_{H^{1}(\Omega)} & \lesssim \inf _{v \in V_{h}}\|w-v\|_{H^{1}(\Omega)} \lesssim h^{1 / 2+\varepsilon}\|w\|_{H^{3 / 2+\varepsilon}(\Omega)} \\
& \lesssim h^{1 / 2+\varepsilon}\|e\|_{\left(H^{1 / 2-\varepsilon}(\Omega)\right)^{\prime}} \lesssim h^{1 / 2+\varepsilon}\|e\|_{L^{2}(\Omega)} .
\end{aligned}
$$

The Galerkin orthogonalities satisfied by $e$ and $w-w_{h}$ and a weighted CauchySchwarz inequality yield for the Scott-Zhang interpolant $I_{h}^{k} u$

$$
\begin{aligned}
\|e\|_{L^{2}(\Omega)}^{2} & =a(e, w)=a\left(e, w-w_{h}\right)=a\left(u-I_{h}^{k} u, w-w_{h}\right) \\
& \leq C\left\|\widetilde{\delta}_{\Gamma}^{-1 / 2+\varepsilon} \nabla(u-I u)\right\|_{L^{2}(\Omega)}\left\|\widetilde{\delta}_{\Gamma}^{1 / 2-\varepsilon} \nabla\left(w-w_{h}\right)\right\|_{L^{2}(\Omega)} .
\end{aligned}
$$

We get by a covering argument and (2.6) of Lemma 2.1

$$
\begin{aligned}
\left\|\widetilde{\delta}_{\Gamma}^{-1 / 2+\varepsilon} \nabla\left(u-I_{h}^{k} u\right)\right\|_{L^{2}(\Omega)} & \lesssim h^{k}\left\|\widetilde{\delta}_{\Gamma}^{-1 / 2+\varepsilon} \nabla^{k+1} u\right\|_{L^{2}(\Omega)} \\
& \lesssim h^{k}\left\|\nabla^{k+1} u\right\|_{H^{1 / 2-\varepsilon}(\Omega)} .
\end{aligned}
$$

It should also be noted at this point that in (3.9), the weight $\widetilde{\delta}_{\Gamma}^{-1 / 2+\varepsilon}$ can be considered as constant in each element $K$. For the contribution $\| \widetilde{\delta}_{\Gamma}^{1 / 2-\varepsilon} \nabla(w-$ $\left.w_{h}\right) \|_{L^{2}(\Omega)}$ in (3.8), we have to analyze the Galerkin error $w-w_{h}$ in more detail, which will be done with the techniques from the local error analysis of the FEM. We split $\Omega$ into $S_{c h} \cup\left(\Omega \backslash S_{c h}\right)$ where $c>0$ will be selected sufficiently large below. For fixed $c>0$, the $L^{2}$-norm on $S_{c h}$ can easily be bounded with (3.6) by

$$
\left\|\widetilde{\delta}_{\Gamma}^{1 / 2-\varepsilon} \nabla\left(w-w_{h}\right)\right\|_{L^{2}\left(S_{c h}\right)} \lesssim h^{1 / 2-\varepsilon}\left\|\nabla\left(w-w_{h}\right)\right\|_{L^{2}(\Omega)} \lesssim h\|e\|_{L^{2}(\Omega)} .
$$

The term $\left\|\widetilde{\delta}_{\Gamma}^{1 / 2-\varepsilon} \nabla\left(w-w_{h}\right)\right\|_{L^{2}\left(\Omega \backslash S_{c h}\right)}$ requires more care. Obviously, $\widetilde{\delta}_{\Gamma}^{1 / 2-\varepsilon} \lesssim$ $\delta_{\Gamma}^{1 / 2-\varepsilon}$ on $\Omega \backslash S_{c h}$. We have to employ the tools from the local error analysis in FEM. The Galerkin orthogonality satisfied by $w-w_{h}$ allows us to use techniques as described in [24, Sec. 5.3], which yields the following estimate for arbitrary balls $B_{r} \subset B_{r^{\prime}}$ with the same center (implicitly, $r^{\prime}>r+O(h)$ is assumed in (3.11))

$$
\left\|\nabla\left(w-w_{h}\right)\right\|_{L^{2}\left(B_{r}\right)} \lesssim\left\|\nabla\left(w-I_{h}^{k} w\right)\right\|_{L^{2}\left(B_{r^{\prime}}\right)}+\frac{1}{r^{\prime}-r}\left\|w-w_{h}\right\|_{L^{2}\left(B_{r^{\prime}}\right)} .
$$

By a covering argument (which requires $r^{\prime}-r \sim c \delta_{\Gamma}(x)$, where $x$ is the center of the ball $B_{r}$, and $c$ is sufficiently small) these local estimates can be combined into a global estimate of the following form, where for sufficiently small $c_{1}>0\left(c_{1}\right.$ depends only on $\Omega$ and the shape regularity of the triangulation but is independent of $h)$ :

$$
\begin{aligned}
& \left\|\delta_{\Gamma}^{1 / 2-\varepsilon} \nabla\left(w-w_{h}\right)\right\|_{L^{2}\left(\Omega \backslash S_{c h}\right)} \lesssim \\
& \quad\left\|\delta_{\Gamma}^{1 / 2-\varepsilon} \nabla\left(w-I_{h}^{k} w\right)\right\|_{L^{2}\left(\Omega \backslash S_{c c_{1} h}\right)}+\left\|\delta_{\Gamma}^{-1 / 2-\varepsilon}\left(w-w_{h}\right)\right\|_{L^{2}\left(\Omega \backslash S_{c c_{1} h}\right)} .
\end{aligned}
$$

This estimate implicitly assumed $c_{1} c h>2 h$, i.e., at least two layers of elements separate $\Gamma$ from $\Omega \backslash S_{c_{1} c h}$. We now fix $c>2 / c_{1}$. The first term in (3.12) can 
easily be bounded by standard approximation properties of $I_{h}^{k}$, Lemma 2.3, and Assumption 1.1.

$$
\begin{array}{r}
\left\|\delta_{\Gamma}^{1 / 2-\varepsilon} \nabla\left(w-I_{h}^{k} w\right)\right\|_{L^{2}\left(\Omega \backslash S_{c c_{1} h}\right)} \lesssim h\left\|\delta_{\Gamma}^{1 / 2-\varepsilon} \nabla^{2} w\right\|_{L^{2}(\Omega)} \\
\quad \lesssim h\left[\left\|\delta_{\Gamma}^{1 / 2-\varepsilon} e\right\|_{L^{2}(\Omega)}+\|w\|_{H^{3 / 2+\varepsilon}(\Omega)}\right] \lesssim h\|e\|_{L^{2}(\Omega)}
\end{array}
$$

In the last step, we have to deal with the term $\left\|\delta_{\Gamma}^{-1 / 2-\varepsilon}\left(w-w_{h}\right)\right\|_{L^{2}\left(\Omega \backslash S_{c c_{1} h}\right)}$ of (3.12). Lemma 3.1 and (3.6) imply

$$
\begin{aligned}
\left\|\delta_{\Gamma}^{-1 / 2-\varepsilon}\left(w-w_{h}\right)\right\|_{L^{2}\left(\Omega \backslash S_{c c_{1} h}\right)} & \lesssim h^{-2 \varepsilon}\left\|\delta_{\Gamma}^{-1 / 2+\varepsilon}\left(w-w_{h}\right)\right\|_{L^{2}(\Omega)} \\
& \lesssim h^{-2 \varepsilon} h^{1 / 2+\varepsilon}\left\|w-w_{h}\right\|_{H^{1}(\Omega)} \lesssim h\|e\|_{L^{2}(\Omega)} .
\end{aligned}
$$

Here we have used the quasi-optimality of the Galerkin approximation with respect to the $H^{1}$-norm.

Proof of (3.5): The above arguments show that the regularity of $u$ enters in the bound (3.9). For $u \in H^{1}(\Omega)$, the stability properties of the Scott-Zhang operator $I_{h}^{k}$ show

$$
\left\|\widetilde{\delta}_{\Gamma}^{-1 / 2+\varepsilon} \nabla\left(u-I_{h}^{k} u\right)\right\|_{L^{2}(\Omega)} \lesssim h^{-1 / 2+\varepsilon}\|u\|_{H^{1}(\Omega)} .
$$

Hence, a standard interpolation argument that combines (3.9) and (3.14) yields $\left\|\widetilde{\delta}_{\Gamma}^{-1 / 2+\varepsilon} \nabla\left(u-I_{h}^{k} u\right)\right\|_{L^{2}(\Omega)} \lesssim h^{-1 / 2+\varepsilon+s-1}\|u\|_{H^{s}(\Omega)}$ for $s \in\left[1, k+2-s_{0}\right]$. Combining this estimate with the above control of $\left\|\widetilde{\delta}_{\Gamma}^{1 / 2-\varepsilon} \nabla\left(w-w_{h}\right)\right\|_{L^{2}(\Omega)}$ yields the desired bound in the range $s \in\left[1, k+2-s_{0}\right]$.

\section{FEM $L^{2}$-error analysis on piecewise smooth geometries}

The convergence analysis of Theorem 3.3 did not make explicit use of the fact that a piecewise smooth geometry is considered; the essential ingredient was Assumption 1.1 (which, of course, is related to the geometry of the problem). This is reflected in our use of $\widetilde{\delta}_{\Gamma}$, which measures the distance from the boundary $\Gamma$. One interpretation of this procedure is that one assumes of the dual solution $w$ (and, in fact, also of the solution of the "bidual" problem employed to estimate $\left\|\widetilde{\delta}_{\Gamma}^{-1 / 2+\varepsilon}\left(w-w_{h}\right)\right\|_{L^{2}(\Omega)}$ in Theorem 3.3) that it may lose $H^{2}$-regularity anywhere near $\Gamma$. However, for piecewise smooth geometries in conjunction with certain homogeneous boundary conditions (here: homogeneous Dirichlet conditions), this loss of $H^{2}$-regularity is restricted to a much smaller set, namely, a subset of vertices in $2 \mathrm{D}$ and a subset of the skeleton (i.e., the union of vertices and edges) in 3D. This set is given by $M_{s}$ in Definition 2.5. For this set $M_{s}$, we introduce the distance function

$$
\delta_{M_{s}}:=\operatorname{dist}\left(\cdot, M_{s}\right), \quad \widetilde{\delta}_{M_{s}}:=h+\delta_{M_{s}} .
$$

Theorem 4.1 Let $\Omega$ be a polygon (in 2D) or a polyhedron (in 3D). Let $M_{s}$ be the set of vertices (in 2D) or edges and vertices (in 3D) associated with a loss of $H^{2}$-regularity for (1.3) as given in Definition 2.5. Let Assumption 1.1 be valid. Let Iu $\in V_{h}$ be arbitrary. Then we have

$$
\left\|u-u_{h}\right\|_{L^{2}(\Omega)} \leq C h\left\|\widetilde{\delta}_{M_{s}}^{s_{0}-1} \nabla(u-I u)\right\|_{L^{2}(\Omega)} .
$$


Proof We may assume $s_{0}<1$ since the case $s_{0}=1$ corresponds to the standard duality argument with full elliptic regularity and set $\varepsilon:=s_{0}-1 / 2 \in(0,1 / 2)$. The key observation is that, starting from the duality argument (3.7), one can replace the weight function $\widetilde{\delta}_{\Gamma}^{-1 / 2+\varepsilon}$ in (3.8) with any positive weight function. Taking as the weight function $\widetilde{\delta}_{M_{s}}^{-1 / 2+\varepsilon}$, we get

$$
\|e\|_{L^{2}(\Omega)}^{2} \lesssim\left\|\widetilde{\delta}_{M_{s}}^{-1 / 2+\varepsilon} \nabla(u-I u)\right\|_{L^{2}(\Omega)}\left\|\widetilde{\delta}_{M_{s}}^{1 / 2-\varepsilon} \nabla\left(w-w_{h}\right)\right\|_{L^{2}(\Omega)} .
$$

The estimate of $w-w_{h}$ in the weighted norm is done similarly as in the proof of Theorem 3.3. taking into account the improved knowledge of the regularity of $w$. With $S_{M_{s}, c h}:=\left\{x \in \Omega \mid \delta_{M_{s}}(x)<c h\right\}$ we have the trivial bound

$$
\begin{aligned}
& \left\|\widetilde{\delta}_{M_{s} / 2-\varepsilon}^{1 / 2} \nabla\left(w-w_{h}\right)\right\|_{L^{2}(\Omega)} \\
& \quad \lesssim\left\|\widetilde{\delta}_{M_{s} / 2-\varepsilon}^{1 / 2} \nabla\left(w-w_{h}\right)\right\|_{L^{2}\left(S_{M_{s}, c h}\right)}+\left\|\widetilde{\delta}_{M_{s}}^{1 / 2-\varepsilon} \nabla\left(w-w_{h}\right)\right\|_{L^{2}\left(\Omega \backslash S_{M_{s}, c h}\right)},
\end{aligned}
$$

where the parameter $c$ will be selected sufficiently large below. The first term in (4.3) is estimated in exactly the same way as in (3.10) and produces

$$
\left\|\widetilde{\delta}_{M_{s}}^{1 / 2-\varepsilon} \nabla\left(w-w_{h}\right)\right\|_{L^{2}\left(S_{M_{s}, c h}\right)} \leq C h\|e\|_{L^{2}(\Omega)} .
$$

The second term in (4.2) again requires the techniques from the local error analysis of the FEM, this time with the appropriate modifications to account for the boundary conditions. Inspection of the arguments in [24, Sec. 5.3] shows that the key estimate (3.11) extends up to the boundary in the following sense:

$$
\left\|\nabla\left(w-w_{h}\right)\right\|_{L^{2}\left(B_{r} \cap \Omega\right)} \lesssim\left\|\nabla\left(w-I_{h}^{k} w\right)\right\|_{L^{2}\left(B_{r^{\prime}} \cap \Omega\right)}+\frac{1}{r^{\prime}-r}\left\|w-w_{h}\right\|_{L^{2}\left(B_{r^{\prime}} \cap \Omega\right)} ;
$$

besides the implicit assumption $r^{\prime}>r+O(h)$, the balls $B_{r}$ and $B_{r^{\prime}}$ are assumed to have the same center $x$ and satisfy one of the following conditions:

1. $B_{r^{\prime}}=B_{r^{\prime}}(x) \subset \Omega$;

2. $x \in \partial \Omega$ and $B_{r^{\prime}}(x) \cap \Omega$ is a half-disk;

3. $x$ is a vertex of $\Omega$;

4. (only for $d=3$ ) $x$ lies on an edge $e$ and $B_{r^{\prime}}(x) \cap \Omega$ is a dihedral angle (i.e., the intersection of $\partial\left(B_{r^{\prime}}(x) \cap \Omega\right)$ with $\partial \Omega$ is contained in the two faces that share the edge $e$.

The reason for the restriction of the location of the centers of the balls is that the procedure presented in [24, Sec. 5.3] relies on Poincaré inequalities so that the number of possible shapes for the intersections $B_{r^{\prime}} \cap \Omega$ should be finite. A covering argument (see Theorem A.5 for the 2D case and Theorem A.6 for the $3 \mathrm{D}$ situation) then leads to the following bound with an appropriate $c_{1}>0$ (here, $c>0$ is implicitly assumed sufficiently large):

$$
\begin{aligned}
& \left\|\widetilde{\delta}_{M_{s}}^{1 / 2-\varepsilon} \nabla\left(w-w_{h}\right)\right\|_{L^{2}\left(\Omega \backslash S_{M_{s}, c h}\right)} \lesssim \\
& \quad\left\|\widetilde{\delta}_{M_{s} / 2-\varepsilon}^{1 / 2} \nabla\left(w-I_{h}^{k} w\right)\right\|_{L^{2}\left(\Omega \backslash S_{M_{s}, c c_{1} h}\right)}+\left\|\widetilde{\delta}_{M_{s}}^{-1 / 2-\varepsilon}\left(w-w_{h}\right)\right\|_{L^{2}\left(\Omega \backslash S_{M_{s}, c c_{1} h}\right)} .
\end{aligned}
$$


The first term in (4.5) can be estimated with the improved regularity assertion of Lemma 2.6 to produce (with appropriate $c_{2}>0$ and the implicit assumption on $c$ that $c c_{1} c_{2}>2$ )

$$
\begin{aligned}
& \left\|\widetilde{\delta}_{M_{s}}^{1 / 2-\varepsilon} \nabla\left(w-I_{h}^{k} w\right)\right\|_{L^{2}\left(\Omega \backslash S_{M_{s}, c c_{1} h}\right)} \lesssim h\left\|\widetilde{\delta}_{M_{s}}^{1 / 2-\varepsilon} \nabla^{2} w\right\|_{L^{2}\left(\Omega \backslash S_{M_{s}, c c_{1} c_{2} h}\right)} \\
& \quad \lesssim h\left[\left\|\widetilde{\delta}_{M_{s}}^{1 / 2-\varepsilon} e\right\|_{L^{2}(\Omega)}+\|w\|_{H^{3 / 2+\varepsilon}(\Omega)}\right] \lesssim h\|e\|_{L^{2}(\Omega)} .
\end{aligned}
$$

For the second term in (4.5) we note that $-1 / 2-\varepsilon<0$ so that $\widetilde{\delta}_{M_{s}}^{-1 / 2-\varepsilon} \leq \widetilde{\delta}_{\Gamma}^{-1 / 2-\varepsilon}$. This leads to

$$
\begin{aligned}
& \left\|\widetilde{\delta}_{M_{s}}^{-1 / 2-\varepsilon}\left(w-w_{h}\right)\right\|_{L^{2}\left(\Omega \backslash S_{M_{s}, c c_{1} h}\right)} \lesssim\left\|\widetilde{\delta}_{M_{s}}^{-1 / 2-\varepsilon}\left(w-w_{h}\right)\right\|_{L^{2}(\Omega)} \\
& \quad \lesssim\left\|\widetilde{\delta}_{\Gamma}^{-1 / 2-\varepsilon}\left(w-w_{h}\right)\right\|_{L^{2}(\Omega)} \lesssim h^{-2 \varepsilon}\left\|\widetilde{\delta}_{\Gamma}^{-1 / 2+\varepsilon}\left(w-w_{h}\right)\right\|_{L^{2}(\Omega)}
\end{aligned}
$$

the term $h^{-2 \varepsilon}\left\|\widetilde{\delta}_{\Gamma}^{-1 / 2+\varepsilon}\left(w-w_{h}\right)\right\|_{L^{2}(\Omega)}$ has already been estimated in (3.13) in the desired form.

The regularity requirements on the solution $u$ can still be slightly weakened. As written, the exponent $s_{0}-1$ is related to the global regularity of the dual solution $w$. However, the developments above show that a local lack of full regularity of the dual solution $w$ (and the bidual solution) needs to be offset by additional local regularity of the solution. To be more specific, we restrict our attention now to the $2 \mathrm{D}$ Laplacian, i.e., $\mathbf{A}=\mathrm{Id}$. In this case, the situation can be expressed as follows with the aid of the singular exponents $\alpha_{j}:=\pi / \omega_{j}$, where $\omega_{j} \in(\pi, 2 \pi)$ is the interior angle at the reentrant vertices $A_{j}, j=1, \ldots, J$.

Corollary 4.2 Let $\Omega \subset \mathbb{R}^{2}$ be a polygon and let $\mathbf{A}=$ Id. Let $\delta_{j}:=\operatorname{dist}\left(\cdot, A_{j}\right)$, $j=1, \ldots, J$, for the $J$ reentrant corners. Set $\widetilde{\delta}_{j}:=h+\delta_{j}$. Let $\omega_{j}$ be the interior angle at $A_{j}$ and $\alpha_{j}=\pi / \omega_{j}$. Fix $\beta_{j}>1-\alpha_{j}$ arbitrary. Then for any $I u \in V_{h}$

$$
\left\|u-u_{h}\right\|_{L^{2}(\Omega)} \lesssim h \sum_{j=1}^{J}\left\|\widetilde{\delta}_{j}^{-\beta_{j}} \nabla(u-I u)\right\|_{L^{2}(\Omega)} .
$$

Proof The proof follows by an inspection of how the regularity of the solution $w=T e$ of the dual problem enters the proof of Theorem 4.1. By, e.g., [10 the solution $w=T e$ is in a weighted $H^{2}$-space with

$$
\left\|\prod_{j=1}^{J} \delta_{j}^{\beta_{j}} \nabla^{2} w\right\|_{L^{2}(\Omega)} \lesssim\|e\|_{L^{2}(\Omega)},
$$

and Assumption 1.1 holds with any $s_{0}<\min _{j} \alpha_{j}$. The regularity assertion (4.6) suggests to choose $\prod_{i=1}^{J} \widetilde{\delta}_{i}^{\beta_{i}}$ as the weight in the proof of Theorem 3.3. Inspection of the procedure in the proof of Theorem 4.1 then leads to the result.

We extract from this result another corollary that we will prove useful in the numerical results. We formulate it in terms of (standard, unweighted) Sobolev regularity in order to emphasize the difference in regularity requirements of the solution near the reentrant corners and away from them: 
Corollary 4.3 Assume the hypotheses of Corollary 4.2. Let $s>1$ and $s_{i}>1$, $i=1, \ldots, J$. Let $\mathcal{U}:=\Omega \backslash \cup \overline{\mathcal{U}}_{i}$, for some neighborhoods $\mathcal{U}_{i}$ of the reentrant vertices $A_{i}$. Let $u \in H^{s_{i}}\left(\mathcal{U}_{i}\right), i=1, \ldots, J$ and $u \in H^{s}(\mathcal{U})$. Then for arbitrary $\varepsilon>0$

$$
\left\|u-u_{h}\right\|_{L^{2}(\Omega)} \leq C_{\varepsilon} h^{\tau}, \quad \tau:=\min \left(1+k, s, \min _{j=1, \ldots, J}\left(-1+\alpha_{j}+s_{j}-\varepsilon\right)\right) .
$$

Proof The approximant $I u$ in Corollary 4.2 may be taken as any standard nodal interpolant or the Scott-Zhang projection. Then standard estimates and Corollary 4.2 produce with the choice $\beta_{j}:=1-\alpha_{j}+\varepsilon$ for arbitrary small but fixed $\varepsilon>0$ :

$$
\begin{aligned}
\left\|u-u_{h}\right\|_{L^{2}(\Omega)} & \lesssim h \min _{j=1, \ldots, J}\left\{h^{\min \{k, s-1\}}, h^{-\beta_{j}+s_{j}-1}\right\} \\
& \lesssim \min _{j=1, \ldots, J}\left\{h^{\min \{k+1, s\}}, h^{\alpha_{j}+s_{j}-1-\varepsilon}\right\} .
\end{aligned}
$$

\section{Optimal $L^{2}\left(S_{h}\right)$-convergence}

Additional regularity of the solution also allows us to prove that the error on the strip $S_{h}$ of width $O(h)$ near $\Gamma$ is of higher order:

Theorem 5.1 Let Assumption 1.1 be valid. Then the FEM error $u-u_{h}$ satisfies

$$
\begin{aligned}
&\left\|u-u_{h}\right\|_{L^{2}\left(S_{h}\right)} \lesssim h^{k+3 / 2}\left(1+\delta_{k, 1}|\ln h|\right)\|u\|_{B_{2,1}^{k+3 / 2}(\Omega)}, \\
&\left\|u-u_{h}\right\|_{L^{2}\left(S_{h}\right)} \lesssim h^{s+3 / 2}\left(1+\delta_{k, 1}|\ln h|\right)\|u\|_{B_{2, \infty}^{s+3 / 2}(\Omega)}, \quad s \in(0, k), \\
&\left\|u-u_{h}\right\|_{L^{2}\left(S_{h}\right)} \lesssim h^{3 / 2}\left(1+\delta_{k, 1}|\ln h|\right)\|u\|_{B_{2,1}^{3 / 2}(\Omega)},
\end{aligned}
$$

where $\delta_{k, 1}$ is the Kronecker symbol. The implies constant depends on the shape regularity of the triangulation, $\Omega$, and the coefficient $\mathbf{A}$. Specifically, it depends on $\alpha_{0}$ and $\|\mathbf{A}\|_{C^{0,1}(\bar{\Omega})}$ and, in the case $k>1$, additionally on $\|\mathbf{A}\|_{C^{1,1}(\bar{\Omega})}$.

Remark 5.2 1. The regularity requirement $B_{2,1}^{k+3 / 2}(\Omega)$ can be weakened: it suffices that $u$ be in $B_{2,1}^{k+3 / 2}\left(S_{D}\right)$ in a fixed neighborhood $S_{D}$ of $\Gamma$ and in $H^{k+1}(\Omega)$. See [16] for the details of a closely related problem.

2. Since $B_{2, \infty}^{s+3 / 2}(\Omega) \supset H^{s+3 / 2}(\Omega)$, the assertions for $s \in(0, k)$ can be weakened by replacing $\|u\|_{B_{2, \infty}^{s+3 / 2}(\Omega)}$ with $\|u\|_{H^{s+3 / 2}(\Omega)}$ on the right-hand side. Only for the limiting cases $s=0$ and $s=k$, we require the stronger requirement $u \in$ $B_{2,1}^{s+3 / 2}(\Omega) \subset H^{s+3 / 2}(\Omega)$.

Proof The structure of the proof is very similar to that of Theorem 3.3. The main difference arises from the fact that the right-hand side of the dual problem is supported by the thin neighborhood $S_{h}$, and this support property has to be exploited. 
Let $e=u-u_{h}$. Let $\chi_{S_{h}}$ be the characteristic function of $S_{h}$. Let $w=T\left(\chi_{S_{h}} e\right)$ and $w_{h} \in V_{h}$ its Galerkin approximation. Again, Galerkin orthogonality for $u-u_{h}$ and $w-w_{h}$ implies

$$
\begin{aligned}
\|e\|_{L^{2}\left(S_{h}\right)}^{2} & =\langle e, \chi e\rangle=a(e, w)=a\left(e, w-w_{h}\right)=a\left(u-I_{h}^{k} u, w-w_{h}\right) \\
& \leq C\left\|\widetilde{\delta}_{\Gamma}^{-1 / 2-\varepsilon} \nabla\left(u-I_{h}^{k} u\right)\right\|_{L^{2}(\Omega)}\left\|\widetilde{\delta}_{\Gamma}^{1 / 2+\varepsilon} \nabla\left(w-w_{h}\right)\right\|_{L^{2}(\Omega)},
\end{aligned}
$$

where $\varepsilon \geq 0$ is arbitrary (in fact, $\varepsilon \in \mathbb{R}$ would be admissible). We flag at this point already that we will select $\varepsilon=0$ for $k=1$ and $\varepsilon>0$ arbitrary (but sufficiently small) for $k>1$. Each of the two factors in (5.1) is estimated separately.

1. step: For the first factor in (5.1) we use approximation properties of the Scott-Zhang operator $I_{h}^{k}$ together with Lemma 2.1 to get for $j \in\{0, \ldots, k\}$

$$
\begin{aligned}
\left\|\widetilde{\delta}_{\Gamma}^{-1 / 2-\varepsilon} \nabla\left(u-I_{h}^{k} u\right)\right\|_{L^{2}(\Omega)} & \lesssim h^{j}\left\|\widetilde{\delta}_{\Gamma}^{-1 / 2-\varepsilon} \nabla^{j+1} u\right\|_{L^{2}(\Omega)}, \\
& \lesssim h^{j} \begin{cases}|\ln h|^{1 / 2}\left\|\nabla^{j+1} u\right\|_{B_{2,1}^{1 / 2}(\Omega)} & \text { if } \varepsilon=0 \\
h^{-\varepsilon}\left\|\nabla^{j+1} u\right\|_{B_{2,1}^{1 / 2}(\Omega)} & \text { if } \varepsilon>0 .\end{cases}
\end{aligned}
$$

With the Kronecker symbol $\delta_{0, \varepsilon}$, we have shown for $j \in\{0,1, \ldots, k\}$

$$
\left\|\widetilde{\delta}_{\Gamma}^{-1 / 2-\varepsilon} \nabla\left(u-I_{h}^{k} u\right)\right\|_{L^{2}(\Omega)} \lesssim h^{j} h^{-\varepsilon}\left(1+\delta_{0, \varepsilon}|\ln h|^{1 / 2}\right)\|u\|_{B_{2,1}^{j+3 / 2}(\Omega)} .
$$

Since the Scott-Zhang operator $I_{h}^{k}$ is defined on $H^{1}(\Omega)$ irrespective of boundary conditions, we may use an interpolation argument to lift the restriction to integer values $j$. Specifically, the reiteration theorem (cf., e.g., [21, Thm. 23.6]) asserts that the Besov space $B_{2, \infty}^{s+3 / 2}(\Omega)$, which we have defined by interpolation between (integer order) Sobolev spaces, coincides with the interpolation space between Besov spaces, viz.,

$$
B_{2, \infty}^{s+3 / 2}(\Omega)=\left(B_{2,1}^{3 / 2}(\Omega), B_{2,1}^{k+3 / 2}(\Omega)\right)_{s / k, \infty} \quad \text { (equivalent norms). }
$$

Hence, we may decompose for arbitrary $t>0$ a function $u \in B_{2, \infty}^{s+3 / 2}(\Omega), s \in(0, k)$, as $u=u-u_{1}+u_{1}$ with $u_{1} \in B_{2,1}^{k+3 / 2}(\Omega)$ and $u_{0}:=u-u_{1} \in B_{2,1}^{3 / 2}(\Omega)$ together with

$$
\left\|u_{0}\right\|_{B_{2,1}^{3 / 2}(\Omega)} \leq C t^{s / k}\|u\|_{B_{2, \infty}^{s+3 / 2}(\Omega)}, \quad\left\|u_{1}\right\|_{B_{2,1}^{k+3 / 2}(\Omega)} \leq C t^{s / k-1}\|u\|_{B_{2, \infty}^{s+3 / 2}(\Omega)} .
$$

Writing $u-I_{h}^{k} u=\left(u_{0}-I_{h}^{k} u_{0}\right)+\left(u_{1}-I_{h}^{k} u_{1}\right)$ we can use (5.3) with $j=k$ for the second term in brackets and $j=0$ for the first term in brackets to get with the choice $t=h^{k}$

$$
\left\|\widetilde{\delta}_{\Gamma}^{-1 / 2-\varepsilon} \nabla\left(u-I_{h}^{k} u\right)\right\|_{L^{2}(\Omega)} \lesssim h^{s} h^{-\varepsilon}\left(1+\delta_{0, \varepsilon}|\ln h|^{1 / 2}\right)\|u\|_{B_{2, \infty}^{s+3 / 2}(\Omega)} .
$$

Combining the estimates (5.3) with $j=0$ and $j=k$ and (5.4) for $s \in(0, k)$ we arrive at

$$
\begin{aligned}
& \left\|\widetilde{\delta}_{\Gamma}^{-1 / 2-\varepsilon} \nabla\left(u-I_{h}^{k} u\right)\right\|_{L^{2}(\Omega)} \\
& \lesssim\left(1+\delta_{0, \varepsilon}|\ln h|^{1 / 2}\right) h^{-\varepsilon} \begin{cases}h^{s}\|u\|_{B_{2,1}^{s+3 / 2}(\Omega)}, & s=0, \\
h^{s}\|u\|_{B_{2, \infty}^{s+3 / 2}(\Omega)}, & s \in(0, k), \\
h^{s}\|u\|_{B_{2,1}^{k+3 / 2}(\Omega)}, & s=k .\end{cases}
\end{aligned}
$$


2. step: The second factor in (5.1) requires more work. We start with a regularity assertion for $w$ that exploits the support properties of $\chi_{S_{h}} e$ and follows from (2.11) and (2.12):

$$
\begin{aligned}
\|w\|_{B_{2, \infty}^{3 / 2}(\Omega)} & \lesssim h^{1 / 2}\left\|\chi_{S_{h}} e\right\|_{L^{2}(\Omega)}, \\
\|w\|_{H^{3 / 2+\varepsilon}(\Omega)} & \lesssim h^{1 / 2-\varepsilon}\left\|\chi_{S_{h}} e\right\|_{L^{2}(\Omega)}, \quad \varepsilon \in\left(0, s_{0}-1 / 2\right] .
\end{aligned}
$$

We obtain an energy error estimate for $w-w_{h}$ in the standard way by using quasi-optimality, the approximation properties of $V_{h}$, and the regularity assertion (5.6):

$$
\left\|w-w_{h}\right\|_{H^{1}(\Omega)} \lesssim \inf _{v \in V_{h}}\|w-v\|_{H^{1}(\Omega)} \lesssim h^{1 / 2}\|w\|_{B_{2, \infty}^{3 / 2}(\Omega)} \lesssim h\left\|\chi_{S_{h}} e\right\|_{L^{2}(\Omega)} .
$$

Lemma 3.1 is applicable with $z=w-w_{h}$; hence, obtain with (5.8)

$$
\begin{aligned}
\left\|\delta_{\Gamma}^{-1 / 2+\varepsilon}\left(w-w_{h}\right)\right\|_{L^{2}(\Omega)} & \lesssim h^{3 / 2+\varepsilon}\left\|\chi_{S_{h}} e\right\|_{L^{2}(\Omega)}, \quad \varepsilon \in\left(0, s_{0}-1 / 2\right] \\
\left\|\widetilde{\delta}_{\Gamma}^{-1 / 2}\left(w-w_{h}\right)\right\|_{L^{2}(\Omega)} & \lesssim h^{3 / 2}|\ln h|^{1 / 2}\left\|\chi_{S_{h}} e\right\|_{L^{2}(\Omega)}
\end{aligned}
$$

The bound (5.1) informs us that control of $w-w_{h}$ in a weighted $H^{1}$-norm is required. In this direction, we first write for a constant $c>0$ that will be determined later sufficiently large

$$
\begin{aligned}
\left\|\widetilde{\delta}_{\Gamma}^{1 / 2+\varepsilon} \nabla\left(w-w_{h}\right)\right\|_{L^{2}(\Omega)} & \\
& \leq\left\|\widetilde{\delta}_{\Gamma}^{1 / 2+\varepsilon} \nabla\left(w-w_{h}\right)\right\|_{L^{2}\left(S_{c h}\right)}+\left\|\widetilde{\delta}_{\Gamma}^{1 / 2+\varepsilon} \nabla\left(w-w_{h}\right)\right\|_{L^{2}\left(\Omega \backslash S_{c h}\right)} \\
& \leq C h^{1 / 2+\varepsilon}\left\|\nabla\left(w-w_{h}\right)\right\|_{L^{2}(\Omega)}+\left\|\widetilde{\delta}_{\Gamma}^{1 / 2+\varepsilon} \nabla\left(w-w_{h}\right)\right\|_{L^{2}\left(\Omega \backslash S_{c h}\right)} \\
& \quad \leq \quad C h^{3 / 2+\varepsilon}\left\|\chi_{S_{h}} e\right\|_{L^{2}(\Omega)}+\left\|\widetilde{\delta}_{\Gamma}^{1 / 2+\varepsilon} \nabla\left(w-w_{h}\right)\right\|_{L^{2}\left(\Omega \backslash S_{c h}\right)} .
\end{aligned}
$$

We emphasize that $\varepsilon=0$ is allowed in (5.11). It remains to control $\| \widetilde{\delta}_{\Gamma}^{1 / 2+\varepsilon} \nabla(w-$ $\left.w_{h}\right) \|_{L^{2}\left(\Omega \backslash S_{c h}\right)}$. This is done again with the same arguments from the local error analysis as in the proof of Theorem 3.3. The estimate (3.12) holds verbatim, i.e.,

$$
\begin{aligned}
& \left\|\delta_{\Gamma}^{1 / 2+\varepsilon} \nabla\left(w-w_{h}\right)\right\|_{L^{2}\left(\Omega \backslash S_{c h}\right)} \\
& \quad \lesssim\left\|\delta_{\Gamma}^{1 / 2+\varepsilon} \nabla\left(w-I_{h}^{k} w\right)\right\|_{L^{2}\left(\Omega \backslash S_{c c_{1} h}\right)}+\left\|\delta_{\Gamma}^{-1 / 2+\varepsilon}\left(w-w_{h}\right)\right\|_{L^{2}\left(\Omega \backslash S_{c c_{1} h}\right)} .
\end{aligned}
$$

We emphasize that $\varepsilon=0$ is admissible in (3.12). As in the proof of Theorem 3.3 . the constant $c$ will be selected in dependence of various inverse estimates that are applied. Combining (5.9), (5.10), (5.11), (5.12) we see that we have shown

$$
\begin{aligned}
& \left\|\widetilde{\delta}_{\Gamma}^{1 / 2+\varepsilon} \nabla\left(w-w_{h}\right)\right\|_{L^{2}(\Omega)} \\
& \quad \lesssim \begin{cases}h^{3 / 2+\varepsilon}\left\|\chi_{S_{h}} e\right\|_{L^{2}(\Omega)}+\left\|\widetilde{\delta}_{\Gamma}^{1 / 2+\varepsilon} \nabla\left(w-I_{h}^{k} w\right)\right\|_{L^{2}\left(\Omega \backslash S_{c_{1} c h}\right)} & \text { if } \varepsilon>0, \\
h^{3 / 2}|\ln h|^{1 / 2}\left\|\chi_{S_{h}} e\right\|_{L^{2}(\Omega)}+\left\|\widetilde{\delta}_{\Gamma}^{1 / 2} \nabla\left(w-I_{h}^{k} w\right)\right\|_{L^{2}\left(\Omega \backslash S_{c_{1} c h}\right)} & \text { if } \varepsilon=0 .\end{cases}
\end{aligned}
$$

3. step: We estimate the approximation error $\left\|\widetilde{\delta}_{\Gamma}^{1 / 2+\varepsilon} \nabla\left(w-I_{h}^{k} w\right)\right\|_{L^{2}\left(\Omega \backslash S_{c_{1} c h}\right)}$. At this point the cases $k=1$ and $k>1$ diverge: since $w$ solves a homogeneous elliptic equation on $\Omega \backslash S_{c_{1} c h}$ (if $c_{1} c>1$ ), interior regularity is available so that higher 
order approximation can be brought to bear if $k>1$ in contrast to the case $k=1$. We start with the simpler case $k=1$.

The case $k=1$ : From standard approximation results for $I_{h}^{k}$, the inverse estimate of Lemma 2.9. (1i), and (5.6) we get for a constant $c_{2} \in(0,1)$ (implicitly, we assume that $c$ is so large that $c_{2} c_{1} c h>2 h$ )

$$
\begin{aligned}
\left\|\widetilde{\delta}_{\Gamma}^{1 / 2} \nabla\left(w-I_{h}^{k} w\right)\right\|_{L^{2}\left(\Omega \backslash S_{c_{1} c h}\right)} & \lesssim h^{1}\left\|\widetilde{\delta}_{\Gamma}^{1 / 2} \nabla^{2} w\right\|_{L^{2}\left(\Omega \backslash S_{c_{2} c_{1} c h}\right)} \\
& \lesssim h|\ln h|^{1 / 2}\|w\|_{B_{2, \infty}^{3 / 2}(\Omega)} \lesssim h^{3 / 2}|\ln h|^{1 / 2}\left\|\chi_{S_{h}} e\right\|_{L^{2}(\Omega)} .
\end{aligned}
$$

Inserting (5.5) (with $\varepsilon=0$ ) with the combination of (5.14) and (5.13) (again with $\varepsilon=0$ ) in (5.1) yields the desired final estimate for the case $k=1$ if we fix $c=2 /\left(c_{1} c_{2}\right)$.

The case $k>1$ : We fix an $\varepsilon \in\left(0, s_{0}-1 / 2\right]$ arbitrary. From standard approximation results for $I_{h}^{k}$, the inverse estimates of Lemma 2.9, and the regularity assertion (5.7) we get (again for suitable constants $c_{2}, c_{3} \in(0,1)$ and the implicit assumption that $c$ is such that $c_{3} c_{2} c_{1} c$ is sufficiently large)

$$
\begin{aligned}
& \left\|\widetilde{\delta}_{\Gamma}^{1 / 2+\varepsilon} \nabla\left(w-I_{h}^{k} w\right)\right\|_{L^{2}\left(\Omega \backslash S_{c_{1} c h}\right)} \lesssim h^{2}\left\|\widetilde{\delta}_{\Gamma}^{1 / 2+\varepsilon} \nabla^{3} w\right\|_{L^{2}\left(\Omega \backslash S_{c_{2} c_{1} c h}\right)} \\
& \text { Lem. } \underset{2.9}{\lesssim} h^{2}\left[\left\|\widetilde{\delta}_{\Gamma}^{-1 / 2+\varepsilon} \nabla^{2} w\right\|_{L^{2}\left(\Omega \backslash S_{c_{3} c_{2} c_{1} c h}\right)}+\left\|\widetilde{\delta}_{\Gamma}^{1 / 2+\varepsilon} \nabla w\right\|_{L^{2}\left(\Omega \backslash S_{c_{3} c_{2} c_{1} c h}\right)}\right] \\
& \lesssim \quad h^{2-1 / 2+\varepsilon}\left[\left\|\nabla^{2} w\right\|_{L^{2}\left(\Omega \backslash S_{c_{3} c_{2} c_{1} c h}\right)}+\|\nabla w\|_{L^{2}\left(\Omega \backslash S_{c_{3} c_{2} c_{1} c h}\right)}\right] \\
& \text { Lem. } \underset{2.9}{\lesssim} h^{2-1 / 2+\varepsilon}\left\|\chi_{S_{h}} e\right\|_{L^{2}(\Omega)} \text {. }
\end{aligned}
$$

Combining this with (5.5) produces in (5.1) the desired final estimate for the case $k>1$.

From Theorem 5.1 we can extract optimal convergence estimates for the flux error $\left\|\partial_{n}\left(u-u_{h}\right)\right\|_{L^{2}(\Gamma)}$ :

Corollary 5.3 Let Assumption 1.1 be valid. Then with the Kronecker symbol $\delta_{k, 1}$

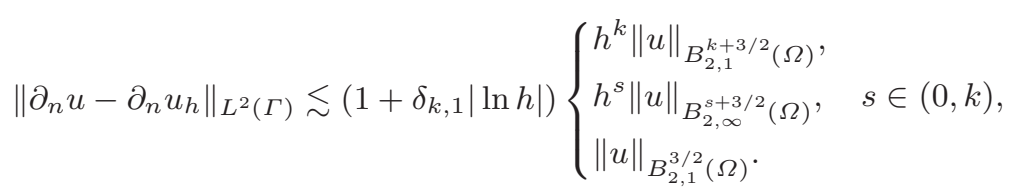

Proof Structurally, the proof follows [18, Cor. 6.1] in that estimating the error on $\Gamma$ is transferred to an estimate on the strip $S_{h}$. The triangle inequality gives

$$
\left\|\partial_{n}\left(u-u_{h}\right)\right\|_{L^{2}(\Gamma)} \leq\left\|\partial_{n}\left(u-I_{h}^{k} u\right)\right\|_{L^{2}(\Gamma)}+\left\|\partial_{n}\left(I_{h}^{k} u-u_{h}\right)\right\|_{L^{2}(\Gamma)} .
$$

The two terms in (5.16) are estimated separately.

1. step: We claim that

$$
\left\|\partial_{n}\left(u-I_{h}^{k} u\right)\right\|_{L^{2}(\Gamma)} \lesssim\left\{\begin{array}{l}
h^{k}\|u\|_{B_{2,1}^{k+3 / 2}(\Omega)} \\
h^{s}\|u\|_{B_{2, \infty}^{s+2}(\Omega)}, \quad s \in(0, k), \\
\|u\|_{B_{2,1}^{3 / 2}(\Omega)}
\end{array}\right.
$$


We will only show the limiting cases $u \in B_{2,1}^{k+3 / 2}(\Omega)$ and $u \in B_{2,1}^{3 / 2}(\Omega)$; the intermediate cases follow by an interpolation argument similar to the one used in the proof of Theorem [5.1. For the case of maximal regularity, we use an elementwise multiplicative trace inequality for the elements abutting $\Gamma$ to get

$$
\begin{gathered}
\left\|\partial_{n}\left(u-I_{h}^{k} u\right)\right\|_{L^{2}(\Gamma)} \lesssim h^{k / 2} \sqrt{\left\|\nabla^{k+1} u\right\|_{L^{2}\left(S_{2 h}\right)}} h^{(k-1) / 2} \sqrt{\left\|\nabla^{k+1} u\right\|_{L^{2}\left(S_{2 h}\right)}} \\
\lesssim h^{k-1 / 2}\left\|\nabla^{k+1} u\right\|_{L^{2}\left(S_{2 h}\right)} \stackrel{\underline{2.4}}{\lesssim} h^{k}\left\|\nabla^{k+1} u\right\|_{B_{2,1}^{1 / 2}(\Omega)} \lesssim h^{k}\|u\|_{B_{2,1}^{k+3 / 2}(\Omega)} .
\end{gathered}
$$

For the case of minimal regularity, $u \in B_{2,1}^{3 / 2}(\Omega)$ we first note that we obtain from (2.5) that $\|v\|_{L^{2}(\Gamma)} \lesssim\|v\|_{B_{2,1}^{1 / 2}(\Omega)}$. Using this and inverse estimates, we get

$$
\begin{aligned}
& \left\|\partial_{n}\left(u-I_{h}^{k} u\right)\right\|_{L^{2}(\Gamma)} \leq\left\|\partial_{n} u\right\|_{L^{2}(\Gamma)}+\left\|\partial_{n} I_{h}^{k} u\right\|_{L^{2}(\Gamma)} \\
& \lesssim\|\nabla u\|_{B_{2,1}^{1 / 2}(\Omega)}+h^{-1 / 2}\left\|\nabla I_{h}^{k} u\right\|_{L^{2}\left(S_{h}\right)} \\
& \stackrel{I_{h}^{k} \text { stable }}{\lesssim}\|\nabla u\|_{B_{2,1}^{1 / 2}(\Omega)}+h^{-1 / 2}\|\nabla u\|_{L^{2}\left(S_{2 h}\right)} \stackrel{\text { 2.4 }}{\lesssim}\|\nabla u\|_{B_{2,1}^{1 / 2}(\Omega)} \text {. }
\end{aligned}
$$

2. step: The term $\left\|\partial_{n}\left(I_{h}^{k} u-u_{h}\right)\right\|_{L^{2}(\Gamma)}$ in (5.16) is controlled with inverse estimates and Theorem 5.1 as follows:

$$
\begin{aligned}
\left\|\partial_{n}\left(I_{h}^{k} u-u_{h}\right)\right\|_{L^{2}(\Gamma)} & \lesssim h^{-1 / 2}\left\|\nabla\left(I_{h}^{k} u-u_{h}\right)\right\|_{L^{2}\left(S_{h}\right)} \lesssim h^{-3 / 2}\left\|I_{h}^{k} u-u_{h}\right\|_{L^{2}\left(S_{h}\right)} \\
& \lesssim h^{-3 / 2}\left\|u-I_{h}^{k} u\right\|_{L^{2}\left(S_{h}\right)}+h^{-3 / 2}\left\|u-u_{h}\right\|_{L^{2}\left(S_{h}\right)} .
\end{aligned}
$$

The term $\left\|u-I_{h}^{k} u\right\|_{L^{2}\left(S_{h}\right)}$ can be controlled with the approximation properties of $I_{h}^{k}$ in the desired fashion: $\left\|u-I_{h}^{k} u\right\|_{L^{2}\left(S_{h}\right)} \lesssim h\|\nabla u\|_{L^{2}\left(S_{2 h}\right)} \lesssim h^{3 / 2}\|\nabla u\|_{B_{2,1}^{1 / 2}(\Omega)}$. The contribution $\left\|u-u_{h}\right\|_{L^{2}\left(S_{h}\right)}$ is estimated with the aid of Theorem 5.1

\section{Extension of the results of [18]}

The arguments of the present paper are similar to those underlying [18, in spite of the fact that we did not employ the anisotropic norms that we introduced in [18] but instead worked with weighted Sobolev spaces. A feature of the analysis here that was not present in [18] is our FEM error analysis in Lemma 3.1] for a weighted $L^{2}$-estimate, which, in turn, relies on the regularity assertions of Lemma 2.8 for problems with data in weighted spaces. This additional technical issue was circumvented in 18 by assuming convexity of $\Omega$ so that optimal order $L^{2}$-estimates could be cited from the literature. The present analysis provides the necessary technical tools to remove this simplification in [18, where a more complex mortar setting is analyzed. It is possible to make use of weighted $L^{2}$-estimates similar to those of Lemma 3.1 in the setting of [18]. For that, regularity results of the type provided in Lemma 2.8 have to be used. The outcome of this refinement is that the main results of [18, namely, [18, Thm. 2.1], which provides $L^{2}$-estimates on strips of width $O(h)$ around the skeleton, and [18, Thm. 2.5], which provides optimal order approximations for the mortar variable, hold true if the geometry is such that Assumption 1.1 is valid. We will not provide the details of the arguments here and refer to [11, Appendix B] instead. Nevertheless, for future reference we record the end result: 
Theorem 6.1 In [18, Thms. 2.1, 2.5], the assumption of convexity of $\Omega$ can be replaced with [18, Assumption (5.2)].

\section{Numerical results}

We consider the simple model equation $-\Delta u=f$ in $\Omega \subset \mathbb{R}, d \in\{2,3\}$ with inhomogeneous Dirichlet boundary conditions. These are realized numerically by nodal interpolation of the prescribed exact solution $u$, and the data $f$ is also computed from $u$. In the case of a non-smooth solution, we use a suitable quadrature formula on finer meshes to guarantee that the $L^{2}$-error is accurately evaluated.

\subsection{Two-dimensional results}

We use a sequence of uniformly refined triangular meshes, where each element is split into four triangles.

\subsubsection{Lowest order discretization}

We consider two typical domains for reentrant corners. We start with the Lshaped domain $(-1,1)^{2} \backslash[0,1] \times[-1,0]$ and then consider a slit domain $(-1,1)^{2} \backslash$ $((0,1) \times\{0\})$. In both cases, the prescribed solution is given in polar coordinates by $u(r, \phi)=r^{\alpha} \sin (a \phi)$ where $\alpha, a$ are given parameters. For non-integer $\alpha$, we have $u \in B_{2, \infty}^{1+\alpha}(\Omega)$ by [4, Thm. 2.1]. Moreover, we test the influence of the position $\left(x_{0}, y_{0}\right)$ of the weak singularity at $r=0$ by defining $r^{2}:=\left(x-x_{0}\right)^{2}+\left(y-y_{0}\right)^{2}$. We note that irrespective of the location $\left(x_{0}, y_{0}\right)$ of the singularity on the boundary $\Gamma$, we have $u \in B_{2, \infty}^{1+\alpha}(\Omega) \subset H^{1+\alpha-\varepsilon}(\Omega)$ for any $\varepsilon>0$.

\begin{tabular}{|c|c|c|c|c|c|c|}
\hline & \multicolumn{2}{|c|}{$\begin{array}{c}\left(x_{0}, y_{0}\right)=(0,0) \\
a=\pi / 2\end{array}$} & \multicolumn{2}{|c|}{$\begin{array}{c}\left(x_{0}, y_{0}\right)=(0.5,0) \\
a=\pi\end{array}$} & \multicolumn{2}{|c|}{$\begin{array}{c}\left(x_{0}, y_{0}\right)=(0,1) \\
a=\pi\end{array}$} \\
\hline$\overline{\text { DOFs }}$ & $L^{2}$-error & rate & $L^{2}$-error & rate & $L^{2}$-error & rate \\
\hline 81 & $6.1585 \mathrm{e}-03$ & - & $6.8141 \mathrm{e}-03$ & - & $6.2506 \mathrm{e}-03$ & - \\
\hline 289 & $2.6986 \mathrm{e}-03$ & 1.19 & $2.5648 \mathrm{e}-03$ & 1.41 & $2.1211 \mathrm{e}-03$ & 1.56 \\
\hline 1.089 & $1.1123 \mathrm{e}-03$ & 1.28 & $8.8428 \mathrm{e}-04$ & 1.54 & $6.7413 \mathrm{e}-04$ & 1.65 \\
\hline 4.225 & $4.4037 \mathrm{e}-04$ & 1.34 & $2.9202 \mathrm{e}-04$ & 1.60 & $2.0903 \mathrm{e}-04$ & 1.69 \\
\hline 16.641 & $1.7107 \mathrm{e}-04$ & 1.36 & $9.4164 \mathrm{e}-05$ & 1.63 & $6.4027 \mathrm{e}-05$ & 1.71 \\
\hline 66.049 & $6.5689 \mathrm{e}-05$ & 1.38 & $2.9909 \mathrm{e}-05$ & 1.65 & $1.9471 \mathrm{e}-05$ & 1.72 \\
\hline 263.169 & $2.5030 \mathrm{e}-05$ & 1.39 & $9.4012 \mathrm{e}-06$ & 1.67 & $5.8930 \mathrm{e}-06$ & 1.72 \\
\hline 1.050 .625 & $9.4877 \mathrm{e}-06$ & 1.40 & $2.9328 \mathrm{e}-06$ & 1.68 & $1.7774 \mathrm{e}-06$ & 1.73 \\
\hline 4.198.401 & $3.5834 \mathrm{e}-06$ & 1.40 & $9.0968 \mathrm{e}-07$ & 1.69 & $5.3475 \mathrm{e}-07$ & 1.73 \\
\hline
\end{tabular}

Table 7.1 L-shaped domain, $k=1$ : Influence of the position of singularity for $\alpha=0.75$.

For the L-shaped domain, the shift parameter $s_{0}$ can be taken to be any $s_{0}<$ $2 / 3$. From the theoretical results in Section 3 , we therefore expect the error decay to have a rate of at least $\min (2,1+\alpha-1 / 3)$ uniformly in the position $\left(x_{0}, y_{0}\right)$ of the singularity. Table 7.1 shows the numerical results for $\alpha=0.75$ and $a=2 / 3 \pi$, for which $\min (2,1+\alpha-1 / 3)=1.417$. As it can be seen for $\left(x_{0}, y_{0}\right)=(0,0)$, we observe 
a good agreement with Theorem 3.3 . However for the locations $\left(x_{0}, y_{0}\right)=(0.5,0)$ and $\left(x_{0}, y_{0}\right)=(0,1)$, the rates are substantially better. This can be explained by the more refined analysis of Section 4. Using Corollary 4.3. we expect an improved convergence rate of 1.75 for these cases.

\begin{tabular}{|r||c|c||c|c||c|c|}
\hline \multicolumn{1}{|c||}{} & \multicolumn{2}{c||}{$\alpha=10 / 9$} & \multicolumn{2}{c||}{$\alpha=4 / 3$} & \multicolumn{2}{c|}{$\alpha=3 / 2$} \\
\hline DOFs & $L^{2}$ 2-error & rate & $L^{2}$-error & rate & $L^{2}$-error & rate \\
\hline 81 & $6.5660 \mathrm{e}-03$ & - & $8.6776 \mathrm{e}-03$ & - & $8.9932 \mathrm{e}-03$ & - \\
289 & $2.3309 \mathrm{e}-03$ & 1.49 & $2.8523 \mathrm{e}-03$ & 1.61 & $2.8151 \mathrm{e}-03$ & 1.68 \\
1.089 & $7.3413 \mathrm{e}-04$ & 1.67 & $8.2870 \mathrm{e}-04$ & 1.78 & $7.8034 \mathrm{e}-04$ & 1.85 \\
4.225 & $2.2257 \mathrm{e}-04$ & 1.72 & $2.3073 \mathrm{e}-04$ & 1.84 & $2.0751 \mathrm{e}-04$ & 1.91 \\
16.641 & $6.5650 \mathrm{e}-05$ & 1.76 & $6.2539 \mathrm{e}-05$ & 1.88 & $5.3910 \mathrm{e}-05$ & 1.94 \\
66.049 & $1.9056 \mathrm{e}-05$ & 1.78 & $1.6688 \mathrm{e}-05$ & 1.91 & $1.3835 \mathrm{e}-05$ & 1.96 \\
263.169 & $5.4810 \mathrm{e}-06$ & 1.80 & $4.4099 \mathrm{e}-06$ & 1.92 & $3.5256 \mathrm{e}-06$ & 1.97 \\
1.050 .625 & $1.5690 \mathrm{e}-06$ & 1.80 & $1.1580 \mathrm{e}-06$ & 1.93 & $8.9467 \mathrm{e}-07$ & 1.98 \\
4.198 .401 & $4.4822 \mathrm{e}-07$ & 1.81 & $3.0279 \mathrm{e}-07$ & 1.94 & $2.2641 \mathrm{e}-07$ & 1.98 \\
\hline
\end{tabular}

Table 7.2 L-shaped domain, $k=1$ : Influence of exponent $\alpha$ for $a=2 / 3 \pi$ and $\left(x_{0}, y_{0}\right)=(0,0)$.

Table 7.2 shows the results for $\left(x_{0}, y_{0}\right)=(0,0)$ and $\alpha \in\{10 / 9,4 / 3,3 / 2\}$. From Theorem 3.3. we expect convergence rates of $1.78,2$, and 2 , respectively. The observed numerical rates of $1.81,1.94$, and 1.98 are quite close.

The situation is similar for the slit domain where the regularity of the dual problem is even further reduced. It corresponds to a limiting case of our theory, which, strictly speaking, we did not cover, since the parameter $s_{0}$ of Assumption 1.1 may be taken to be any $s_{0}<1 / 2$. Nevertheless, one expects from Theorem $3.3 \mathrm{a}$ convergence rate close to $\min \{2,1+\alpha-1 / 2\}$. For $\alpha=0.75$ this is 1.25 , which is visible in Table 7.3 for the case $\left(x_{0}, y_{0}\right)=(0,0)$. Again, the better convergence behavior for $\left(x_{0}, y_{0}\right)=(0.5,0)$ and $\left(x_{0}, y_{0}\right)=(0,1)$ can be explained by the theory of Corollary 4.3. which predicts $1+\alpha=1.75$. Table 7.4 shows the results for $\left(x_{0}, y_{0}\right)=(0,0)$ and $\alpha \in\{10 / 9,4 / 3,3 / 2\}$. From Theorem 3.3. we expect convergence rates of 1.61, 1.83 and 2 , respectively. The observed numerical rates of 1.65 , 1.86 , and 1.96 are reasonably close to these predictions.

\begin{tabular}{|r||c|c||c|c||c|c|}
\hline \multicolumn{1}{|c||}{} & \multicolumn{2}{c||}{\begin{tabular}{c}
$\left(x_{0}, y_{0}\right)=(0,0)$ \\
\multicolumn{1}{|c||}{}
\end{tabular}} & \multicolumn{2}{c||}{$\begin{array}{c}\left(x_{0}, y_{0}\right)=(0.5,0) \\
a=\pi / 2\end{array}$} & \multicolumn{2}{c|}{$\begin{array}{c}\left(x_{0}, y_{0}\right)=(0,1) \\
a=\pi\end{array}$} \\
\hline DOFs & $L^{2}$-error & rate & $L^{2}$-error & rate & $L^{2}$-error & rate \\
\hline 97 & $6.1391 \mathrm{e}-03$ & - & $1.1088 \mathrm{e}-02$ & - & $1.0692 \mathrm{e}-02$ & - \\
348 & $2.8187 \mathrm{e}-03$ & 1.12 & $4.1329 \mathrm{e}-03$ & 1.42 & $3.8553 \mathrm{e}-03$ & 1.47 \\
1.315 & $1.2351 \mathrm{e}-03$ & 1.19 & $1.4164 \mathrm{e}-03$ & 1.54 & $1.3388 \mathrm{e}-03$ & 1.53 \\
5.109 & $5.3338 \mathrm{e}-04$ & 1.21 & $4.7830 \mathrm{e}-04$ & 1.57 & $4.4562 \mathrm{e}-04$ & 1.59 \\
20.137 & $2.2846 \mathrm{e}-04$ & 1.22 & $1.4725 \mathrm{e}-04$ & 1.70 & $1.4420 \mathrm{e}-04$ & 1.63 \\
79.953 & $9.7267 \mathrm{e}-05$ & 1.23 & $4.6683 \mathrm{e}-05$ & 1.66 & $4.5843 \mathrm{e}-05$ & 1.65 \\
318.625 & $4.1233 \mathrm{e}-05$ & 1.24 & $1.4761 \mathrm{e}-05$ & 1.66 & $1.4401 \mathrm{e}-05$ & 1.67 \\
1.272 .129 & $1.7428 \mathrm{e}-05$ & 1.24 & $4.3773 \mathrm{e}-06$ & 1.75 & $4.4861 \mathrm{e}-06$ & 1.68 \\
5.083 .777 & $7.3524 \mathrm{e}-06$ & 1.25 & $1.3285 \mathrm{e}-06$ & 1.72 & $1.3889 \mathrm{e}-06$ & 1.69 \\
\hline
\end{tabular}

Table 7.3 Slit domain, $k=1$ : Influence of the position of singularity for $\alpha=0.75$. 


\begin{tabular}{|r||c|c||c|c||c|c|}
\hline \multicolumn{1}{|c||}{} & \multicolumn{2}{c||}{$\alpha=10 / 9$} & \multicolumn{2}{c||}{$\alpha=4 / 3$} & \multicolumn{2}{c|}{$\alpha=3 / 2$} \\
\hline DOFs & $L^{2}$-error & rate & $L^{2}$-error & rate & $L^{2}$-error & rate \\
\hline 97 & $5.7534 \mathrm{e}-03$ & - & $7.3549 \mathrm{e}-03$ & - & $7.5901 \mathrm{e}-03$ & - \\
1.315 & $1.9412 \mathrm{e}-03$ & 1.57 & $2.2414 \mathrm{e}-03$ & 1.71 & $2.1664 \mathrm{e}-03$ & 1.81 \\
5.109 & $6.2583 \mathrm{e}-04$ & 1.63 & $6.4849 \mathrm{e}-04$ & 1.79 & $5.8638 \mathrm{e}-04$ & 1.89 \\
20.137 & $1.9689 \mathrm{e}-04$ & 1.67 & $1.8251 \mathrm{e}-04$ & 1.83 & $1.5450 \mathrm{e}-04$ & 1.92 \\
79.953 & $6.1446 \mathrm{e}-05$ & 1.68 & $5.0718 \mathrm{e}-05$ & 1.85 & $4.0197 \mathrm{e}-05$ & 1.94 \\
318.625 & $6.0229 \mathrm{e}-06$ & 1.68 & $1.4021 \mathrm{e}-05$ & 1.85 & $1.0396 \mathrm{e}-05$ & 1.95 \\
1.272 .129 & $1.9023 \mathrm{e}-06$ & 1.66 & $1.0682 \mathrm{e}-06$ & 1.86 & $6.8978 \mathrm{e}-07$ & 1.96 \\
5.083 .777 & $6.0474 \mathrm{e}-07$ & 1.65 & $2.9514 \mathrm{e}-07$ & 1.86 & $1.7730 \mathrm{e}-07$ & 1.96 \\
\hline
\end{tabular}

Table 7.4 Slit domain, $k=1$ : Influence of exponent $\alpha$ for $a=1 / 2 \pi$ and $\left(x_{0}, y_{0}\right)=(0,0)$

\subsubsection{Second order finite elements}

In this subsection, we test the performance of quadratic finite elements for the Lshaped domain. We use the same type of solution as before and vary the parameter $\alpha$ for $\left(x_{0}, y_{0}\right)=(0,0)$, i.e., the re-entrant corner. Here we expect from our theory a convergence rate of $\min (3, \alpha+1-1 / 3)$. For $\alpha \in\{2.175,2.275,2.375\}$, the observed numerical rates, which are visible in Table 7.5. are very close to the theoretically predicted ones.

\begin{tabular}{|r||c|c||c|c||c|c|}
\hline \multicolumn{1}{|c||}{} & \multicolumn{2}{c|}{$\alpha=2.175$} & \multicolumn{2}{c|}{$\alpha=2.275$} & \multicolumn{2}{c|}{$\alpha=2.375$} \\
\hline DOFs & L2 error & rate & L2 error & rate & L2 error & rate \\
\hline 289 & $2.7565 \mathrm{e}-04$ & - & $2.4570 \mathrm{e}-04$ & - & $2.2177 \mathrm{e}-04$ & - \\
1.089 & $5.1121 \mathrm{e}-05$ & 2.43 & $4.1696 \mathrm{e}-05$ & 2.56 & $3.3912 \mathrm{e}-05$ & 2.71 \\
4.225 & $7.5320 \mathrm{e}-06$ & 2.76 & $5.7319 \mathrm{e}-06$ & 2.86 & $4.3221 \mathrm{e}-06$ & 2.97 \\
16.641 & $1.1051 \mathrm{e}-06$ & 2.77 & $7.8407 \mathrm{e}-07$ & 2.87 & $5.4888 \mathrm{e}-07$ & 2.98 \\
66.049 & $1.5938 \mathrm{e}-07$ & 2.79 & $1.0553 \mathrm{e}-07$ & 2.89 & $6.8762 \mathrm{e}-08$ & 3.00 \\
263.169 & $2.2723 \mathrm{e}-08$ & 2.81 & $1.4044 \mathrm{e}-08$ & 2.91 & $8.5292 \mathrm{e}-09$ & 3.01 \\
1.050 .625 & $3.2138 \mathrm{e}-09$ & 2.82 & $1.8538 \mathrm{e}-09$ & 2.92 & $1.0497 \mathrm{e}-09$ & 3.02 \\
\hline
\end{tabular}

Table 7.5 L-shaped domain, $k=2$ : Influence of $\alpha$ for $a=2 / 3 \pi$ and $\left(x_{0}, y_{0}\right)=(0,0)$.

\subsection{Three-dimensional results}

In the three dimensional setting, we consider a Fichera corner $\Omega:=(-1,1)^{3} \backslash[0,1]^{3}$ and prescribe the smooth solution $u(x, y, z):=\sin ((x+y) \pi) \cos (2 \pi z)$. The inhomogeneous Dirichlet conditions are realized by nodal interpolation. The discretization is based on trilinear finite elements on hexahedra and uniform refinements. Although the dual problem lacks full regularity, Theorem 3.3 asserts that this can be compensated by extra $s_{0}$ regularity of the primal solution to maintain full second order convergence in $L^{2}$.

Table 7.6 shows that we observe numerically already for coarse discretizations the predicted convergence order two, and the theoretical results are confirmed.

We point the reader to Appendix $\mathrm{E}$ for a further numerical results. 


\begin{tabular}{|r|c|c|}
\hline DOF & $L^{2}$-error & rate \\
\hline 316 & 0.075444 & - \\
3.032 & 0.017182 & 1.96 \\
26.416 & 0.0039376 & 2.04 \\
220.256 & 0.00094597 & 2.02 \\
1.798 .336 & 0.00023208 & 2.01 \\
14.532 .992 & $5.7491 \mathrm{e}-05$ & 2.00 \\
\hline
\end{tabular}

Table 7.6 Fichera corner, $k=1: L^{2}$-error for a smooth solution. 
A Coverings

In this appendix, the distance $\operatorname{dist}(x, M)$ for some set $M$ appears frequently. For notational convenience, we set $\operatorname{dist}(x, \emptyset)=1$ to include the degenerate case $M=\emptyset$.

We quote from [18, Lemma A.1]:

Lemma A.1 Let $\Omega \subset \mathbb{R}^{d}$ be bounded open and $M=\bar{M}$ be a closed set. Fix $c \in(0,1)$ and $\varepsilon \in(0,1)$ such that

$$
1-c(1+\varepsilon)=: c_{0}>0 \text {. }
$$

For each $x \in \Omega$, let $B_{x}:=\bar{B}_{c \operatorname{dist}(x, M)}(x)$ be the closed ball of radius $c \operatorname{dist}(x, M)$ centered at $x$, and let $\widehat{B}_{x}:=\bar{B}_{(1+\varepsilon) c \operatorname{dist}(x, M)}(x)$ denote the stretched (closed) ball of radius $(1+\varepsilon) c \operatorname{dist}(x, M)$ also centered at $x$.

Then there exists a countable set $x_{i} \in \Omega, i \in \mathbb{N}$, and a constant $N \in \mathbb{N}$ depending solely on the spatial dimension $d$ with the following properties:

1. (covering property) $\cup_{i \in \mathbb{N}} B_{x_{i}} \supset \Omega$;

2. (finite overlap on $\Omega$ ) for each $x \in \Omega$, there holds $\operatorname{card}\left\{i \mid x \in \widehat{B}_{x_{i}}\right\} \leq N$.

Proof [18, Lemma A.1] assumed that $M \subset \bar{\Omega}$. However, an inspection of the proof shows that this is not necessary.

Before we proceed with variants of the covering result of Lemma A.1 we introduce the notation of sectorial neighborhoods relative a singular set $M$ :

Definition A.2 (sectorial neighborhood) Let $e, M \subset \mathbb{R}^{d}$ and $\widetilde{c}>0$. Then

$$
S_{e, M, \widetilde{c}}:=\cup_{x \in e} B_{\widetilde{c} \operatorname{dist}(x, M)}(x)
$$

is a sectorial neighborhood of the set e relative to the singular set $M$.

We are interested in coverings of lower-dimensional manifolds by balls whose centers are located on these manifolds:

Lemma A.3 Let $d \in \mathbb{N}$ and $1 \leq d^{\prime}<d$. Let $\omega \subset \mathbb{R}^{d^{\prime}}$ and let $\Omega \subset \mathbb{R}^{d}$ be the canonical embedding of $\omega$ into $\mathbb{R}^{d}$, i.e., $\Omega:=\omega \times\{0\} \times \cdots \times\{0\} \subset \mathbb{R}^{d}$. Assume the hypotheses and notation of Lemma A.1. Then there are $\widetilde{c}>0, N>0$, and a collection of balls $B_{x_{i}}, i \in \mathbb{N}$, as described in Lemma $A .1$ such that

(i) (covering property for $\Omega) \cup_{i \in \mathbb{N}} B_{x_{i}} \supset \Omega$.

(ii) (covering property for a sectorial neighborhood of $\Omega$ ) $\cup_{i \in \mathbb{N}} B_{x_{i}} \supset S_{\Omega, M, \widetilde{c}}$.

(iii) (finite overlap property on $\mathbb{R}^{d}$ ) for each $x \in \mathbb{R}^{d}$, there holds card $\{i \mid x \in$ $\left.\widehat{B}_{x_{i}}\right\} \leq N$.

Proof We employ the result of Lemma A.1 for the lower-dimensional manifold $\omega$ noting that $B_{x} \cap \omega$ is a ball in $\mathbb{R}^{d^{\prime}}$. In order to be able to ensure the covering condition for the sectorial neighborhood of $\Omega$ stated in (iii), we introduce the auxiliary balls $B_{x}^{\prime}:=\bar{B}_{c / 2 \operatorname{dist}(x, M)}(x)$ of half the radius. Applying Lemma A.1 with these balls $B_{x}^{\prime}$ and the stretched balls $\widehat{B}_{x}$ therefore produces a collection of centers $x_{i} \in \Omega, i \in \mathbb{N}$, such that

1. $B_{x_{i}}^{\prime} \cap \Omega$ covers $\Omega$; 
2. for the stretched balls $\widehat{B}_{x_{i}}$, we have a finite overlap property on $\Omega$ :

$$
\forall x \in \Omega: \quad \operatorname{card}\left\{i \mid x \in \widehat{B}_{x_{i}}\right\} \leq N .
$$

We next see that the balls $\widehat{B}_{x_{i}}$ even have the following, stronger finite overlap property:

$$
\forall x \in \mathbb{R}^{d}: \quad \operatorname{card}\left\{i \mid x \in \widehat{B}_{x_{i}}\right\} \leq N .
$$

To see this, define the infinite cylinders $\widehat{C}_{x_{i}}:=\left\{x \mid \pi_{d^{\prime}}(x) \in \widehat{B}_{x_{i}} \cap \Omega\right\}$, where $\pi_{d^{\prime}}$ is the canonical projection onto the hyperplane $\left\{x=\left(x_{1}, \ldots, x_{d}\right) \in \mathbb{R}^{d} \mid x_{d^{\prime}+1}=\right.$ $\left.\cdots=x_{d}=0\right\}$. Clearly, $\widehat{B}_{x_{i}} \subset \widehat{C}_{x_{i}}$. These infinite cylinders have a finite overlap property by A.2 as can be seen by writing any $x \in \mathbb{R}^{d}$ in the form $x=\left(\pi_{d^{\prime}}(x), x^{\prime}\right)$ for some $x^{\prime} \in \mathbb{R}^{d-d^{\prime}}$ and then noting that $x \in \widehat{C}_{x_{i}}$ implies $\pi_{d^{\prime}}(x) \in \widehat{B}_{x_{i}} \cap \Omega$.

Is remains to see that the balls $B_{x_{i}}$ cover a sectorial neighborhood of $\Omega$. To that end, we note that the balls $B_{x_{i}}^{\prime}$ cover $\Omega$. Furthermore, for each $x \in \Omega$, we pick $x_{i}$ such that $x \in B_{x_{i}}^{\prime} \subset B_{x_{i}}$. Since the radius of $B_{x_{i}}$ is twice that of $B_{x_{i}}^{\prime}$, we even have $B_{c / 2 \operatorname{dist}\left(x_{i}, M\right)}(x) \subset B_{x_{i}}$. Furthermore, by $c \in(0,1)$, we have $0<(1-c / 2) \operatorname{dist}\left(x_{i}, M\right) \leq \operatorname{dist}(x, M) \leq(1+c / 2) \operatorname{dist}\left(x_{i}, M\right)$. Therefore, there is $\widetilde{c}>0$ such that $B_{\widetilde{c} \operatorname{dist}(x, M)}(x) \subset B_{x_{i}}$ and thus

$$
\cup_{x \in \Omega} B_{\widetilde{c} \operatorname{dist}(x, M)}(x) \subset \cup_{i} B_{x_{i}} .
$$

We next show covering theorems for polygons and polyhedra. In the interest of clarity of presentation, we formulate two separate results. Before doing so, we point out that balls with center located on the boundary of the polygon/polyhedron $\Omega$ will feature importantly so that the intersection of this ball with $\Omega$ will be of interest. We therefore introduce the following notions:

\section{Definition A.4 (solid angles and dihedral angles)}

1. Let $\Omega \subset \mathbb{R}^{2}$ be a Lipschitz polygon. Let $A$ be a vertex where the edges $e_{1}, e_{2}$ meet. We say that the set $B_{\varepsilon}(A) \cap \Omega$ is a solid angle, if $\partial\left(B_{\varepsilon}(A) \cap \Omega\right) \cap \partial \Omega$ is contained in $\{A\} \cup e_{1} \cup e_{2}$.

2. Let $\Omega \subset \mathbb{R}^{3}$ be a Lipschitz polyhedron. Let $A$ be a vertex of $\Omega$. We say that the set $B_{\varepsilon}(A) \cap \Omega$ is a solid angle, if $\partial\left(B_{\varepsilon}(A) \cap \Omega\right) \cap \partial \Omega$ is contained in the union of $\{A\}$ and the edges and faces meeting at $A$.

3. Let $\Omega \subset \mathbb{R}^{3}$ be a Lipschitz polyhedron. Let $e$ be an edge of $\Omega$, which is shared by the faces $f_{1}, f_{2}$. Let $x \in e$. We say that the set $B_{\varepsilon}(x) \cap \Omega$ is a dihedral angle, if $\partial\left(B_{\varepsilon}(x) \cap \Omega\right) \cap \partial \Omega$ is contained in $e \cup f_{1} \cup f_{2}$.

Theorem A.5 Let $\Omega \subset \mathbb{R}^{2}$ be a bounded Lipschitz polygon with vertices $A_{j}, j=$ $1, \ldots, J$, and edges $\mathcal{E}$. Let $M \subset\left\{A_{1}, \ldots, A_{J}\right\}$. Set $\mathcal{A}^{\prime}:=\left\{A_{1}, \ldots, A_{J}\right\} \backslash M$ and fix $\varepsilon \in(0,1)$.

(i) There is a sectorial neighborhood $S_{\mathcal{A}^{\prime}, M, \widetilde{c}}$ of the vertices $\mathcal{A}^{\prime}$ and a constant $c \in(0,1)$ such that $S_{\mathcal{A}^{\prime}, M, \widetilde{c}}$ is covered by balls $B_{i}:=\bar{B}_{c \operatorname{dist}\left(x_{i}, M\right)}\left(x_{i}\right)$ with centers $x_{i} \in \mathcal{A}^{\prime}$. Furthermore, the stretched balls $\widehat{B}_{i}:=\bar{B}_{(1+\varepsilon) c \operatorname{dist}\left(x_{i}, M\right)}\left(x_{i}\right)$ are solid angles and satisfy a finite overlap property on $\mathbb{R}^{2}$. 
(ii) Fix a sectorial neighborhood $\mathcal{U}:=S_{\mathcal{A}^{\prime}, M, c^{\prime}}$ of the vertices $\mathcal{A}^{\prime}$. For each edge $e \in \mathcal{E}$, there is a sectorial neighborhood $S_{e, M, \widetilde{c}}$ and a constant $c \in(0,1)$ such that $S_{e, M, \widetilde{c}} \backslash \mathcal{U}$ is covered by balls $B_{i}=\bar{B}_{c \operatorname{dist}\left(x_{i}, M\right)}\left(x_{i}\right)$ whose centers $x_{i}$ are located on e. Furthermore, the stretched balls $\widehat{B}_{i}=\bar{B}_{(1+\varepsilon) c \operatorname{dist}\left(x_{i}, M\right)}\left(x_{i}\right)$ satisfy a finite overlap property on $\mathbb{R}^{2}$ and are such that each $\widehat{B}_{i} \cap \Omega$ is a half-disk.

(iii) Fix a sectorial neighbood $\mathcal{U}:=S_{\mathcal{E}, M, c^{\prime}}$ of the edges $\mathcal{E}$. There is $c \in(0,1)$ such that $\Omega \backslash \mathcal{U}$ is covered by balls $B_{i}=\bar{B}_{c \operatorname{dist}\left(x_{i}, M\right)}\left(x_{i}\right)$ such that the stretched balls $\widehat{B}_{i}=\bar{B}_{(1+\varepsilon) c \operatorname{dist}\left(x_{i}, M\right)}\left(x_{i}\right)$ are completely contained in $\Omega$ and satisfy a finite overlap property on $\mathbb{R}^{2}$.

Proof The assertion (ii) is almost trivial and only included to emphasize the structure of the arguments. Assertions (ii), (iii) follow from suitable applications of Lemmas A.3 and A.1

The 3D variant of Theorem A.5 is formulated in Theorem A.6 We emphasize that the "singular" set $M$ need not be the union of all edges and vertices but can be just a subset. We also emphasize that it is not necessarily related to the notion of "singular set" in Definition 2.5 although it is used in this way. The key property of the covering balls is again such that the centers are either a) in $\Omega$ (in which case the stretched ball is contained in $\Omega$ ); or b) on a face (in which case the stretched ball $\widehat{B}_{i}$ is such that $\widehat{B}_{i} \cap \Omega$ is a half-ball); or c) on an edge in which case $\widehat{B}_{i} \cap \Omega$ is a dihedral angle (see Definition A.4); or d) in a vertex in which case $\widehat{B}_{i} \cap \Omega$ is a solid angle (see Definition A.4).

Theorem A.6 Let $\Omega \subset \mathbb{R}^{3}$ be a Lipschitz polyhedron with faces $\mathcal{F}$, edges $\mathcal{E}$, and vertices $\mathcal{A}$. Let $M_{\mathcal{A}} \subset \mathcal{A}$ and $M_{\mathcal{E}} \subset \mathcal{E}$. Let $M=\bar{M}=\overline{M_{\mathcal{A}} \cup M_{\mathcal{E}}}$ and fix $\varepsilon \in(0,1)$. Let $\mathcal{A}^{\prime}:=\{A \in \mathcal{A} \mid A \notin M\}$ be the vertices not in $M$ and $\mathcal{E}^{\prime}:=\{e \in \mathcal{E} \mid \bar{e} \cap M=\emptyset\}$ be the edges not abutting $M$. Then:

(i) (non-singular vertices) There is a sectorial neighborhood $S_{\mathcal{A}^{\prime}, M, \widetilde{c}}$ of the vertices in $\mathcal{A}^{\prime}$ and a constant $c \in(0,1)$ such that $S_{\mathcal{A}^{\prime}, M, \widetilde{c}}$ is covered by balls $B_{i}:=\bar{B}_{c \operatorname{dist}\left(x_{i}, M\right)}\left(x_{i}\right)$ with centers $x_{i} \in \mathcal{A}^{\prime}$. Furthermore, the stretched balls $\widehat{B}_{i}:=\bar{B}_{(1+\varepsilon) c \operatorname{dist}\left(x_{i}, M\right)}\left(x_{i}\right)$ are solid angles and satisfy a finite overlap property on $\mathbb{R}^{3}$.

(ii) (non-singular edges) Fix a sectorial neighborhood $\mathcal{U}:=S_{\mathcal{A}^{\prime}, M, c^{\prime}}$ of $\mathcal{A}^{\prime}$. For each edge $e \in \mathcal{E}^{\prime}$, there is a sectorial neighborhood $S_{e, M, \widetilde{c}}$ and a constant $c \in(0,1)$ such that $S_{e, M, \widetilde{c}} \backslash \mathcal{U}$ is covered by balls $B_{i}=\bar{B}_{c \operatorname{dist}\left(x_{i}, M\right)}\left(x_{i}\right)$ whose centers $x_{i}$ are located on e. Furthermore, the stretched balls $\widehat{B}_{i}=$ $\bar{B}_{(1+\varepsilon) c \operatorname{dist}\left(x_{i}, M\right)}\left(x_{i}\right)$ satisfy a finite overlap property on $\mathbb{R}^{3}$ and $\widehat{B}_{i} \cap \Omega$ is a dihedral angle.

(iii) (faces) Fix a sectorial neighbood $\mathcal{U}:=S_{\mathcal{E}, M, c^{\prime}}$ of $\mathcal{E}$. There is a sectorial neighborhood $S_{\mathcal{F}, M, \widetilde{c}}$ and a constant $c \in(0,1)$ such that $S_{\mathcal{F}, M, \widetilde{c}} \backslash \mathcal{U}$ is covered by balls $B_{i}=\bar{B}_{c \operatorname{dist}\left(x_{i}, M\right)}\left(x_{i}\right)$ with centers $x_{i} \in \partial \Omega$. Furthermore, the stretched balls $\widehat{B}_{i}=\bar{B}_{(1+\varepsilon) c \operatorname{dist}\left(x_{i}, M\right)}\left(x_{i}\right)$ satisfy a finite overlap property on $\mathbb{R}^{3}$ and $\widehat{B}_{i} \cap \Omega$ is a half-ball.

(iv) (interior) Fix a sectorial neighbood $\mathcal{U}:=S_{\mathcal{F}, M, c^{\prime}}$ of $\mathcal{F}$, where $\mathcal{F}$ is the set of faces. Then there is $c \in(0,1)$ such that $\Omega \backslash \mathcal{U}$ is covered by balls $B_{i}=$ 
$\bar{B}_{c \operatorname{dist}\left(x_{i}, M\right)}\left(x_{i}\right)$ with centers $x_{i} \in \Omega$. Furthermore, the stretched balls $\widehat{B}_{i}=$ $\bar{B}_{(1+\varepsilon) c \operatorname{dist}\left(x_{i}, M\right)}\left(x_{i}\right)$ satisfy a finite overlap property on $\mathbb{R}^{3}$ and $\widehat{B}_{i} \subset \Omega$.

Proof Follows from Lemmas A.3 and A.1 


\section{B Details for the extension of the results of [18].}

The arguments used in the proof of Theorem 5.1 rely on techniques developed in [18. A feature of the analysis here that was not present in 18 is the bidual problem with right-hand side $\widetilde{\delta}_{\Gamma}^{-1}\left(w-w_{h}\right)$ that allowed us to estimate $w-w_{h}$ in a weighted space. This technical issue was circumvented in [18] by assuming convexity of $\Omega$ so that optimal order $L^{2}$-estimates could be cited from the literature. The corresponding bidual problem can be analyzed in the mortar setting of [18] as well. The end result is then Theorem B.1 which states that the convexity assumption in [18] can be relaxed to the validity of Assumption 1.1] for the Poisson problem, i.e., [18, (5.2)].

In the interest of brevity, we employ in this appendix the notation of [18] and assume the reader's familiarity with [18.

It will be useful to write $H_{p w}^{s}(\Gamma)$ for the space given by the broken Sobolev norm on the skeleton $\Gamma$, i.e., the Sobolev norm is understood facewise. Furthermore, we will write $\|\cdot\|_{H^{1}}$ for the broken $H^{1}$-norm, i.e., $\|\cdot\|_{H^{1}}^{2}=\sum_{i}\|\cdot\|_{H^{1}\left(\Omega_{i}\right)}^{2}$. We also introduce the $L^{2}$-projection $\Pi_{M_{h}}^{L^{2}}: L^{2}(\Gamma) \rightarrow M_{h}$ and recall that, since $M_{h}$ is a product space based on the faces, it inherits from [18, (A2)] the approximation property

$$
\left\|z-\Pi_{M_{h}}^{L^{2}} z\right\|_{L^{2}(\Gamma)} \leq C h^{s}\|z\|_{H_{p w}^{s}(\Gamma)}, \quad s \in[0, k] .
$$

The main result is:

Theorem B.1 In [18, Thms. 2.1, 2.5], the assumption of convexity of $\Omega$ can be replaced with [18, Assumption (5.2)].

Proof We will only sketch the modifications entailed by the weakenend regularity assumptions.

Proof of [18, Thm. 2.1]: The starting point is the error representation [18, (6.2)], which consists of three terms:

$$
a\left(w-w_{h}, u-P_{h} u\right)+b\left(w-w_{h}, \lambda-\mu_{h}\right)+b\left(u-P_{h} u, \lambda_{w}-\tilde{\mu}_{h}\right),
$$

where $\mu_{h}, \tilde{\mu}_{h} \in M_{h}$ are arbitrary. The first term in (B.2) can be estimated as in 18 , Proof of Thm. 2.1] in view of the generalization of [18, Lemma 5.5] given below as Lemma B.3. The third term in (B.2) can again be estimated as in [18, (6.4)] since Lemma B.2 below provides the estimate $\inf _{\tilde{\mu}_{h} \in M_{h}}\left\|\lambda_{w}-\tilde{\mu}_{h}\right\|_{L^{2}(\Gamma)} \lesssim h^{1 / 2}\|v\|_{L^{2}(\Omega)}$. The second term in (B.2) requires a modification of the procedure in [18, (6.3)]. Taking $\mu_{h}=\Pi_{M_{h}}^{L^{2}} \lambda$ in [18, (6.3)] yields

$$
\begin{aligned}
b\left(w-w_{h}, \lambda-\Pi_{M_{h}}^{L^{2}} \lambda\right) & =\int_{\Gamma}\left[w-w_{h}\right]\left(\lambda-\Pi_{M_{h}}^{L^{2}} \lambda\right) \\
& =\int_{\Gamma}\left(\left[w-w_{h}\right]-\Pi_{M_{h}}^{L^{2}}\left[w-w_{h}\right]\right)\left(\lambda-\Pi_{M_{h}}^{L^{2}} \lambda\right) \\
& \lesssim h^{1 / 2}\left\|\left[w-w_{h}\right]\right\|_{H_{p w}^{1 / 2}(\Gamma)}\left\|\lambda-\Pi_{M_{h}}^{L^{2}}\right\|_{L^{2}(\Gamma)} \\
& \lesssim h^{1 / 2}\left\|w-w_{h}\right\|_{H^{1}}\left\|\lambda-\Pi_{M_{h}}^{L^{2}} \lambda\right\|_{L^{2}(\Gamma)} \\
& \lesssim h^{1 / 2+k}\left\|w-w_{h}\right\|_{H^{1}}\|\lambda\|_{H_{p w}^{k}(\Gamma)}
\end{aligned}
$$


the last step followed from (B.1). The proof is completed with the aid of Lemma B.2 and the trace estimate $\|\lambda\|_{H_{p w}^{k}(\Gamma)} \lesssim\|u\|_{B_{2,1}^{k+3 / 2}(\Omega)}$.

Proof of [18, Thm. 2.5]: The proof stands as given in [18].

Lemma B.2 Assume that $\Omega$ satisfies [18, Assumption (5.2)]. Then, for $v \in$ $L^{2}\left(S_{h}\right) \subset L^{2}(\Omega)$ and $w:=T^{D}(v)$ and the corresponding Lagrange multiplier $\lambda_{w}$ defined facewise by $\left.\lambda_{w}\right|_{\gamma_{l}}=-\left.\partial_{n} w\right|_{\Omega_{s(l)}}$ and the corresponding mortar approximation $w_{h}$ of $w$ there holds:

$$
\begin{aligned}
& \sqrt{\sum_{i}\left\|w-w_{h}\right\|_{H^{1}\left(\Omega_{i}\right)}^{2}}=\left\|w-w_{h}\right\|_{H^{1}} \lesssim h\|v\|_{L^{2}(\Omega)}, \\
&\left\|\lambda_{w}-\Pi_{M_{h}}^{L^{2}} \lambda_{w}\right\|_{L^{2}(\Gamma)} \lesssim h^{1 / 2}\|v\|_{L^{2}(\Omega)} .
\end{aligned}
$$

Proof We start with the proof of (B.3). It results from standard convergence theory for mortar methods as follows. [18, Assumption (5.2)] provides $w \in H^{3 / 2+\varepsilon}(\Omega)$ for some $\varepsilon>0$ together with $\|w\|_{H^{3 / 2+\varepsilon}(\Omega)} \leq C\|v\|_{H^{-1 / 2+\varepsilon}(\Omega)}$. The Lagrange multiplier $\lambda_{w}$ is given facewise by the expression $\left.\lambda_{w}\right|_{\gamma_{l}}=-\left.\partial_{n} w\right|_{\Omega_{s(l)}} \in H^{\varepsilon}\left(\gamma_{l}\right)$ together with the estimate $\left\|\lambda_{w}\right\|_{H_{p w}^{\varepsilon}(\Gamma)} \lesssim\|v\|_{H^{-1 / 2+\varepsilon}(\Omega)}$. The standard convergence theory for mortar methods (as worked out, e.g., in [8, Prop. 2.3]) then gives

$$
\left\|w-w_{h}\right\|_{H^{1}} \lesssim h^{1 / 2+\varepsilon}\|w\|_{H^{3 / 2+\varepsilon}(\Omega)} \lesssim h^{1 / 2+\varepsilon}\|v\|_{H^{-1 / 2+\varepsilon}(\Omega)} .
$$

The proof of $(\mathrm{B} .3)$ is complete if we can show that

$$
\|v\|_{H^{-1 / 2+\varepsilon}(\Omega)} \lesssim h^{1 / 2-\varepsilon}\|v\|_{L^{2}(\Omega)} .
$$

This last estimate exploits $\operatorname{supp} v \subset S_{h}$ and follows by interpolation arguments similar to those employed in the proof of [18, Lemma 5.2]: Define $\theta=1-2 \varepsilon$ (we assume $\varepsilon<1 / 2)$. Then $H^{-1 / 2+\varepsilon}(\Omega)=\left(H^{1 / 2-\varepsilon}(\Omega)\right)^{\prime}=\left(\left(L^{2}(\Omega), B_{2,1}^{1 / 2}(\Omega)\right)_{\theta, 2}\right)^{\prime}=$ $\left(\left(B_{2,1}^{1 / 2}(\Omega)\right)^{\prime}, L^{2}(\Omega)\right)_{\theta, 2}$, so that the interpolation inequality yields $\|v\|_{H^{-1 / 2+\varepsilon}(\Omega)} \lesssim$ $\|v\|_{\left(B_{2,1}^{1 / 2}(\Omega)\right)^{\prime}}^{\theta}\|v\|_{L^{2}(\Omega)}^{1-\theta}$. The argument is completed by noting in view of supp $v \subset$ $S_{h}$ that

$$
\|v\|_{L^{2}(\Omega)} \leq\|v\|_{L^{2}(\Omega)} \quad \text { and } \quad\|v\|_{\left(B_{2,1}^{1 / 2}(\Omega)\right)^{\prime}} \leq C \sqrt{h}\|v\|_{L^{2}(\Omega)},
$$

so that $\|v\|_{H^{-1 / 2+\varepsilon}(\Omega)} \lesssim h^{(1-\theta) / 2}\|v\|_{L^{2}(\Omega)} \lesssim h^{1 / 2-\varepsilon}\|v\|_{L^{2}(\Omega)}$. This proves (B.5).

The bound (B.4) follows from (B.1) and $\left\|\lambda_{w}-\Pi_{M_{h}}^{L^{2}} \lambda_{w}\right\|_{L^{2}(\Gamma)} \leq C h^{\varepsilon}\left\|\lambda_{w}\right\|_{H_{p w}^{\varepsilon}(\Gamma)} \lesssim$ $h^{\varepsilon}\|w\|_{H^{3 / 2+\varepsilon}(\Omega)} \lesssim h^{\varepsilon}\|v\|_{H^{-1 / 2+\varepsilon}(\Omega)}$. An appeal to (B.5) finishes the proof.

We generalize [18, Lemma 5.5]:

Lemma B.3 [generalizations of [18, Lemma 5.5]] Assume that $\Omega$ satisfies [18, Assumption (5.2)]. Then, for $v \in L^{2}\left(S_{h}\right) \subset L^{2}(\Omega)$ and $w:=T^{D}(v)$ and the mortar approximation $w_{h}$ of $w$, there holds

$$
\left\|\nabla\left(w-w_{h}\right)\right\|_{L^{2}\left(\Gamma ; L^{1}\right)} \leq C h^{3 / 2}\left(1+\delta_{k, 1}|\ln h|\right)\|v\|_{L^{2}(\Omega)} .
$$


Proof Inspection of the proof of [18, Lemma 5.5] shows that we have to estimate the following two terms:

$$
T_{1}:=\int_{\tau=0}^{\tilde{c} h}\left\|\nabla\left(w-w_{h}\right)\right\|_{L^{2}\left(\gamma_{\tau}\right)} \quad \text { and } \quad T_{2}:=\int_{\tau=\tilde{c} h}^{D}\left\|\nabla\left(w-w_{h}\right)\right\|_{L^{2}\left(\gamma_{\tau}\right)} .
$$

Estimating $\mathbf{T}_{\mathbf{1}}$ : Inspection of the proof of [18, Lemma 5.5] shows that

$$
T_{1} \leq \sqrt{h}\left\|\nabla\left(w-w_{h}\right)\right\|_{L^{2}(S(0, \tilde{c} h))} \leq \sqrt{h}\left\|\nabla\left(w-w_{h}\right)\right\|_{L^{2}\left(\Omega_{i}\right)} .
$$

We conclude together with (B.3)

$$
T_{1} \leq C h^{3 / 2}\|v\|_{L^{2}(\Omega)} .
$$

We now turn to estimating $T_{2}$. As in the proof of [18, Lemma 5.5], we consider the lowest order case $k=1$ and the higher order cases $k>1$ separately.

Estimating $\mathbf{T}_{\mathbf{2}}$ for $\mathbf{k}=1$ : Inspection of the proof of [18, Lemma 5.5] gives (cf. [18, eqn. (5.13)])

$$
\begin{aligned}
& \int_{\tau=\tilde{c} h}^{D}\left\|\nabla\left(w-w_{h}\right)\right\|_{L^{2}\left(\gamma_{\tau}\right)} d \tau \\
& \quad \lesssim|\ln h|^{1 / 2}\left(\left\|\delta_{\Gamma}^{1 / 2} \nabla\left(w-I_{h}^{1} w\right)\right\|_{L^{2}\left(\mathcal{C}^{\prime} \backslash S_{c_{1} h}\right)}+\left\|\delta_{\Gamma}^{-1 / 2}\left(w-w_{h}\right)\right\|_{L^{2}\left(\mathcal{C}^{\prime} \backslash S_{c_{1} h}\right)}\right) .
\end{aligned}
$$

The term $w-I_{h}^{1} w$ is estimated as in the proof of [18 Lemma 5.5] with the aid of (weighted) $H^{2}$-regularity asserted in [18, Lemma 5.4] and the estimate $\|w\|_{B_{2, \infty}^{3 / 2}(\Omega)} \lesssim \sqrt{h}\|v\|_{L^{2}(\Omega)}$ of [18, Lemma 5.2]. In total, we get

$$
\left\|\delta_{\Gamma}^{1 / 2} \nabla\left(w-I_{h}^{1} w\right)\right\|_{L^{2}\left(\mathcal{C}^{\prime} \backslash S_{c_{1} h}\right)} \lesssim|\ln h|^{1 / 2} h^{3 / 2}\|v\|_{L^{2}(\Omega)} .
$$

The second contribution of the right-hand side of (B.6) has to be treated with more care than in the proof of [18, Lemma 5.5], where the convexity of $\Omega$ was conveniently exploited in order to control $\left\|w-w_{h}\right\|_{L^{2}(\Omega)}$; more precisely, we do not have full $H^{2}$-regularity but only the limited shift theorem of [18, Assumption (5.2)]. In order to control the term $\left\|\delta_{\Gamma}^{-1 / 2}\left(w-w_{h}\right)\right\|_{L^{2}\left(\mathcal{C}^{\prime} \backslash S_{c_{1} h}\right)}$ appearing on the right-hand side of (B.6), we proceed by yet another duality argument. Let $\psi:=T^{D}\left(\widetilde{\delta}_{\Gamma}^{-1}\left(w-w_{h}\right)\right)$, where $\widetilde{\delta}_{\Gamma}$ is the regularized distance function $\widetilde{\delta}_{\Gamma} \sim h+\delta_{\Gamma}$. Note that $\widetilde{\delta}_{\Gamma} \sim \delta_{\Gamma}$ on $\mathcal{C}^{\prime} \backslash S_{c_{1} h}$. Denote by $\lambda_{\psi}$ the Lagrange multiplier for $\psi$, i.e., $\left.\lambda_{\psi}\right|_{\gamma_{l}}=-\left.\partial_{n} \psi\right|_{\Omega_{s(l)}}$. From Lemma B.4 we have for some $\varepsilon>0$ given by the stipulated shift theorem ([18, Assumption (5.2)])

$$
\begin{aligned}
\|\psi\|_{B_{2, \infty}^{3 / 2}(\Omega)} & \lesssim|\ln h|^{1 / 2}\left\|\widetilde{\delta}_{\Gamma}^{-1 / 2}\left(w-w_{h}\right)\right\|_{L^{2}(\Omega)}, \\
\left\|\lambda_{\psi}\right\|_{H_{p w}^{\varepsilon}(\Gamma)}+\|\psi\|_{H^{3 / 2+\varepsilon}(\Omega)} & \left.\lesssim h\right|^{-\varepsilon}\left\|\widetilde{\delta}_{\Gamma}^{-1 / 2}\left(w-w_{h}\right)\right\|_{L^{2}(\Omega)} .
\end{aligned}
$$

The pair $\left(\psi, \lambda_{\psi}\right)$ solves the following saddle point problem:

$$
\begin{aligned}
a(z, \psi)+b\left(z, \lambda_{\psi}\right) & =\left(z, \tilde{\delta}^{-1}\left(w-w_{h}\right)\right) \quad \forall z \in\left\{z \in \prod_{i} H^{1}\left(\Omega_{i}\right)|z|_{\partial \Omega}=0\right\} \\
b(\psi, q) & =0 \quad \forall q \in \prod_{i} H_{00}^{1 / 2}\left(\gamma_{i}\right) .
\end{aligned}
$$


The Galerkin orthogonality satisfied by $w-w_{h}$ reads for arbitrary $I \psi \in V_{h}$

$$
a\left(w-w_{h}, I \psi\right)+b\left(I \psi, \lambda_{w}\right)=0 .
$$

Hence, we get by taking $z=w-w_{h}$ in (B.9a)

$$
\begin{aligned}
\left\|\widetilde{\delta}_{\Gamma}^{-1 / 2}\left(w-w_{h}\right)\right\|_{L^{2}(\Omega)}^{2} & =a\left(w-w_{h}, \psi\right)+b\left(w-w_{h}, \lambda_{\psi}\right) \\
& =a\left(w-w_{h}, \psi-I \psi\right)-b\left(I \psi, \lambda_{w}\right)+b\left(w-w_{h}, \lambda_{\psi}\right) .
\end{aligned}
$$

We estimate $\left|a\left(w-w_{h}, \psi-I \psi\right)\right| \lesssim\left\|w-w_{h}\right\|_{H^{1}}\|\psi-I \psi\|_{H^{1}}$. Since $I \psi \in V_{h}$ and $[\psi]=0$ :

$$
\begin{aligned}
\left|b\left(I \psi, \lambda_{w}\right)\right| & =b\left(\psi-I \psi, \lambda_{w}-\Pi_{M_{h}}^{L^{2}} \lambda_{w}\right) \mid \lesssim\|\psi-I \psi\|_{L^{2}(\Gamma)}\left\|\lambda_{w}-\Pi_{M_{h}}^{L^{2}} \lambda_{w}\right\|_{L^{2}(\Gamma)} \\
& \leq\|\psi-I \psi\|_{L^{2}(\Omega)}^{1 / 2}\|\psi-I \psi\|_{H^{1}}^{1 / 2} h^{\varepsilon}\left\|\lambda_{w}\right\|_{H_{p w}^{\varepsilon}(\Gamma)},
\end{aligned}
$$

where, in the last step, we employed the multiplicative trace inequality and the approximation property (B.1). For the term $b\left(w-w_{h}, \lambda_{\psi}\right)$, we employ again that $[w]=0$ and that $w_{h} \in V_{h}$ to get

$$
\begin{aligned}
b\left(w-w_{h}, \lambda_{\psi}\right) & =\int_{\Gamma}\left[w-w_{h}\right]\left(\lambda_{\psi}-\Pi_{M_{h}}^{L^{2}} \lambda_{\psi}\right) \\
& =\int_{\Gamma}\left(\left[w-w_{h}\right]-\Pi_{M_{h}}^{L^{2}}\left[w-w_{h}\right]\right)\left(\lambda_{\psi}-\Pi_{M_{h}}^{L^{2}} \lambda_{\psi}\right) \\
& \lesssim h^{1 / 2}\left\|\left[w-w_{h}\right]\right\|_{H_{p w}^{1 / 2}(\Gamma)} h^{\varepsilon}\left\|\lambda_{\psi}\right\|_{H_{p w}^{\varepsilon}(\Gamma)} .
\end{aligned}
$$

Noting $\left\|\left[w-w_{h}\right]\right\|_{H_{p w}^{1 / 2}(\Gamma)} \lesssim\left\|w-w_{h}\right\|_{H^{1}}$ we obtain by inserting the above estimates in (B.10)

$$
\begin{aligned}
& \left\|\widetilde{\delta}^{-1 / 2}\left(w-w_{h}\right)\right\|_{L^{2}(\Omega)}^{2} \lesssim \\
& \quad\left\|w-w_{h}\right\|_{H^{1}}\|\psi-I \psi\|_{H^{1}}+\|\psi-I \psi\|_{L^{2}}^{1 / 2}\|\psi-I \psi\|_{H^{1}}^{1 / 2} h^{\varepsilon}\left\|\lambda_{w}\right\|_{H_{p w}^{\varepsilon}(\Gamma)} \\
& \quad+h^{1 / 2}\left\|w-w_{h}\right\|_{H^{1}} h^{\varepsilon}\left\|\lambda_{\psi}\right\|_{H_{p w}^{\varepsilon}} .
\end{aligned}
$$

The terms involving $\psi-I \psi$ are estimate with the aid of Lemma B.5 and the bound $\|\psi\|_{B_{2, \infty}^{3 / 2}(\Omega)}$ given in (B.7); the terms $\left\|w-w_{h}\right\|_{H^{1}}$ are controlled in (B.3); using $\left\|\lambda_{w}\right\|_{H_{p w}^{\varepsilon}(\Gamma)} \lesssim\|v\|_{H^{-1 / 2+\varepsilon}(\Omega)}$ and (․․7) for $\left\|\lambda_{\psi}\right\|_{H_{p w}^{\varepsilon}(\Gamma)}$ we get

$\left\|\widetilde{\delta}^{-1 / 2}\left(w-w_{h}\right)\right\|_{L^{2}(\Omega)} \lesssim$

$h^{3 / 2}|\ln h|^{1 / 2}\|v\|_{L^{2}(\Omega)}+h^{1}|\ln h|^{1 / 2}\|v\|_{L^{2}(\Omega)} h^{\varepsilon}\|v\|_{H^{-1 / 2+\varepsilon}(\Omega)}+h^{3 / 2} h^{\varepsilon} h^{-\varepsilon}\|v\|_{L^{2}(\Omega)}$.

Now the result follows from (B.5).

Estimating $\mathbf{T}_{\mathbf{2}}$ for $\mathbf{k}>\mathbf{1}$ : We proceed similarly to the case $k=1$. The difference is that (cf. [18, (5.12)]) we need to control

$$
h^{-\varepsilon}\left\|\delta_{\Gamma}^{-1 / 2+\varepsilon}\left(w-w_{h}\right)\right\|_{L^{2}\left(\mathcal{C}^{\prime} \backslash S_{\tilde{c}_{1} h}\right)} .
$$

As in the case $k=1$, we set up a dual problem with solution $\psi=T^{D}\left(\widetilde{\delta}_{\Gamma}^{-1+2 \varepsilon}(w-\right.$ $\left.w_{h}\right)$ ) and corresponding Lagrange multiplier $\lambda_{\psi}$. By Lemma B.4 (and trace estimates) we have the regularity assertions

$$
\left\|\lambda_{\psi}\right\|_{H_{p w}^{\varepsilon}(\Gamma)}+\|\psi\|_{H^{3 / 2+\varepsilon}(\Omega)} \lesssim\left\|\widetilde{\delta}_{\Gamma}^{-1 / 2+\varepsilon}\left(w-w_{h}\right)\right\|_{L^{2}(\Omega)} .
$$


Proceeding in the same manner as in the case $k=1$, we arrive at

$$
\begin{aligned}
& \left\|\widetilde{\delta}^{-1 / 2+\varepsilon}\left(w-w_{h}\right)\right\|_{L^{2}(\Omega)}^{2} \\
& \lesssim\left\|w-w_{h}\right\|_{H^{1}}\|\psi-I \psi\|_{H^{1}}+\|\psi-I \psi\|_{L^{2}}^{1 / 2}\|\psi-I \psi\|_{H^{1}}^{1 / 2} h^{\varepsilon}\left\|\lambda_{w}\right\|_{H_{p w}^{\varepsilon}(\Gamma)} \\
& \quad+h^{1 / 2}\left\|w-w_{h}\right\|_{H^{1}} h^{\varepsilon}\left\|\lambda_{\psi}\right\|_{H_{p w}^{\varepsilon}} .
\end{aligned}
$$

The regularity assertions (B.11) as well as the approximation properties of Lemma B.5 yield $\left\|\widetilde{\delta}^{-1 / 2+\varepsilon}\left(w-w_{h}\right)\right\|_{L^{2}(\Omega)} \lesssim h^{3 / 2+\varepsilon}\|v\|_{L^{2}(\Omega)}$ and hence

$$
h^{-\varepsilon}\left\|\delta_{\Gamma}^{-1 / 2+\varepsilon}\left(w-w_{h}\right)\right\|_{L^{2}\left(\mathcal{C}^{\prime} \backslash S_{\tilde{c}_{1} h}\right)} \lesssim h^{3 / 2}\|v\|_{L^{2}(\Omega)}
$$

Lemma B.4 (Generalization of [18, Lemma 5.2]) Let $\widetilde{\delta}_{\Gamma}$ be the regularized distance function. Then for the operator $T^{D}$ we have for $w:=T^{D}\left(\widetilde{\delta}_{\Gamma}^{-1} v\right)$ (and $\varepsilon>0$ sufficiently small):

$$
\begin{aligned}
\|w\|_{B_{2, \infty}^{3 / 2}(\Omega)} & \leq C|\ln h|^{1 / 2}\left\|\widetilde{\delta}_{\Gamma}^{-1 / 2} v\right\|_{L^{2}(\Omega)}, \\
\|w\|_{H^{3 / 2+\varepsilon}(\Omega)} & \leq C_{\varepsilon} h^{-\varepsilon}\left\|\widetilde{\delta}_{\Gamma}^{-1 / 2} v\right\|_{L^{2}(\Omega)} .
\end{aligned}
$$

For $w=T^{D}\left(\widetilde{\delta}_{\Gamma}^{-1+2 \varepsilon} v\right)$ we have

$$
\|w\|_{H^{3 / 2+\varepsilon}(\Omega)} \leq C\left\|\widetilde{\delta}_{\Gamma}^{-1 / 2+\varepsilon} v\right\|_{L^{2}(\Omega)} .
$$

Proof The proof is done with the same arguments as those of Lemma 2.8

We need an approximation result for the approximation from the constrained space $V_{h}$ for functions that do not permit nodal interpolation.

Lemma B.5 (approximation from constrained space) Let the constrained space $V_{h}$ be defined in [18, (2.4b)] and assume hypotheses [18, (A1), (A2)]. Define the operator $\widetilde{P}_{h}$ as in [18, (4.2)] but replace the interpolation operator $I_{k}$ by a (subdomainwise) Scott-Zhang operator $I_{h}^{k, S Z}$ (cf. [20]) that conforms to the boundaries of the subdomains $\Omega_{i}, i=1, \ldots, M$. Then $\widetilde{P}_{h}$ is defined on $H^{s}(\Omega) \cap H_{0}^{1}(\Omega)$ for $s \geq 1$, it maps into $V_{h}$, and has the approximation properties

$$
\begin{aligned}
\sqrt{\sum_{i}\left\|v-\widetilde{P}_{h} v\right\|_{H^{1}\left(\Omega_{i}\right)}^{2}} & \leq C h^{s-1}\|v\|_{H^{s}(\Omega)}, \\
\left\|v-\widetilde{P}_{h} v\right\|_{L^{2}(\Omega)} & \leq C h^{s}\|v\|_{H^{s}(\Omega)}, \quad 1 \leq s \leq k+1, \\
\sqrt{\sum_{i}\left\|v-\widetilde{P}_{h} v\right\|_{H^{1}\left(\Omega_{i}\right)}^{2}} & \leq C h^{s-1}\|v\|_{B_{2, \infty}^{s}(\Omega)}, \\
\left\|v-\widetilde{P}_{h} v\right\|_{L^{2}(\Omega)} & \leq C h^{s}\|v\|_{B_{2, \infty}^{s}(\Omega)}, \quad 1<s<k+1, \quad s \notin \mathbb{N} .
\end{aligned}
$$


Proof We only show the first estimates as the estimates with $B_{2, \infty}^{s}(\Omega)$-regularity follow by interpolation arguments. It suffices to study the contribution $E_{k} \Pi_{h}\left[I^{k, S Z} v\right]$ to the operator $\widetilde{P}_{h}$. We recall that by the multiplicative trace inequality $\|w\|_{L^{2}\left(\partial \Omega_{i}\right)}^{2} \lesssim$ $\|w\|_{L^{2}\left(\Omega_{i}\right)}\|w\|_{H^{1}\left(\Omega_{i}\right)}$ and the simultaneous approximation properties of $I^{k, S Z}$ in $L^{2}$ and $H^{1}$ we have $\left\|v-I^{k, S Z} v\right\|_{L^{2}\left(\partial \Omega_{i}\right)} \leq C h^{s-1 / 2}\|v\|_{H^{s}\left(\Omega_{i}\right)}$. Exploiting the $L^{2}$-stability of the mortar projection $\Pi_{h}$, we get the bound

$$
\begin{aligned}
\left\|E_{k} \Pi_{h}\left[I^{k, S Z} v\right]\right\|_{H^{1}\left(\Omega_{i}\right)} & \lesssim h^{-1} h^{1 / 2}\left\|\left[I^{k, S Z} v\right]\right\|_{L^{2}\left(\partial \Omega_{i}\right)} \\
& \lesssim h^{-1 / 2}\left\|v-I^{k, S Z} v\right\|_{L^{2}\left(\partial \Omega_{i}\right)} \lesssim h^{s-1}\|v\|_{H^{s}\left(\Omega_{i}\right)} .
\end{aligned}
$$




\section{Details concerning Lemma 2.9}

The following lemma is an expanded version of Lemma 2.9 It is closely related to Lemma 2.3 with $\beta=1 / 2$ there. The essential difference is that we replace the norm $\|z\|_{H^{3 / 2}(\Omega)}$ with the weaker norm $\|z\|_{B_{2, \infty}^{3 / 2}(\Omega)}$ at the expense of a factor $|\ln h|^{1 / 2}$.

Lemma C.1 Let the bounded Lipschitz domain $\Omega \subset \mathbb{R}^{d}, d \in\{2,3\}$. Assume that $w \in B_{2, \infty}^{3 / 2}(\Omega)$ is a solution of

$$
-\nabla \cdot(\mathbf{A} \nabla w)=v
$$

(i) There exist constants $C$ (depending only on the lower bound $\alpha_{0}$ for the eigenvalues of $\mathbf{A}$, the norm $\|\mathbf{A}\|_{C^{0,1}(\bar{\Omega})}$, and $\Omega$ ) and $c_{1}>0$ (depending only on $\Omega$ ) such that with the distance function $\delta_{\Gamma}$

$$
\left\|\sqrt{\delta_{\Gamma}} \nabla^{2} w\right\|_{L^{2}\left(\Omega \backslash S_{h}\right)} \leq C \sqrt{|\ln h|}\|w\|_{B_{2, \infty}^{3 / 2}(\Omega)}+C\left\|\sqrt{\delta_{\Gamma}} v\right\|_{L^{2}\left(\Omega \backslash S_{c_{1} h}\right)} .
$$

In particular, if $\left.v\right|_{\Omega \backslash S_{c_{1} h}}=0$ then $\left\|\sqrt{\delta_{\Gamma}} \nabla^{2} w\right\|_{L^{2}\left(\Omega \backslash S_{h}\right)} \leq C \sqrt{|\ln h|}\|w\|_{B_{2, \infty}^{3 / 2}(\Omega)}$.

(ii) Let $c^{\prime}>0$ be fixed. Assume $\left.v\right|_{\Omega \backslash S_{c^{\prime} h}}=0$. Then there exist $\widetilde{c}, c_{2}>0$ (depending only on $\Omega$ ) such for every $\alpha>0$

$$
\begin{aligned}
\left\|\delta_{\Gamma}^{\alpha} \nabla^{3} w\right\|_{L^{2}\left(\Omega \backslash S_{\tilde{c} h}\right)} \leq & C_{A, \Omega}\left[\left\|\delta_{\Gamma}^{\alpha-1} \nabla^{2} w\right\|_{L^{2}\left(\Omega \backslash S_{c_{2} h}\right)}\right. \\
& \left.+\|\mathbf{A}\|_{C^{1,1}(\Omega)}\left\|\delta_{\Gamma}^{\alpha} \nabla w\right\|_{L^{2}\left(\Omega \backslash S_{c_{2} h}\right)}\right]
\end{aligned}
$$

the constant $C_{A, \Omega}$ depends on the coercivity constant $\alpha_{0}$ and $\|\mathbf{A}\|_{C^{0,1}(\bar{\Omega})}$ as well as $\Omega$.

(iii) Let $c^{\prime}>0$ be fixed. Assume $\left.v\right|_{\Omega \backslash S_{c^{\prime} h}}=0$. Assume that $w \in H^{3 / 2+\varepsilon}(\Omega)$ for some $\varepsilon \in(0,1 / 2)$. Then there exists $\widehat{c}>0$ (depending only on $\Omega$ and $c^{\prime}$ ) such that

$$
\left\|\nabla^{2} w\right\|_{L^{2}\left(\Omega \backslash S_{\overparen{c} h}\right)} \leq C_{A, \varepsilon} h^{-1 / 2+\varepsilon}\|w\|_{H^{3 / 2+\varepsilon}(\Omega)},
$$

where $C_{A, \varepsilon}$ depends on $\alpha_{0},\|\mathbf{A}\|_{C^{0,1}(\bar{\Omega})}$, and $\varepsilon$.

Proof

Proof of (2): We may restrict our attention to a local situation near a part of the boundary. The boundary $\Gamma$ can locally be described by a graph $\phi$. That is, in a suitable coordinate system, we can define cylinders

$$
\begin{aligned}
& \mathcal{C}_{\delta}=\{(x, \phi(x)+t) \mid \delta<t<D+\delta, x \in B\}, \\
& \mathcal{C}_{\delta}^{\prime}=\left\{(x, \phi(x)+t) \mid \delta<t<D^{\prime}+\delta, x \in B^{\prime}\right\},
\end{aligned}
$$

where $\delta<D<D^{\prime}$ and $B, B^{\prime}$ are two concentric balls with $B \subset \subset B^{\prime}$. Furthermore, for $\delta=0$ we assume $\mathcal{C}_{0}^{\prime} \subset \Omega$. In particular, $\left\{(x, \phi(x)) \mid x \in B^{\prime}\right\} \subset \Gamma$. We also note that $t \sim \operatorname{dist}((x, \phi(x)+t), \Gamma)$.

Let $\mathcal{C}_{\delta}^{\prime \prime}$ be a third cylinder of the form $\mathcal{C}_{\delta}^{\prime \prime}=\left\{(x, \phi(x)+t): \delta<t<D^{\prime \prime}+\delta, x \in\right.$ $\left.B^{\prime \prime}\right\}$ where $B \subset \subset B^{\prime \prime} \subset \subset B^{\prime}$ and $D<D^{\prime \prime}<D^{\prime}$. Let $\chi \in C^{\infty}\left(\mathbb{R}^{d}\right)$ be such that $\left.\chi\right|_{\mathcal{C}_{0}^{\prime \prime}} \equiv 1$ and $\left.\chi\right|_{\Omega \backslash \mathcal{C}_{0}^{\prime}} \equiv 0$. To simplify the notation, we assume that the functions $w, \mathbf{A}, v$ are given in a coordinate system commensurate with the coordinate system describing the cylinders $\mathcal{C}_{\delta}, \mathcal{C}_{\delta}^{\prime}$, viz., $w$ evaluated at a point $(x, \phi(x)+t) \in \mathcal{C}_{\delta}^{\prime}$ is 
given by $w(x, \phi(x)+t)$. A translation in the last variable defines the function $\widetilde{w}$ by $\widetilde{w}(x, \phi(x)+t):=w(x, \phi(x)+t+2 \delta)$. We note

$$
-\nabla \cdot(\tilde{\mathbf{A}} \nabla \tilde{w})=\widetilde{v} \quad \text { in } \mathcal{C}_{-2 \delta}^{\prime}
$$

here (again in the coordinate system used to describe the cylinders) $\widetilde{\mathbf{A}}(x, \phi(x)+$ $t)=\mathbf{A}(x, \phi(x)+t+2 \delta)$ and $\widetilde{v}(x, \phi(x)+t)=v(x, \phi(x)+t+2 \delta)$.

1. step: We show (if $\delta$ is sufficiently small)

$$
\|\widetilde{w}\|_{H^{3 / 2}\left(\mathcal{C}_{0}^{\prime \prime}\right)} \leq C_{A, \Omega}\left[\sqrt{|\ln \delta|}\|w\|_{B_{2, \infty}^{3 / 2}(\Omega)}+\delta\|\tilde{v}\|_{L^{2}\left(\mathcal{C}_{-\delta}^{\prime}\right)}\right],
$$

where $C_{A, \Omega}$ depends on $\Omega$, the coercivity constant $\alpha_{0}$ and $\|\mathbf{A}\|_{\mathcal{C}^{0,1}(\bar{\Omega})}$.

Using the characterization of $H^{3 / 2}\left(\mathcal{C}_{0}^{\prime}\right)=\left(H^{1}\left(\mathcal{C}_{0}^{\prime}\right), H^{2}\left(\mathcal{C}_{0}^{\prime}\right)\right)_{1 / 2,2}$ in terms of the $K$-functional, we write (cf. also [5, p.193, eqn. (7.4)])

$$
\begin{aligned}
\|\chi \widetilde{w}\|_{H^{3 / 2}\left(\mathcal{C}_{0}^{\prime}\right)}^{2} & =\int_{t=0}^{1}\left(t^{-1 / 2} K(t, \chi \widetilde{w})\right)^{2} \frac{d t}{t} \\
& =\int_{t=0}^{\varepsilon}\left(t^{-1 / 2} K(t, \chi \widetilde{w})\right)^{2} \frac{d t}{t}+\int_{t=\varepsilon}^{1}\left(t^{-1 / 2} K(t, \chi \widetilde{w})\right)^{2} \frac{d t}{t} .(\mathrm{C} .5)
\end{aligned}
$$

The second integral in (C.5) can be estimated by

$$
\int_{t=\varepsilon}^{1}\left(t^{-1 / 2} K(t, \chi \widetilde{w})\right)^{2} \frac{d t}{t} \leq \int_{t=\varepsilon}^{1} \frac{d t}{t} \sup _{t>0}\left(t^{-1 / 2} K(t, \chi \widetilde{w})\right)^{2} \leq \ln \varepsilon\|\chi \widetilde{w}\|_{B_{2, \infty}^{3 / 2}\left(\mathcal{C}_{0}^{\prime}\right)}^{2} .
$$

For the first integral in (C.5) we employ interior regularity estimates for solutions of second order elliptic equation with vanishing right-hand side. Specifically, C.3 and interior regularity (see, e.g., [9, Thm. 8.8 and proof]) give (here, we assume that $\delta$ is sufficiently small)

$$
\|\chi \widetilde{w}\|_{H^{2}\left(\mathcal{C}_{0}^{\prime}\right)} \leq C_{A}\left[\delta^{-1}\|\widetilde{w}\|_{H^{1}\left(\mathcal{C}_{-\delta}^{\prime}\right)}+\|\widetilde{v}\|_{L^{2}\left(\mathcal{C}_{-\delta}^{\prime}\right)}\right],
$$

where the constant $C_{A}$ depends only on the coercivity constant $\alpha_{0}$ and $\|\mathbf{A}\|_{C^{0,1}(\bar{\Omega})}$.

Hence, estimating $K(t, \chi \widetilde{w})=\inf _{v \in H^{2}}\|\chi \widetilde{w}-v\|_{H^{1}\left(\mathcal{C}_{0}^{\prime}\right)}+t\|v\|_{H^{2}\left(\mathcal{C}_{0}^{\prime}\right)} \leq t\|\chi \widetilde{w}\|_{H^{2}\left(\mathcal{C}_{0}^{\prime}\right)}$, we obtain

$$
\int_{t=0}^{\varepsilon} t^{-2} K^{2}(t, \chi \widetilde{w}) d t \leq \varepsilon\|\chi \widetilde{w}\|_{H^{2}\left(\mathcal{C}_{0}^{\prime}\right)}^{2} \leq C_{A}\left[\varepsilon \delta^{-2}\|\widetilde{w}\|_{H^{1}\left(\mathcal{C}_{-\delta}^{\prime}\right)}^{2}+\varepsilon\|\widetilde{v}\|_{L^{2}\left(\mathcal{C}_{-\delta}^{\prime}\right)}^{2}\right] .
$$

We conclude

$$
\begin{aligned}
\|\chi \widetilde{w}\|_{H^{3 / 2}\left(\mathcal{C}_{0}^{\prime}\right)}^{2} & \leq C\left[\varepsilon \delta^{-2}\|\widetilde{w}\|_{H^{1}\left(\mathcal{C}_{-\delta}^{\prime}\right)}^{2}+\varepsilon\|\widetilde{v}\|_{L^{2}\left(\mathcal{C}_{-\delta}^{\prime}\right)}^{2}+\ln \varepsilon\|\chi \widetilde{w}\|_{B_{2, \infty}^{3 / 2}\left(\mathcal{C}_{0}^{\prime}\right)}^{2}\right] \\
& \leq C_{A, \Omega}\left\{\left[\varepsilon \delta^{-2}+\ln \varepsilon\right]\|w\|_{B_{2, \infty}^{3 / 2}\left(\mathcal{C}_{0}^{\prime}\right)}^{2}+\varepsilon\|\widetilde{v}\|_{L^{2}\left(\mathcal{C}_{-\delta}^{\prime}\right)}^{2}\right\},
\end{aligned}
$$

where, in the last step we have employed that multiplication by a smooth function and translation are bounded operations on Sobolev (and therefore also Besov) spaces; the constant $C_{A, \Omega}$ depends on $\Omega, \alpha_{0}$, and $\|\mathbf{A}\|_{C^{0,1}(\bar{\Omega})}$. Selecting $\varepsilon=\delta^{2}$ shows $\|\chi \widetilde{w}\|_{H^{3 / 2}\left(\mathcal{C}_{0}^{\prime}\right)} \leq C_{A, \Omega}\left[\sqrt{|\ln \delta|}\|w\|_{B_{2, \infty}^{3 / 2}(\Omega)}+\delta\|\widetilde{v}\|_{L^{2}\left(\mathcal{C}_{-\delta}^{\prime}\right)}\right]$ from which we get (C.4) in view of the support properties of $\chi$. 
Step 2: Let $z$ solve, for a fixed $\rho>0$ and a parameter $r \leq 1$, the equation

$$
-\nabla \cdot(\widetilde{\mathbf{A}} \nabla z)=f \quad \text { in a ball } B_{r(1+\rho)} \text { of radius } r(1+\rho) .
$$

We claim: For a constant $C_{A, \rho}>0$ that depends solely on the coercivity constant $\alpha_{0},\|\mathbf{A}\|_{C^{0,1}(\bar{\Omega})}$, and $\rho$ there holds

$$
\begin{aligned}
\left\|\nabla^{2} z\right\|_{L^{2}\left(B_{r}\right)} & \leq C_{A, \rho}\left[\|f\|_{L^{2}\left(B_{r(1+\rho)}\right)}+\|\nabla z\|_{L^{2}\left(B_{r(1+\rho)}\right)}+r^{-1 / 2}|z|_{H^{3 / 2}\left(B_{r(1+\rho)}\right)}\right] \\
\left\|\nabla^{2} z\right\|_{L^{2}\left(B_{r}\right)} & \leq C_{A, \rho}\left[\|f\|_{L^{2}\left(B_{r(1+\rho)}\right)}+r^{-1}\|\nabla z\|_{L^{2}\left(B_{r(1+\rho)}\right)}\right] .
\end{aligned}
$$

We point out that the $H^{3 / 2}$-seminorm in (C.7) is defined in terms of the AronsteinSlobodeckij norm for scaling reasons. The bounds (C.7), (C.8) follow from interior regularity in the following way.

Scaling the ball $B_{r(1+\rho)}$ to a ball $\widehat{B}_{1+\rho}$ of radius $1+\rho$ leads to an equation of the form

$$
-\nabla \cdot(\widehat{\mathbf{A}} \nabla \widehat{z})=r^{2} \widehat{f} \quad \text { in a ball } \widehat{B}_{1+\rho} \text { of radius }(1+\rho),
$$

where $\widehat{\mathbf{A}}$ and $\widehat{f}$ are the coefficient and the right-hand side in the scaled variables. We note that

$$
\left\|\nabla^{j} \widehat{\mathbf{A}}\right\|_{L^{\infty}\left(\widehat{B}_{1+\rho}\right)} \sim r^{j}\left\|\nabla^{j} \widetilde{\mathbf{A}}\right\|_{L^{\infty}\left(B_{r(1+\rho)}\right)}, \quad j \in \mathbb{N}_{0} .
$$

Then standard interior regularity (see, e.g., [9, Thm. 8.8]) gives

$$
\left\|\nabla^{2} \widehat{z}\right\|_{L^{2}\left(\widehat{B}_{1}\right)} \leq C_{A, \rho}\left[r^{2}\|\widehat{f}\|_{L^{2}\left(\widehat{B}_{1+\rho}\right)}+\|\widehat{z}\|_{H^{1}\left(\widehat{B}_{1+\rho}\right)}\right]
$$

where the constant $C_{A, \rho}$ depends only on the coercivitiy constant of $\widehat{\mathbf{A}}$, the norm $\|\widehat{\mathbf{A}}\|_{C^{0,1}\left(B_{1+\rho}\right)}$, and $\rho$. In view of $r \leq 1$ and the fact that the operator $\widetilde{\mathbf{A}}$ is obtained from $\mathbf{A}$ by an affine change of variables (a translation and an orthogonal transformation) we bound $\|\widehat{\mathbf{A}}\|_{C^{0,1}\left(\widehat{B}_{1+\rho}\right)} \leq C\|A\|_{C^{0,1}(\bar{\Omega})}$ and get

$$
\left\|\nabla^{2} \widehat{z}\right\|_{L^{2}\left(\widehat{B}_{1}\right)} \leq C_{A, \rho}\left[r^{2}\|\widehat{f}\|_{L^{2}\left(\widehat{B}_{1+\rho}\right)}+\|\widehat{z}\|_{H^{1}\left(\widehat{B}_{1+\rho}\right)}\right]
$$

for a constant $C_{A, \rho}$ that depends only on $\rho$, the coercivity constant of $\mathbf{A}$, and $\|\mathbf{A}\|_{C^{0,1}(\bar{\Omega})}$. Since the constant functions are in the kernel of the operator $-\nabla$. $(\widehat{\mathbf{A}} \nabla z)$ it is easy to conclude with a Poincaré inequality that (C.11) implies

$$
\left\|\nabla^{2} \widehat{z}\right\|_{L^{2}\left(\widehat{B}_{1}\right)} \leq C_{A, \rho}\left[r^{2}\|\widehat{f}\|_{L^{2}\left(\widehat{B}_{1+\rho}\right)}+\|\nabla \widehat{z}\|_{L^{2}\left(\widehat{B}_{1+\rho}\right)}\right]
$$

Scaling this equation back to $B_{r(1+\rho)}$ yields the desired bound (C.8). For the proof of (C.7), we have to bring in the $H^{3 / 2}$-seminorm. Let $\pi \in \mathcal{P}_{1}$ be arbitrary. Then the function $\widehat{z}-\pi$ satisfies in view of the fact that $\nabla \pi$ is constant

$$
-\nabla \cdot(\widehat{\mathbf{A}} \nabla(\widehat{z}-\pi))=r^{2} \widehat{f}+\nabla \cdot(\widehat{\mathbf{A}} \nabla \pi)=r^{2} \widehat{f}+(\nabla \cdot \widehat{\mathbf{A}}) \cdot \nabla \pi=: \widetilde{f} .
$$


(We employed the convention that the divergence operator $\nabla \cdot$ in the expression $\nabla \cdot \widehat{\mathbf{A}}$ acts on columns of $\widehat{\mathbf{A}}$ ). Applying (C.11) to this equation (and replacing $r^{2} \widehat{f}$ with $\tilde{f}$ and $\widehat{z}$ with $\widehat{z}-\pi$ ) yields

$$
\begin{aligned}
\left\|\nabla^{2} \widehat{z}\right\|_{L^{2}\left(\widehat{B}_{1}\right)} & \leq\|\widehat{z}-\pi\|_{H^{2}\left(\widehat{B}_{1}\right)} \leq C_{A, \rho}\left[\|\widetilde{f}\|_{L^{2}\left(\widehat{B}_{1+\rho}\right)}+\|\widehat{z}-\pi\|_{H^{1}\left(\widehat{B}_{1+\rho}\right)}\right] \\
& \leq C_{A, \rho}\left[r^{2}\|\widehat{f}\|_{L^{2}\left(B_{r(1+\rho)}\right)}+r\|\nabla \pi\|_{L^{2}\left(\widehat{B}_{1+\rho}\right)}+\|\widehat{z}-\pi\|_{H^{3 / 2}\left(\widehat{B}_{1+\rho}\right)}\right] \\
& \leq C_{A, \rho}\left[r^{2}\|\widehat{f}\|_{L^{2}\left(B_{r(1+\rho)}\right)}+r\|\nabla \widehat{z}\|_{L^{2}\left(\widehat{B}_{1+\rho}\right)}+\|\widehat{z}-\pi\|_{H^{3 / 2}\left(\widehat{B}_{1+\rho}\right)}\right] .
\end{aligned}
$$

Infimizing over all $\pi \in \mathcal{P}_{1}$ yields

$$
\left\|\nabla^{2} \widehat{z}\right\|_{L^{2}\left(\widehat{B}_{1}\right)} \leq C_{A, \rho}\left[r^{2}\|\widehat{f}\|_{L^{2}\left(\widehat{B}_{1+\rho}\right)}+r\|\nabla \widehat{z}\|_{L^{2}\left(\widehat{B}_{1+\rho}\right)}+|\widehat{z}|_{H^{3 / 2}\left(\widehat{B}_{1+\rho}\right)}\right]
$$

Scaling back to $B_{r(1+\rho)}$ yields (C.7), if we note the scaling properties of the Aronstein-Slobodeckij seminorm.

3. step: Applying the result of step 2 to the function $\widetilde{w}$ yields

$$
\left\|\nabla^{2} \widetilde{w}\right\|_{L^{2}\left(B_{r}\right)} \leq C_{A, \rho}\left[\|\nabla \widetilde{w}\|_{L^{2}\left(B_{r(1+\rho)}\right)}+r^{-1 / 2}|\widetilde{w}|_{H^{3 / 2}\left(B_{(1+\rho) r}\right)}+\|\widetilde{v}\|_{L^{2}\left(B_{r(1+\rho)}\right)}\right]
$$

for all balls $B_{r}$ such that $B_{(1+\rho) r} \subset \mathcal{C}_{-2 \delta}^{\prime}$. Using, for example, the Besicovitch covering theorem [7], we can cover $\mathcal{C}_{0}$ by overlapping balls $B_{r_{i}}\left(x_{i}\right)$ with centers $x_{i}$ and radii $r_{i} \sim \delta_{\Gamma}\left(x_{i}\right)$ such that the stretched balls $B_{r_{i}(1+\rho)}\left(x_{i}\right)$ have a finite overlap property (see [18, Lemma A.1] for details). A covering argument and afterwards (C.4) then show

$$
\begin{aligned}
\left\|\sqrt{\delta_{\Gamma}} \nabla^{2} \widetilde{w}\right\|_{L^{2}\left(\mathcal{C}_{0}\right)} & \leq C_{A, \Omega}\left[\|\widetilde{w}\|_{H^{3 / 2}\left(\mathcal{C}_{0}^{\prime \prime}\right)}+\left\|\sqrt{\delta_{\Gamma}} \nabla \widetilde{w}\right\|_{L^{2}\left(\mathcal{C}_{0}^{\prime}\right)}+\left\|\sqrt{\delta_{\Gamma}} \widetilde{v}\right\|_{L^{2}\left(\mathcal{C}_{0}^{\prime}\right)}\right] \\
& \leq C_{A, \Omega}\left[\sqrt{|\ln \delta|}\|w\|_{B_{2, \infty}^{3 / 2}(\Omega)}+\delta\|\widetilde{v}\|_{L^{2}\left(\mathcal{C}_{-\delta}^{\prime}\right)}+\left\|\sqrt{\delta_{\Gamma}} \widetilde{v}\right\|_{L^{2}\left(\mathcal{C}_{0}^{\prime}\right)}\right]
\end{aligned}
$$

where the constant $C_{A, \Omega}$ depends only on the coercivity constant $\alpha_{0},\|\mathbf{A}\|_{C^{0,1}(\bar{\Omega})}$, and $\Omega$.

Since $\widetilde{w}$ and $\widetilde{v}$ are obtained by a translation, we arrive at

$$
\left\|\sqrt{\delta_{\Gamma}} \nabla^{2} w\right\|_{L^{2}\left(\mathcal{C}_{2 \delta}\right)} \leq C_{A, \Omega}\left[\sqrt{|\ln \delta|}\|w\|_{B_{2, \infty}^{3 / 2}(\Omega)}+\left\|\sqrt{\delta_{\Gamma}} v\right\|_{L^{2}\left(\Omega \backslash S_{c_{2} \delta}\right)}\right]
$$

for a suitable $c_{2}$ that depends solely on the Lipschitz character of $\Gamma$. Taking $\delta \sim h$ produces the desired result.

Proof of (ii): The estimate merely expresses interior regularity for solutions of elliptic equations with vanishing right-hand side. It follows from (C.8) and a covering argument. More precisely, as in Step 2, we start from

$$
-\nabla \cdot(\mathbf{A} \nabla z)=f \quad \text { in a ball } B_{r(1+\rho)} \text { of radius } r(1+\rho) .
$$

Differentiating this equation once gives for $\alpha \in \mathbb{N}^{d}$ with $|\alpha|=1$

$-\nabla \cdot\left(\mathbf{A} \nabla D^{\alpha} z\right)=D^{\alpha} f+\nabla \cdot\left(D^{\alpha} \mathbf{A}\right) \nabla z \quad$ in a ball $B_{r(1+\rho)}$ of radius $r(1+\rho)$ 
where again the divergence operator in the expression $\nabla \cdot\left(D^{\alpha} \mathbf{A}\right)$ acts on the columns of $D^{\alpha} \mathbf{A}$. We get from (C.8) by considering all $\alpha \in \mathbb{N}^{d}$ with $|\alpha|=1$

$\left\|\nabla^{3} z\right\|_{L^{2}\left(B_{r}\right)} \leq$

$$
C_{A, \rho}\left[\|\nabla f\|_{L^{2}\left(B_{r(1+\rho)}\right)}+r^{-1}\left\|\nabla^{2} z\right\|_{L^{2}\left(B_{r(1+\rho)}\right)}+\left\|\nabla^{2} \mathbf{A}\right\|_{L^{\infty}\left(B_{r(1+\rho)}\right)}\|\nabla z\|_{L^{2}\left(B_{r(1+\rho)}\right)}\right] .
$$

A covering argument then produces the claim since $f$ is assumed to satisfy $f \equiv 0$ on $\Omega \backslash S_{c^{\prime} h}$ in the statement (iii). We also note that $\left\|\nabla^{2} \mathbf{A}\right\|_{L^{\infty}(\Omega)}$ can be bounded by $\|\nabla \mathbf{A}\|_{W^{1, \infty}(\Omega)}$.

Proof of (iii): Our starting point is the above Step 2: We claim that for the function $z$ satisfying (C.9) we have

$\left\|\nabla^{2} z\right\|_{L^{2}\left(B_{r}\right)} \leq C_{A}\left[\|f\|_{L^{2}\left(B_{r(1+\rho)}\right)}+\|\nabla z\|_{L^{2}\left(B_{r(1+\rho)}\right)}+r^{-1 / 2+\varepsilon}|z|_{H^{3 / 2+\varepsilon}\left(B_{r(1+\rho)}\right)}\right]$,

where the $H^{3 / 2+\varepsilon}$-seminorm is again an Aronstein-Slobodeckij norm, and the constant $C_{A}$ depends only on the coercivity constant $\alpha_{0},\|\mathbf{A}\|_{C^{0,1}(\bar{\Omega})}$, and $\varepsilon \in(0,1 / 2)$. To see this, we check the derivation of (C.13). We see that one can (marginally) modify the arguments to obtain instead of (C.13) the estimate

$$
\left\|\nabla^{2} \widehat{z}\right\|_{L^{2}\left(\widehat{B}_{1+\rho}\right)} \lesssim r^{2}\|\widehat{f}\|_{L^{2}\left(\widehat{B}_{1+\rho}\right)}+r\|\nabla \widehat{z}\|_{L^{2}\left(\widehat{B}_{1+\rho}\right)}+|\widehat{z}|_{H^{3 / 2+\varepsilon}\left(\widehat{B}_{1+\rho}\right)},
$$

where the implied constant depends on $\rho, \alpha_{0},\|\mathbf{A}\|_{C^{0,1}(\bar{\Omega})}$, and $\varepsilon$. Scaling back to $B_{r(1+\rho)}$ yields

$r^{2}\left\|\nabla^{2} z\right\|_{L^{2}\left(B_{r(1+\rho)}\right)} \lesssim r^{2}\|f\|_{L^{2}\left(B_{r(1+\rho)}\right)}+r r\|\nabla z\|_{L^{2}\left(B_{r(1+\rho)}\right)}+r^{3 / 2+\varepsilon}|z|_{H^{3 / 2+\varepsilon}\left(\widehat{B}_{r(1+\rho)}\right)}$,

which leads to C.15). We now use $f \equiv 0$ on $\Omega \backslash S_{C^{\prime} h}$ and use these estimates with $w$ in place of $z$. A covering argument then gives

$$
\left\|\nabla^{2} w\right\|_{L^{2}\left(\Omega \backslash S_{\overparen{c} h}\right)} \lesssim\|\nabla w\|_{L^{2}\left(\Omega \backslash S_{c^{\prime} h}\right)}+h^{-1 / 2+\varepsilon}\|w\|_{H^{3 / 2+\varepsilon}(\Omega)} .
$$

This concludes the proof of (iii). 


\section{Details of [24, Sec. 5.3]}

By and large, we follow the arguments of [24, Sec. 5.3] and adapt them as needed.

In what follows, $\mathcal{T}$ is a quasi-uniform mesh with mesh size $h$. We consider the bilinear form

$$
a(u, v):=\int_{\Omega} \mathbf{A} \nabla u \cdot \nabla v
$$

where the matrix $\mathbf{A}$ is sufficiently smooth and pointwise symmetric positive definite.

\section{D.1 The interior case}

In this subsection, we assume that $V_{h}=S^{k, 1}(\mathcal{T})$.

For balls $B_{d}$ of radius $d$, it will be convenient to introduce the notation

$$
\|u\|_{1, B_{d}}:=|u|_{H^{1}\left(B_{d}\right)}+\frac{1}{d}\|u\|_{L^{2}\left(B_{d}\right)} .
$$

We start with making the notion of "superapproximation" more precise:

Lemma D.1 (superapproximation on balls) Let $B_{d} \subset \Omega$ be a ball of radius d. Let $\omega \in C^{\infty}$ with $\operatorname{supp} \omega \subset B_{d / 2}$ and

$$
\left\|\nabla^{j} \omega\right\|_{L^{\infty}} \leq C d^{-j}, \quad j=0, \ldots, k .
$$

Then for every $u \in V_{h}$ the interpolant $I\left(\omega^{2} u\right) \in S^{k, 1}(\mathcal{T}) \cap H_{0}^{1}\left(B_{d}\right)$ satisfies

$$
\begin{aligned}
\left|\omega^{2} u-I\left(\omega^{2} u\right)\right|_{H^{1}\left(B_{d}\right)} & \leq C \frac{h}{d}\|u\|_{1, B_{d}}, \\
\frac{1}{d}\left\|\omega^{2} u-I\left(\omega^{2} u\right)\right\|_{L^{2}\left(B_{d}\right)} & \leq C\left(\frac{h}{d}\right)^{2}\|u\|_{1, B_{d}} .
\end{aligned}
$$

We assume implicitly that $d>h$ is sufficiently large.

Proof Since supp $\omega \subset B_{d / 2}$ and $d$ is large compared to $h$, we have supp $I\left(\omega^{2} u\right) \subset$ $B_{d}$. For each element $K$ we have the estimates

$$
\left\|\omega^{2} u-I\left(\omega^{2} u\right)\right\|_{L^{2}(K)}+h\left\|\nabla\left(\omega^{2} u-I\left(\omega^{2} u\right)\right)\right\|_{L^{2}(K)} \leq C h^{k+1}\left\|\nabla^{k+1}\left(\omega^{2} u\right)\right\|_{L^{2}(K)} .
$$

Inductively, we see that $\left\|\nabla^{j}\left(\omega^{2}\right)\right\|_{L^{\infty}} \leq C d^{-j}$ for $j=0, \ldots, k$. Using the fact that $u$ is piecewise polynomial of degree $k$ we conclude

$$
\|\left(\nabla^{k+1}\left(\omega^{2} u\right)\left\|_{L^{2}(K)} \leq C \sum_{j=0}^{k} d^{-(k+1-j)}\right\| \nabla^{j} u \|_{L^{2}(K)} .\right.
$$

An inverse estimate produces in view of $h / d \lesssim 1$

$$
\begin{aligned}
\left\|\omega^{2} u-I\left(\omega^{2} u\right)\right\|_{L^{2}(K)}+h\left\|\nabla\left(\omega^{2} u-I\left(\omega^{2} u\right)\right)\right\|_{L^{2}(K)} & \leq C h \frac{h}{d}\left[\frac{1}{d}\|u\|_{L^{2}\left(B_{d}\right)}+|u|_{H^{1}\left(B_{d}\right)}\right] \\
& \leq C h \frac{h}{d}\|u\|_{1, B_{d}} .
\end{aligned}
$$


The next result shows an inverse estimate for "discrete harmonic" functions:

Lemma D.2 Let $u_{h} \in V_{h}$ satisfy

$$
a\left(u_{h}, v\right)=0 \quad \forall v \in V_{h} \quad \text { with } \quad \operatorname{supp} v \subset B_{d}
$$

for a ball $B_{d} \subset \Omega$ of radius d (implicitly assumed sufficiently large compared to h). Then

$$
\left\|\nabla u_{h}\right\|_{L^{2}\left(B_{d / 2}\right)} \leq C d^{-1}\left\|u_{h}\right\|_{L^{2}\left(B_{d}\right)} .
$$

Proof Select a cut-off function $\omega$ with $\operatorname{supp} \omega \subset B_{d}$ and D.1 as well as $\chi \equiv 1$ on $B_{d / 2}$.

We write for arbitrary $\chi \in V_{h}$ with $\operatorname{supp} \chi \subset B_{d}$

$$
\begin{aligned}
\int_{\Omega} \omega^{2} \mathbf{A} \nabla u_{h} \cdot \nabla u_{h} & =\int_{\Omega} \mathbf{A} \nabla u_{h} \cdot \nabla\left(\omega^{2} u_{h}\right)-\int_{\Omega} 2 u_{h} \omega \nabla \omega \cdot \mathbf{A} \nabla u_{h} \\
& =\int_{\Omega} \mathbf{A} \nabla u_{h} \cdot \nabla\left(\omega^{2} u_{h}-\chi\right)-\int_{\Omega} 2 u_{h} \omega \nabla \omega \cdot \mathbf{A} \nabla u_{h} .
\end{aligned}
$$

We conclude from Lemma D.1 with $\chi=I\left(\omega^{2} u_{h}\right)$ there and Young's inequality

$$
\int_{\Omega} \omega^{2} \mathbf{A} \nabla u_{h} \cdot \nabla u_{h} \lesssim \frac{h}{d}\left\|\nabla u_{h}\right\|_{L^{2}\left(B_{d}\right)}\left\|u_{h}\right\|_{1, B_{d}}+\frac{1}{d^{2}}\left\|u_{h}\right\|_{L^{2}\left(B_{d}\right)}^{2} .
$$

We conclude

$$
\left\|\nabla u_{h}\right\|_{L^{2}\left(B_{d / 2}\right)} \lesssim \sqrt{\frac{h}{d}}\left\|u_{h}\right\|_{1, B_{d}}+\frac{1}{d}\left\|u_{h}\right\|_{L^{2}\left(B_{d}\right)}
$$

Iterating the argument yields

$$
\left\|\nabla u_{h}\right\|_{L^{2}\left(B_{d / 4}\right)} \lesssim \frac{h}{d}\left\|u_{h}\right\|_{1, B_{d}}+\frac{1}{d}\left\|u_{h}\right\|_{L^{2}\left(B_{d}\right)} .
$$

Finally, a standard inverse estimate produces

$$
\left\|\nabla u_{h}\right\|_{L^{2}\left(B_{d / 4}\right)} \lesssim \frac{1}{d}\left\|u_{h}\right\|_{L^{2}\left(B_{d}\right)},
$$

which is the desired final bound.

We now show the main result:

Theorem D.3 Let $u \in H^{1}(\Omega)$ and $u_{h} \in V_{h}$ be such that

$$
a\left(u-u_{h}, v\right)=0 \quad \forall v \in V_{h} \quad \text { with } \quad \operatorname{supp} v \subset B_{d}
$$

for a ball $B_{d} \subset \Omega$ of radius d (implicitly assumed sufficiently large compared to h). Then

$$
\begin{aligned}
\left\|\nabla\left(u-u_{h}\right)\right\|_{L^{2}\left(B_{d / 4}\right)} & \leq C \inf _{\chi \in V_{h}} \mid\|u-\chi\|_{1, B_{d}}+C d^{-1}\left\|u-u_{h}\right\|_{L^{2}\left(B_{d}\right)} \\
& \leq C \inf _{\chi \in V_{h}}\|\nabla(u-\chi)\|_{L^{2}\left(B_{d}\right)}+C d^{-1}\left\|u-u_{h}\right\|_{L^{2}\left(B_{d}\right)} .
\end{aligned}
$$


Proof The second bound (D.6) follows from (D.5) by an application of the (second) Poincaré inequality.

Let $\omega \in C^{\infty}$ with $\operatorname{supp} \chi \subset B_{d}$ be such that $\omega \equiv 1$ on $B_{d / 2}$ and assume (D.1). Define

$$
\widetilde{u}:=\omega u
$$

and let $\widetilde{u}_{h} \in V_{h} \cap H_{0}^{1}\left(B_{d}\right)$ be its Galerkin approximation on $V_{h} \cap B_{0}^{1}\left(B_{d}\right)$, i.e.,

$$
a\left(\widetilde{u}-\widetilde{u}_{h}, v\right)=0 \quad \forall v \in V_{h} \cap H_{0}^{1}\left(B_{d}\right) .
$$

Then it is classical (and easy to see) that

$$
\int_{B_{d}} \nabla \widetilde{u}_{h} \cdot\left(\mathbf{A} \nabla \widetilde{u}_{h}\right) \leq \int_{B_{d}} \nabla \widetilde{u} \cdot(\mathbf{A} \nabla \widetilde{u})
$$

In particular, we get

$$
\left|\widetilde{u}_{h}\right|_{H^{1}\left(B_{d}\right)} \leq C|\widetilde{u}|_{H^{1}\left(B_{d}\right)} \lesssim|\|u\||_{1, B_{d}}
$$

Next, we write

$$
u-u_{h}=\left(\widetilde{u}-\widetilde{u}_{h}\right)+\left(\widetilde{u}_{h}-u_{h}\right) \quad \text { in } B_{d / 2}
$$

and estimate each of the two terms separately. For the first one, we employ (D.7) to get

$$
\left\|\nabla\left(\widetilde{u}-\widetilde{u}_{h}\right)\right\|_{L^{2}\left(B_{d / 2}\right)} \lesssim\|\nabla \widetilde{u}\|_{L^{2}\left(B_{d}\right)} \lesssim\|u\|_{1, B_{d}} .
$$

For the second term in (D.8), we observe that $\widetilde{u}_{h}-u_{h}$ is discrete harmonic in $B_{d / 2}$ (in fact, almost in $B_{d}$ ) since

$$
a\left(\widetilde{u}_{h}-u_{h}, v\right)=a(\widetilde{u}-u, v)=0 \quad \forall v \in V_{h} \cap H_{0}^{1}\left(B_{d / 2}\right) .
$$

Therefore, Lemma D.2 is applicable and yields in view of $\omega \equiv 1$ on $B_{d / 2}$ :

$$
\begin{aligned}
\left\|\nabla\left(\widetilde{u}_{h}-u_{h}\right)\right\|_{L^{2}\left(B_{d / 4}\right)} & \leq C d^{-1}\left\|\widetilde{u}_{h}-u_{h}\right\|_{L^{2}\left(B_{d / 2}\right)} \\
& \leq C d^{-1}\left\|\widetilde{u}_{h}-u\right\|_{L^{2}\left(B_{d / 2}\right)}+C d^{-1}\left\|u-u_{h}\right\|_{L^{2}\left(B_{d / 2}\right)} \\
& =C d^{-1}\left\|\widetilde{u}_{h}-\widetilde{u}\right\|_{L^{2}\left(B_{d / 2}\right)}+C d^{-1}\left\|u-u_{h}\right\|_{L^{2}\left(B_{d / 2}\right)},
\end{aligned}
$$

where in the last step we exploited $\left.\widetilde{u}\right|_{B_{d / 2}}=\left.u\right|_{B_{d / 2}}$ due to $\left.\omega\right|_{B_{d / 2}} \equiv 1$. Since $\widetilde{u}_{h}$, $\widetilde{u} \in H_{0}^{1}\left(B_{d}\right)$, a Poincaré inequality together with (D.7) produces

$$
\begin{aligned}
\left\|\nabla\left(\widetilde{u}_{h}-u_{h}\right)\right\|_{L^{2}\left(B_{d / 4}\right)} & \lesssim\left\|\nabla\left(\widetilde{u}_{h}-\widetilde{u}\right)\right\|_{L^{2}\left(B_{d}\right)}+d^{-1}\left\|u-u_{h}\right\|_{L^{2}\left(B_{d / 2}\right)} \\
& \lesssim\|u\|_{1, B_{d}}+d^{-1}\left\|u-u_{h}\right\|_{L^{2}\left(B_{d / 2}\right)}
\end{aligned}
$$

Combining (D.9) and (D.10) yields again with $\omega \equiv 1$ on $B_{d / 2}$

$$
\begin{aligned}
\left\|\nabla\left(u-u_{h}\right)\right\|_{L^{2}\left(B_{d / 4}\right)} & \leq\left\|\nabla\left(\widetilde{u}-\widetilde{u}_{h}\right)\right\|_{L^{2}\left(B_{d / 4}\right)}+\left\|\nabla\left(\widetilde{u}_{h}-u_{h}\right)\right\|_{L^{2}\left(B_{d / 4}\right)} \\
& \lesssim\|u\|_{1, B_{d}}+d^{-1}\left\|u-u_{h}\right\|_{L^{2}\left(B_{d}\right)} .
\end{aligned}
$$

The final step consists in noting for arbitrary $\chi \in V_{h}$ that $u-u_{h}=(u-\chi)+\left(\chi-u_{h}\right)$ so that an application of (D.11) applied to $u-\chi$ yields

$$
\left\|\nabla\left(u-u_{h}\right)\right\|_{L^{2}\left(B_{d / 4}\right)} \lesssim\|u-\chi\|_{1, B_{d}}+d^{-1}\left\|u-u_{h}\right\|_{L^{2}\left(B_{d}\right)} .
$$


D.2 The boundary case

We now check to what extent the above results extend up to the boundary. We Let $\Gamma$ be (a part of) the boundary $\partial \Omega$. We denote by

$$
D_{d}=B_{d} \cap \Omega
$$

semiballs of radius $d$, where the implict assumption is always that the center of $D_{d}$ lies on $\Gamma$. Another implicit assumption in the following is that for all semiball appearing in the following, we assume

$$
\partial D_{d} \cap \partial \Omega \subset \Gamma
$$

We define $\|u\|_{1, D_{d}}:=|u|_{H^{1}\left(D_{d}\right)}+\frac{1}{d}\|u\|_{L^{2}\left(D_{d}\right)}$. We employ a space $V_{h}$ which satisfies

$$
V_{h} \subset S^{k, 1}(\mathcal{T}),\left.\quad v\right|_{\Gamma}=0 \quad \forall v \in V_{h} .
$$

We employ the following observation: For a semiball $D_{d} \subset B_{d}$ (same center) as described above, $\omega \in C_{0}^{\infty}\left(B_{d}\right)$, and $u \in V_{h}$, we have $\omega u \in H_{0}^{1}\left(D_{d}\right)$.

Compared to Lemma D.4 the cut-off function $\omega$ may be $\equiv 1$ near parts of $\Gamma$ :

Lemma D.4 (superapproximation on semiballs) Assume (D.12). Let $D_{d} \subset$ $\Omega$ be a semiball of radius $d$. Let $\omega \in C^{\infty}\left(\mathbb{R}^{n}\right)$ with $\operatorname{supp} \omega \subset B_{d / 2}$ and (D.1). Then for every $u \in V_{h}$ the interpolant $I\left(\omega^{2} u\right) \in S^{k, 1}(\mathcal{T}) \cap H_{0}^{1}\left(D_{d}\right)$ satisfies

$$
\begin{aligned}
\left|\omega^{2} u-I\left(\omega^{2} u\right)\right|_{H^{1}\left(D_{d}\right)} & \leq C \frac{h}{d}\|u\|_{1, D_{d}}, \\
\frac{1}{d}\left\|\omega^{2} u-I\left(\omega^{2} u\right)\right\|_{L^{2}\left(D_{d}\right)} & \leq C\left(\frac{h}{d}\right)^{2}\|u\|_{1, D_{d}} .
\end{aligned}
$$

We assume implicitly that $d>h$ is sufficiently large.

Proof Follows by the same arguments as in Lemma D.1

The next result shows an inverse estimate for "discrete harmonic" functions:

Lemma D.5 Assume (D.12). Let $u_{h} \in V_{h}$ satisfy

$$
a\left(u_{h}, v\right)=0 \quad \forall v \in V_{h} \quad \text { with } \quad \operatorname{supp} v \subset \overline{D_{d}}
$$

for a semiball $D_{d} \subset \Omega$ of radius d (implicitly assumed sufficiently large compared to h). Then

$$
\left\|\nabla u_{h}\right\|_{L^{2}\left(D_{d / 2}\right)} \leq C d^{-1}\left\|u_{h}\right\|_{L^{2}\left(D_{d}\right)} .
$$

Proof Again, this follows by tracing the arguments in the proof of Lemma D.5

Theorem D.6 Assume (D.12). Let $u \in H^{1}(\Omega)$ with $\left.u\right|_{\Gamma}=0$ and $u_{h} \in V_{h}$ be such that

$$
a\left(u-u_{h}, v\right)=0 \quad \forall v \in V_{h} \quad \text { with } \quad \operatorname{supp} v \subset \overline{D_{d}}
$$

for a semiball $D_{d} \subset \Omega$ of radius d (implicitly assumed sufficiently large compared to h). Then

$$
\begin{aligned}
\left\|\nabla\left(u-u_{h}\right)\right\|_{L^{2}\left(D_{d / 4}\right)} & \leq C \inf _{\chi \in V_{h}}\|u-\chi\|_{1, D_{d}}+C d^{-1}\left\|u-u_{h}\right\|_{L^{2}\left(D_{d}\right)} \\
& \leq C \inf _{\chi \in V_{h}}\|\nabla(u-\chi)\|_{L^{2}\left(D_{d}\right)}+C d^{-1}\left\|u-u_{h}\right\|_{L^{2}\left(D_{d}\right)} .
\end{aligned}
$$

Proof The second inequality follows again from a Poincaré inequality (the first one, this time). The first inequality follows again from tracing the arguments of the proof of Theorem D.3. 


\section{E Detailed numerics}

E.1 Slit domain

The geometry is a slit domain

$$
\Omega=\Omega^{S}:=(-1,1)^{2} \backslash[0,1) \times\{0\} .
$$

The exact solution is given by

$$
\left|x-x_{0}\right|^{\alpha} \sin (a \pi \phi)
$$

for different choices of the parameters $\alpha$ and $x_{0}$ (and $a$ ).

The inhomogeneous Dirichlet boundary conditions are realized by nodal interpolation. The equation considered is

$$
-\Delta u=f
$$

Starting from a coarse mesh, we perform a sequence of uniform (red) refinements. We consider a lowest order discretization, i.e., $k=1$.

Strictly speaking, the slit domain is not covered by our theory. Also not covered by our theory are the variational crimes associated with approximating the inhomogeneous Dirichlet data. Nevertheless, we expect the convergence behavior detailed in Corollary 4.3 to be an good description of the actual convergence behavior. We assume that the global regularity of the solution $u$ is described by $s=1+\alpha$ (actually, it is $1+\alpha-\varepsilon$ for all $\varepsilon>0$ ). Corollary 4.3 then lets us expect for the two cases $x_{0}=(0,0)$ and $x_{0} \neq(0,0)$ the following convergence rates:

$$
\begin{aligned}
& x_{0}=(0,0) \Longrightarrow \tau=\min \{2,1+\alpha,-1+1 / 2+(1+\alpha)\}=\min \{2,1 / 2+\alpha\} \\
& x_{0} \neq(0,0) \Longrightarrow \tau=\min \{2,1+\alpha,-1+1 / 2+\infty\}=\min \{2,1+\alpha\}
\end{aligned}
$$

In the following Tables E.1 E.7. we vary the parameter $\alpha$. In each table separately we vary the location. The locations under investigation are $\left(x_{0}, y_{0}\right)=(0,0)$, $\left(x_{0}, y_{0}\right)=(0.5,0)$ and $\left(x_{0}, y_{0}\right)=(0,1)$. We observe that the theoretical convergence rates are mostly achieved in our numerical simulations.

\begin{tabular}{|r||c|c||c|c||c|c|}
\hline \multicolumn{1}{|c||}{} & \multicolumn{2}{c||}{$\begin{array}{c}\left(x_{0}, y_{0}\right)=(0,0) \\
a=\pi / 2\end{array}$} & \multicolumn{2}{c||}{$\begin{array}{c}\left(x_{0}, y_{0}\right)=(0.5,0) \\
a=\pi\end{array}$} & \multicolumn{2}{c|}{$\begin{array}{c}\left(x_{0}, y_{0}\right)=(0,1) \\
a=\pi\end{array}$} \\
\hline DOFs & $L^{2}$-error & rate & $L^{2}$-error & rate & $L^{2}$-error & rate \\
\hline 97 & $2.9124 \mathrm{e}-02$ & - & $3.8405 \mathrm{e}-02$ & - & $3.0468 \mathrm{e}-02$ & - \\
348 & $1.5745 \mathrm{e}-02$ & 0.89 & $1.0451 \mathrm{e}-02$ & 1.88 & $1.2883 \mathrm{e}-02$ & 1.24 \\
1.315 & $8.1422 \mathrm{e}-03$ & 0.95 & $4.8926 \mathrm{e}-03$ & 1.10 & $5.2831 \mathrm{e}-03$ & 1.29 \\
5.109 & $4.1322 \mathrm{e}-03$ & 0.98 & $2.1508 \mathrm{e}-03$ & 1.19 & $2.0814 \mathrm{e}-03$ & 1.34 \\
20.137 & $2.0799 \mathrm{e}-03$ & 0.99 & $8.1046 \mathrm{e}-04$ & 1.41 & $7.9896 \mathrm{e}-04$ & 1.38 \\
79.953 & $1.0430 \mathrm{e}-03$ & 1.00 & $3.0969 \mathrm{e}-04$ & 1.39 & $3.0187 \mathrm{e}-04$ & 1.40 \\
318.625 & $5.2221 \mathrm{e}-04$ & 1.00 & $1.1780 \mathrm{e}-04$ & 1.39 & $1.1288 \mathrm{e}-04$ & 1.42 \\
1.272 .129 & $2.6125 \mathrm{e}-04$ & 1.00 & $4.1750 \mathrm{e}-05$ & 1.50 & $4.1903 \mathrm{e}-05$ & 1.43 \\
5.083 .777 & $1.3066 \mathrm{e}-04$ & 1.00 & $1.4985 \mathrm{e}-05$ & 1.48 & $1.5472 \mathrm{e}-05$ & 1.44 \\
\hline
\end{tabular}

Table E.1 Slit domain, $k=1$ : Influence of the position of singularity for $\alpha=0.5$. 


\begin{tabular}{|r||c|c||c|c||c|c|}
\hline \multicolumn{1}{|c||}{} & \multicolumn{2}{c||}{$\begin{array}{c}\left(x_{0}, y_{0}\right)=(0,0) \\
a=\pi / 2\end{array}$} & \multicolumn{2}{c||}{$\begin{array}{c}\left(x_{0}, y_{0}\right)=(0.5,0) \\
a=\pi\end{array}$} & \multicolumn{2}{c|}{$\begin{array}{c}\left(x_{0}, y_{0}\right)=(0,1) \\
a=\pi\end{array}$} \\
\hline DOFs & $L^{2}$-error & rate & $L^{2}$-error & rate & $L^{2}$-error & rate \\
\hline 97 & $1.1642 \mathrm{e}-02$ & - & $1.6577 \mathrm{e}-02$ & - & $1.6031 \mathrm{e}-02$ & - \\
348 & $5.6371 \mathrm{e}-03$ & 1.05 & $6.5153 \mathrm{e}-03$ & 1.35 & $6.0796 \mathrm{e}-03$ & 1.40 \\
1.315 & $2.6059 \mathrm{e}-03$ & 1.11 & $2.3569 \mathrm{e}-03$ & 1.47 & $2.2272 \mathrm{e}-03$ & 1.45 \\
5.109 & $1.1835 \mathrm{e}-03$ & 1.14 & $8.4203 \mathrm{e}-04$ & 1.48 & $7.8285 \mathrm{e}-04$ & 1.51 \\
20.137 & $5.3296 \mathrm{e}-04$ & 1.15 & $2.7392 \mathrm{e}-04$ & 1.62 & $2.6776 \mathrm{e}-04$ & 1.55 \\
79.953 & $2.3888 \mathrm{e}-04$ & 1.16 & $9.1902 \mathrm{e}-05$ & 1.58 & $9.0042 \mathrm{e}-05$ & 1.57 \\
318.625 & $1.0679 \mathrm{e}-04$ & 1.16 & $3.0823 \mathrm{e}-05$ & 1.58 & $2.9942 \mathrm{e}-05$ & 1.59 \\
1.272 .129 & $4.7666 \mathrm{e}-05$ & 1.16 & $9.6773 \mathrm{e}-06$ & 1.67 & $9.8788 \mathrm{e}-06$ & 1.60 \\
5.083 .777 & $2.1257 \mathrm{e}-05$ & 1.16 & $3.1032 \mathrm{e}-06$ & 1.64 & $3.2407 \mathrm{e}-06$ & 1.61 \\
\hline
\end{tabular}

Table E.2 Slit domain, $k=1$ : Influence of the position of singularity for $\alpha=2 / 3$.

\begin{tabular}{|c|c|c|c|c|c|c|}
\hline & \multicolumn{2}{|c|}{$\begin{array}{c}\left(x_{0}, y_{0}\right)=(0,0) \\
a=\pi / 2\end{array}$} & \multicolumn{2}{|c|}{$\begin{aligned}\left(x_{0}, y_{0}\right) & =(0.5,0) \\
a & =\pi\end{aligned}$} & \multicolumn{2}{|c|}{$\begin{array}{c}\left(x_{0}, y_{0}\right)=(0,1) \\
a=\pi\end{array}$} \\
\hline DOFs & $L^{2}$-error & rate & $L^{2}$-error & rate & $L^{2}$-error & rate \\
\hline 97 & $6.1391 \mathrm{e}-03$ & - & $1.1088 \mathrm{e}-02$ & - & $1.0692 \mathrm{e}-02$ & - \\
\hline 348 & $2.8187 \mathrm{e}-03$ & 1.12 & $4.1329 \mathrm{e}-03$ & 1.42 & $3.8553 \mathrm{e}-03$ & 1.47 \\
\hline 1.315 & $1.2351 \mathrm{e}-03$ & 1.19 & $1.4164 \mathrm{e}-03$ & 1.54 & $1.3388 \mathrm{e}-03$ & 1.53 \\
\hline 5.109 & $5.3338 \mathrm{e}-04$ & 1.21 & $4.7830 \mathrm{e}-04$ & 1.57 & $4.4562 \mathrm{e}-04$ & 1.59 \\
\hline 20.137 & $2.2846 \mathrm{e}-04$ & 1.22 & $1.4725 \mathrm{e}-04$ & 1.70 & $1.4420 \mathrm{e}-04$ & 1.63 \\
\hline 79.953 & $9.7267 \mathrm{e}-05$ & 1.23 & $4.6683 \mathrm{e}-05$ & 1.66 & $4.5843 \mathrm{e}-05$ & 1.65 \\
\hline 318.625 & $4.1233 \mathrm{e}-05$ & 1.24 & $1.4761 \mathrm{e}-05$ & 1.66 & $1.4401 \mathrm{e}-05$ & 1.67 \\
\hline 1.272 .129 & $1.7428 \mathrm{e}-05$ & 1.24 & $4.3773 \mathrm{e}-06$ & 1.75 & $4.4861 \mathrm{e}-06$ & 1.68 \\
\hline 5.083 .777 & $7.3524 \mathrm{e}-06$ & 1.25 & $1.3285 \mathrm{e}-06$ & 1.72 & $1.3889 \mathrm{e}-06$ & 1.69 \\
\hline
\end{tabular}

Table E.3 Slit domain, $k=1$ : Influence of the position of singularity for $\alpha=0.75$.

\begin{tabular}{|r||c|c||c|c||c|c|}
\hline \multicolumn{1}{|c||}{} & \multicolumn{2}{c||}{$\begin{array}{c}\left(x_{0}, y_{0}\right)=(0,0) \\
a=\pi / 2\end{array}$} & \multicolumn{2}{c||}{$\begin{array}{c}\left(x_{0}, y_{0}\right)=(0.5,0) \\
a=\pi\end{array}$} & \multicolumn{2}{c|}{$\begin{array}{c}\left(x_{0}, y_{0}\right)=(0,1) \\
a=\pi\end{array}$} \\
\hline DOFs & $L^{2}$-error & rate & $L^{2}$-error & rate & $L^{2}$-error & rate \\
\hline 97 & $4.1949 \mathrm{e}-03$ & - & $3.0111 \mathrm{e}-04$ & - & $2.9784 \mathrm{e}-04$ & - \\
348 & $1.4605 \mathrm{e}-03$ & 1.52 & $9.9257 \mathrm{e}-05$ & 1.60 & $9.3618 \mathrm{e}-05$ & 1.67 \\
1.315 & $4.8756 \mathrm{e}-04$ & 1.58 & $2.9679 \mathrm{e}-05$ & 1.74 & $2.8033 \mathrm{e}-05$ & 1.74 \\
5.109 & $1.5909 \mathrm{e}-04$ & 1.62 & $8.6201 \mathrm{e}-06$ & 1.78 & $8.0205 \mathrm{e}-06$ & 1.81 \\
20.137 & $5.1667 \mathrm{e}-05$ & 1.62 & $2.2994 \mathrm{e}-06$ & 1.91 & $2.2266 \mathrm{e}-06$ & 1.85 \\
79.953 & $1.6874 \mathrm{e}-05$ & 1.61 & $6.2533 \mathrm{e}-07$ & 1.88 & $6.0606 \mathrm{e}-07$ & 1.88 \\
318.625 & $5.5687 \mathrm{e}-06$ & 1.60 & $1.6832 \mathrm{e}-07$ & 1.89 & $1.6270 \mathrm{e}-07$ & 1.90 \\
1.272 .129 & $1.8596 \mathrm{e}-06$ & 1.58 & $4.3035 \mathrm{e}-08$ & 1.97 & $4.3240 \mathrm{e}-08$ & 1.91 \\
5.083 .777 & $6.2798 \mathrm{e}-07$ & 1.57 & $1.1235 \mathrm{e}-08$ & 1.94 & $1.1403 \mathrm{e}-08$ & 1.92 \\
\hline
\end{tabular}

Table E.4 Slit domain, $k=1$ : Influence of the position of singularity for $\alpha=1.01$. 


\begin{tabular}{|r||c|c||c|c||c|c|}
\hline \multicolumn{1}{|c||}{} & \multicolumn{2}{c||}{$\begin{array}{c}\left(x_{0}, y_{0}\right)=(0,0) \\
a=\pi / 2\end{array}$} & \multicolumn{2}{c||}{$\begin{array}{c}\left(x_{0}, y_{0}\right)=(0.5,0) \\
a=\pi\end{array}$} & \multicolumn{2}{c|}{$\begin{array}{c}\left(x_{0}, y_{0}\right)=(0,1) \\
a=\pi\end{array}$} \\
\hline DOFs & $L^{2}$-error & rate & $L^{2}$-error & rate & $L^{2}$-error & rate \\
\hline 97 & $5.7534 \mathrm{e}-03$ & - & $2.8888 \mathrm{e}-03$ & - & $2.8799 \mathrm{e}-03$ & - \\
348 & $1.9412 \mathrm{e}-03$ & 1.57 & $9.1606 \mathrm{e}-04$ & 1.66 & $8.6618 \mathrm{e}-04$ & 1.73 \\
1.315 & $6.2583 \mathrm{e}-04$ & 1.63 & $2.6267 \mathrm{e}-04$ & 1.80 & $2.4731 \mathrm{e}-04$ & 1.81 \\
5.109 & $1.9689 \mathrm{e}-04$ & 1.67 & $7.2833 \mathrm{e}-05$ & 1.85 & $6.7463 \mathrm{e}-05$ & 1.87 \\
20.137 & $6.1446 \mathrm{e}-05$ & 1.68 & $1.8673 \mathrm{e}-05$ & 1.96 & $1.7871 \mathrm{e}-05$ & 1.92 \\
79.953 & $1.9191 \mathrm{e}-05$ & 1.68 & $4.8621 \mathrm{e}-06$ & 1.94 & $4.6455 \mathrm{e}-06$ & 1.94 \\
318.625 & $6.0229 \mathrm{e}-06$ & 1.67 & $1.2518 \mathrm{e}-06$ & 1.96 & $1.1921 \mathrm{e}-06$ & 1.96 \\
1.272 .129 & $1.9023 \mathrm{e}-06$ & 1.66 & $3.0913 \mathrm{e}-07$ & 2.02 & $3.0311 \mathrm{e}-07$ & 1.98 \\
5.083 .777 & $6.0474 \mathrm{e}-07$ & 1.65 & $7.7720 \mathrm{e}-08$ & 1.99 & $7.6552 \mathrm{e}-08$ & 1.99 \\
\hline
\end{tabular}

Table E.5 Slit domain, $k=1$ : Influence of the position of singularity for $\alpha=10 / 9$.

\begin{tabular}{|r||c|c||c|c||c|c|}
\hline \multicolumn{1}{|c||}{} & \multicolumn{2}{c||}{$\begin{array}{c}\left(x_{0}, y_{0}\right)=(0,0) \\
a=\pi / 2\end{array}$} & \multicolumn{2}{c||}{$\begin{array}{c}\left(x_{0}, y_{0}\right)=(0.5,0) \\
a=\pi\end{array}$} & \multicolumn{2}{c|}{$\begin{array}{c}\left(x_{0}, y_{0}\right)=(0,1) \\
\end{array}$} \\
\hline DOFs & $L^{2}$-error & rate & $L^{2}$-error & rate & $L^{2}$-error & rate \\
\hline 97 & $7.3549 \mathrm{e}-03$ & - & $6.3401 \mathrm{e}-03$ & - & $6.3849 \mathrm{e}-03$ & - \\
348 & $2.2414 \mathrm{e}-03$ & 1.71 & $1.8792 \mathrm{e}-03$ & 1.75 & $1.7790 \mathrm{e}-03$ & 1.84 \\
1.315 & $6.4849 \mathrm{e}-04$ & 1.79 & $5.0365 \mathrm{e}-04$ & 1.90 & $4.6905 \mathrm{e}-04$ & 1.92 \\
5.109 & $1.8251 \mathrm{e}-04$ & 1.83 & $1.3007 \mathrm{e}-04$ & 1.95 & $1.1878 \mathrm{e}-04$ & 1.98 \\
20.137 & $5.0718 \mathrm{e}-05$ & 1.85 & $3.1798 \mathrm{e}-05$ & 2.03 & $2.9443 \mathrm{e}-05$ & 2.01 \\
79.953 & $1.4021 \mathrm{e}-05$ & 1.85 & $7.8665 \mathrm{e}-06$ & 2.02 & $7.2227 \mathrm{e}-06$ & 2.03 \\
318.625 & $3.8699 \mathrm{e}-06$ & 1.86 & $1.9356 \mathrm{e}-06$ & 2.02 & $1.7642 \mathrm{e}-06$ & 2.03 \\
1.272 .129 & $1.0682 \mathrm{e}-06$ & 1.86 & $4.6924 \mathrm{e}-07$ & 2.04 & $4.3055 \mathrm{e}-07$ & 2.03 \\
5.083 .777 & $2.9514 \mathrm{e}-07$ & 1.86 & $1.1524 \mathrm{e}-07$ & 2.03 & $1.0519 \mathrm{e}-07$ & 2.03 \\
\hline
\end{tabular}

Table E.6 Slit domain, $k=1$ : Influence of the position of singularity for $\alpha=4 / 3$.

\begin{tabular}{|r||c|c||c|c||c|c|}
\hline \multicolumn{1}{|c||}{} & \multicolumn{2}{c||}{$\begin{array}{c}\left(x_{0}, y_{0}\right)=(0,0) \\
a=\pi / 2\end{array}$} & \multicolumn{2}{c||}{$\begin{array}{c}\left(x_{0}, y_{0}\right)=(0.5,0) \\
a=\pi\end{array}$} & \multicolumn{2}{c|}{$\begin{array}{c}\left(x_{0}, y_{0}\right)=(0,1) \\
a=\pi\end{array}$} \\
\hline DOFs & $L^{2}$-error & rate & $L^{2}$-error & rate & $L^{2}$-error & rate \\
\hline 97 & $7.5901 \mathrm{e}-03$ & - & $7.6006 \mathrm{e}-03$ & - & $7.6553 \mathrm{e}-03$ & - \\
348 & $2.1664 \mathrm{e}-03$ & 1.81 & $2.1751 \mathrm{e}-03$ & 1.81 & $2.0530 \mathrm{e}-03$ & 1.90 \\
1.315 & $5.8638 \mathrm{e}-04$ & 1.89 & $5.6614 \mathrm{e}-04$ & 1.94 & $5.2238 \mathrm{e}-04$ & 1.97 \\
5.109 & $1.5450 \mathrm{e}-04$ & 1.92 & $1.4246 \mathrm{e}-04$ & 1.99 & $1.2877 \mathrm{e}-04$ & 2.02 \\
20.137 & $4.0197 \mathrm{e}-05$ & 1.94 & $3.4615 \mathrm{e}-05$ & 2.04 & $3.1388 \mathrm{e}-05$ & 2.04 \\
79.953 & $1.0396 \mathrm{e}-05$ & 1.95 & $8.5086 \mathrm{e}-06$ & 2.02 & $7.6403 \mathrm{e}-06$ & 2.04 \\
318.625 & $2.6803 \mathrm{e}-06$ & 1.96 & $2.0921 \mathrm{e}-06$ & 2.02 & $1.8651 \mathrm{e}-06$ & 2.03 \\
1.272 .129 & $6.8978 \mathrm{e}-07$ & 1.96 & $5.1307 \mathrm{e}-07$ & 2.03 & $4.5726 \mathrm{e}-07$ & 2.03 \\
5.083 .777 & $1.7730 \mathrm{e}-07$ & 1.96 & $1.2691 \mathrm{e}-07$ & 2.02 & $1.1258 \mathrm{e}-07$ & 2.02 \\
\hline
\end{tabular}

Table E.7 Slit domain, $k=1$ : Influence of the position of singularity for $\alpha=1.5$. 
E.2 L-shaped domain

The geometry is an L-shaped domain:

$$
\Omega=\Omega^{L}:=(-1,1)^{2} \backslash[0,1) \times(-1,0] .
$$

The exact solution is given by

$$
\left|x-x_{0}\right|^{\alpha} \sin (a \pi \phi)
$$

for different choices of the parameters $\alpha, x_{0}$, and $a$.

\section{E.2.1 Lowest order discretization $k=1$}

Structurally, the situation is similar to the situation in Section E.1. From Corollary 4.3 we expect the following convergence rates:

$$
\begin{aligned}
& x_{0}=(0,0) \Longrightarrow \tau=\min \{2,1+\alpha,-1+2 / 3+(1+\alpha)\}=\min \{2,2 / 3+\alpha\} \\
& x_{0} \neq(0,0) \Longrightarrow \tau=\min \{2,1+\alpha,-1+2 / 3+\infty\}=\min \{2,1+\alpha\}
\end{aligned}
$$

\begin{tabular}{|c|c|c|c|c|c|c|}
\hline & \multicolumn{2}{|c|}{$\begin{array}{c}\left(x_{0}, y_{0}\right)=(0,0) \\
a=\pi / 2\end{array}$} & \multicolumn{2}{|c|}{$\begin{array}{c}\left(x_{0}, y_{0}\right)=(0.5,0) \\
a=\pi\end{array}$} & \multicolumn{2}{|c|}{$\begin{array}{c}\left(x_{0}, y_{0}\right)=(0,1) \\
a=\pi\end{array}$} \\
\hline DOFs & $L^{2}$-error & rate & $L^{2}$-error & rate & $L^{2}$-error & rate \\
\hline 81 & $1.1719 \mathrm{e}-02$ & - & $1.0132 \mathrm{e}-02$ & - & $9.5383 \mathrm{e}-03$ & - \\
\hline 289 & $5.4059 \mathrm{e}-03$ & 1.12 & $4.0619 \mathrm{e}-03$ & 1.32 & $3.4014 \mathrm{e}-03$ & 1.49 \\
\hline 1.089 & $2.3165 \mathrm{e}-03$ & 1.22 & $1.4839 \mathrm{e}-03$ & 1.45 & $1.1426 \mathrm{e}-03$ & 1.57 \\
\hline 4.225 & $9.5790 \mathrm{e}-04$ & 1.27 & $5.1844 \mathrm{e}-04$ & 1.52 & $3.7569 \mathrm{e}-04$ & 1.60 \\
\hline 16.641 & $3.8922 \mathrm{e}-04$ & 1.30 & $1.7681 \mathrm{e}-04$ & 1.55 & $1.2222 \mathrm{e}-04$ & 1.62 \\
\hline 66.049 & $1.5663 \mathrm{e}-04$ & 1.31 & $5.9408 \mathrm{e}-05$ & 1.57 & $3.9513 \mathrm{e}-05$ & 1.63 \\
\hline 263.169 & $6.2682 \mathrm{e}-05$ & 1.32 & $1.9762 \mathrm{e}-05$ & 1.59 & $1.2720 \mathrm{e}-05$ & 1.64 \\
\hline 1.050 .625 & $2.5002 \mathrm{e}-05$ & 1.33 & $6.5263 \mathrm{e}-06$ & 1.60 & $4.0824 \mathrm{e}-06$ & 1.64 \\
\hline 4.198 .401 & $9.9525 \mathrm{e}-06$ & 1.33 & $2.1437 \mathrm{e}-06$ & 1.61 & $1.3072 \mathrm{e}-06$ & 1.64 \\
\hline
\end{tabular}

Also in this case the numerical rates depicted in Tables E.8 E.13 are very close to the rates expected by our theory.

Table E.8 L-domain, $k=1$ : Influence of the position of singularity for $\alpha=2 / 3$. 


\begin{tabular}{|r||c|c||c|c||c|c|}
\hline \multicolumn{1}{|c||}{} & \multicolumn{2}{c||}{\begin{tabular}{c}
$\left(x_{0}, y_{0}\right)=(0,0)$ \\
\multicolumn{1}{|c||}{$a=\pi / 2$}
\end{tabular}} & \multicolumn{2}{c||}{$\begin{array}{c}\left(x_{0}, y_{0}\right)=(0.5,0) \\
a=\pi\end{array}$} & \multicolumn{2}{c|}{$\begin{array}{c}\left(x_{0}, y_{0}\right)=(0,1) \\
a=\pi\end{array}$} \\
\hline DOFs & $L^{2}$-error & rate & $L^{2}$-error & rate & $L^{2}$-error & rate \\
\hline 81 & $6.1585 \mathrm{e}-03$ & - & $6.8141 \mathrm{e}-03$ & - & $6.2506 \mathrm{e}-03$ & - \\
289 & $2.6986 \mathrm{e}-03$ & 1.19 & $2.5648 \mathrm{e}-03$ & 1.41 & $2.1211 \mathrm{e}-03$ & 1.56 \\
1.089 & $1.1123 \mathrm{e}-03$ & 1.28 & $8.8428 \mathrm{e}-04$ & 1.54 & $6.7413 \mathrm{e}-04$ & 1.65 \\
4.225 & $4.4037 \mathrm{e}-04$ & 1.34 & $2.9202 \mathrm{e}-04$ & 1.60 & $2.0903 \mathrm{e}-04$ & 1.69 \\
16.641 & $1.7107 \mathrm{e}-04$ & 1.36 & $9.4164 \mathrm{e}-05$ & 1.63 & $6.4027 \mathrm{e}-05$ & 1.71 \\
66.049 & $6.5689 \mathrm{e}-05$ & 1.38 & $2.9909 \mathrm{e}-05$ & 1.65 & $1.9471 \mathrm{e}-05$ & 1.72 \\
263.169 & $2.5030 \mathrm{e}-05$ & 1.39 & $9.4012 \mathrm{e}-06$ & 1.67 & $5.8930 \mathrm{e}-06$ & 1.72 \\
1.050 .625 & $9.4877 \mathrm{e}-06$ & 1.40 & $2.9328 \mathrm{e}-06$ & 1.68 & $1.7774 \mathrm{e}-06$ & 1.73 \\
4.198 .401 & $3.5834 \mathrm{e}-06$ & 1.40 & $9.0968 \mathrm{e}-07$ & 1.69 & $5.3475 \mathrm{e}-07$ & 1.73 \\
\hline
\end{tabular}

Table E.9 L-domain, $k=1$ : Influence of the position of singularity for $\alpha=0.75$.

\begin{tabular}{|r||c|c||c|c||c|c|}
\hline \multicolumn{1}{|c||}{} & \multicolumn{2}{c||}{$\begin{array}{c}\left(x_{0}, y_{0}\right)=(0,0) \\
a=\pi / 2\end{array}$} & \multicolumn{2}{c||}{$\begin{array}{c}\left(x_{0}, y_{0}\right)=(0.5,0) \\
a=\pi\end{array}$} & \multicolumn{2}{c|}{$\begin{array}{c}\left(x_{0}, y_{0}\right)=(0,1) \\
\end{array}$} \\
\hline DOFs & $L^{2}$-error & rate & $L^{2}$-error & rate & $L^{2}$-error & rate \\
\hline 81 & $4.6216 \mathrm{e}-03$ & - & $1.8387 \mathrm{e}-04$ & - & $1.6841 \mathrm{e}-04$ & - \\
289 & $1.6860 \mathrm{e}-03$ & 1.45 & $6.0370 \mathrm{e}-05$ & 1.61 & $5.0364 \mathrm{e}-05$ & 1.74 \\
1.089 & $5.4867 \mathrm{e}-04$ & 1.62 & $1.8034 \mathrm{e}-05$ & 1.74 & $1.3883 \mathrm{e}-05$ & 1.86 \\
4.225 & $1.7284 \mathrm{e}-04$ & 1.67 & $5.1378 \mathrm{e}-06$ & 1.81 & $3.6942 \mathrm{e}-06$ & 1.91 \\
16.641 & $5.2963 \mathrm{e}-05$ & 1.71 & $1.4253 \mathrm{e}-06$ & 1.85 & $9.6399 \mathrm{e}-07$ & 1.94 \\
66.049 & $1.5970 \mathrm{e}-05$ & 1.73 & $3.8870 \mathrm{e}-07$ & 1.87 & $2.4842 \mathrm{e}-07$ & 1.96 \\
263.169 & $4.7758 \mathrm{e}-06$ & 1.74 & $1.0474 \mathrm{e}-07$ & 1.89 & $6.3437 \mathrm{e}-08$ & 1.97 \\
1.050 .625 & $1.4238 \mathrm{e}-06$ & 1.75 & $2.7971 \mathrm{e}-08$ & 1.90 & $1.6086 \mathrm{e}-08$ & 1.98 \\
4.198 .401 & $4.2471 \mathrm{e}-07$ & 1.75 & $7.4181 \mathrm{e}-09$ & 1.91 & $4.0561 \mathrm{e}-09$ & 1.99 \\
\hline
\end{tabular}

Table E.10 L-domain, $k=1$ : Influence of the position of singularity for $\alpha=1.01$.

\begin{tabular}{|r||c|c||c|c||c|c|}
\hline \multicolumn{1}{|c||}{} & \multicolumn{2}{c||}{$\left(x_{0}, y_{0}\right)=(0,0)$} & \multicolumn{2}{c||}{$\left(x_{0}, y_{0}\right)=(0.5,0)$} & \multicolumn{2}{c|}{$\left(x_{0}, y_{0}\right)=(0,1)$} \\
& $a=\pi / 2$ & \multicolumn{2}{c||}{$a=\pi$} & \multicolumn{2}{c|}{$a=\pi$} \\
\hline DOFs & $L^{2}$-error & rate & $L^{2}$-error & rate & $L^{2}$-error & rate \\
\hline 81 & $6.5660 \mathrm{e}-03$ & - & $1.7641 \mathrm{e}-03$ & - & $1.6229 \mathrm{e}-03$ & - \\
289 & $2.3309 \mathrm{e}-03$ & 1.49 & $5.5465 \mathrm{e}-04$ & 1.67 & $4.6837 \mathrm{e}-04$ & 1.79 \\
1.089 & $7.3413 \mathrm{e}-04$ & 1.67 & $1.5847 \mathrm{e}-04$ & 1.81 & $1.2424 \mathrm{e}-04$ & 1.91 \\
4.225 & $2.2257 \mathrm{e}-04$ & 1.72 & $4.3172 \mathrm{e}-05$ & 1.88 & $3.1761 \mathrm{e}-05$ & 1.97 \\
16.641 & $6.5650 \mathrm{e}-05$ & 1.76 & $1.1458 \mathrm{e}-05$ & 1.91 & $7.9588 \mathrm{e}-06$ & 2.00 \\
66.049 & $1.9056 \mathrm{e}-05$ & 1.78 & $2.9916 \mathrm{e}-06$ & 1.94 & $1.9695 \mathrm{e}-06$ & 2.01 \\
263.169 & $5.4810 \mathrm{e}-06$ & 1.80 & $7.7249 \mathrm{e}-07$ & 1.95 & $4.8311 \mathrm{e}-07$ & 2.03 \\
1.050 .625 & $1.5690 \mathrm{e}-06$ & 1.80 & $1.9789 \mathrm{e}-07$ & 1.96 & $1.1771 \mathrm{e}-07$ & 2.04 \\
4.198 .401 & $4.4822 \mathrm{e}-07$ & 1.81 & $5.0393 \mathrm{e}-08$ & 1.97 & $2.8532 \mathrm{e}-08$ & 2.04 \\
\hline
\end{tabular}

Table E.11 L-domain, $k=1$ : Influence of the position of singularity for $\alpha=10 / 9$. 


\begin{tabular}{|r||c|c||c|c||c|c|}
\hline \multicolumn{1}{|c||}{} & \multicolumn{2}{c||}{$\begin{array}{c}\left(x_{0}, y_{0}\right)=(0,0) \\
a=\pi / 2\end{array}$} & \multicolumn{2}{c||}{$\begin{array}{c}\left(x_{0}, y_{0}\right)=(0.5,0) \\
a=\pi\end{array}$} & \multicolumn{2}{c|}{$\begin{array}{c}\left(x_{0}, y_{0}\right)=(0,1) \\
a=\pi\end{array}$} \\
\hline DOFs & $L^{2}$-error & rate & $L^{2}$-error & rate & $L^{2}$-error & rate \\
\hline 81 & $8.6776 \mathrm{e}-03$ & - & $3.8962 \mathrm{e}-03$ & - & $3.6446 \mathrm{e}-03$ & - \\
289 & $2.8523 \mathrm{e}-03$ & 1.61 & $1.1374 \mathrm{e}-03$ & 1.78 & $1.0008 \mathrm{e}-03$ & 1.86 \\
1.089 & $8.2870 \mathrm{e}-04$ & 1.78 & $3.0272 \mathrm{e}-04$ & 1.91 & $2.5331 \mathrm{e}-04$ & 1.98 \\
4.225 & $2.3073 \mathrm{e}-04$ & 1.84 & $7.7239 \mathrm{e}-05$ & 1.97 & $6.2153 \mathrm{e}-05$ & 2.03 \\
16.641 & $6.2539 \mathrm{e}-05$ & 1.88 & $1.9331 \mathrm{e}-05$ & 2.00 & $1.5073 \mathrm{e}-05$ & 2.04 \\
66.049 & $1.6688 \mathrm{e}-05$ & 1.91 & $4.7956 \mathrm{e}-06$ & 2.01 & $3.6440 \mathrm{e}-06$ & 2.05 \\
263.169 & $4.4099 \mathrm{e}-06$ & 1.92 & $1.1852 \mathrm{e}-06$ & 2.02 & $8.8167 \mathrm{e}-07$ & 2.05 \\
1.050 .625 & $1.1580 \mathrm{e}-06$ & 1.93 & $2.9260 \mathrm{e}-07$ & 2.02 & $2.1389 \mathrm{e}-07$ & 2.04 \\
4.198 .401 & $3.0279 \mathrm{e}-07$ & 1.94 & $7.2263 \mathrm{e}-08$ & 2.02 & $5.2069 \mathrm{e}-08$ & 2.04 \\
\hline
\end{tabular}

Table E.12 L-domain, $k=1$ : Influence of the position of singularity for $\alpha=4 / 3$.

\begin{tabular}{|r||c|c||c|c||c|c|}
\hline \multicolumn{1}{|c||}{} & \multicolumn{2}{c||}{$\left(x_{0}, y_{0}\right)=(0,0)$} & \multicolumn{2}{c||}{$\left(x_{0}, y_{0}\right)=(0.5,0)$} & \multicolumn{2}{c|}{$\begin{array}{c}\left(x_{0}, y_{0}\right)=(0,1) \\
a=\pi / 2\end{array}$} \\
\hline DOFs & $L^{2}$-error & rate & $L^{2}$-error & rate & $L^{2}$-error & rate \\
\hline 81 & $8.9932 \mathrm{e}-03$ & - & $4.7178 \mathrm{e}-03$ & - & $4.4942 \mathrm{e}-03$ & - \\
289 & $2.8151 \mathrm{e}-03$ & 1.68 & $1.3287 \mathrm{e}-03$ & 1.83 & $1.2166 \mathrm{e}-03$ & 1.89 \\
1.089 & $7.8034 \mathrm{e}-04$ & 1.85 & $3.4367 \mathrm{e}-04$ & 1.95 & $3.0580 \mathrm{e}-04$ & 1.99 \\
4.225 & $2.0751 \mathrm{e}-04$ & 1.91 & $8.5903 \mathrm{e}-05$ & 2.00 & $7.5035 \mathrm{e}-05$ & 2.03 \\
16.641 & $5.3910 \mathrm{e}-05$ & 1.94 & $2.1227 \mathrm{e}-05$ & 2.02 & $1.8321 \mathrm{e}-05$ & 2.03 \\
66.049 & $1.3835 \mathrm{e}-05$ & 1.96 & $5.2331 \mathrm{e}-06$ & 2.02 & $4.4827 \mathrm{e}-06$ & 2.03 \\
263.169 & $3.5256 \mathrm{e}-06$ & 1.97 & $1.2917 \mathrm{e}-06$ & 2.02 & $1.1011 \mathrm{e}-06$ & 2.03 \\
1.050 .625 & $8.9467 \mathrm{e}-07$ & 1.98 & $3.1955 \mathrm{e}-07$ & 2.02 & $2.7158 \mathrm{e}-07$ & 2.02 \\
4.198 .401 & $2.2641 \mathrm{e}-07$ & 1.98 & $7.9238 \mathrm{e}-08$ & 2.01 & $6.7212 \mathrm{e}-08$ & 2.01 \\
\hline
\end{tabular}

Table E.13 L-domain, $k=1$ : Influence of the position of singularity for $\alpha=1.5$. 
E.2.2 Second order discretization $k=2$

All calculations are performed for $x_{0}=(0,0)$ and $a=2 / 3$. Only the singularity parameter $\alpha$ is varied.

Here, we expect the convergence rate

$$
\tau=\min \{3,-1+2 / 3+(1+\alpha)\}=\min \{3,2 / 3+\alpha\} .
$$

Table E.14 shows the numerical results for the second order case in which the received rates are close to the theoretical expected once.

\begin{tabular}{|r||c|c||c|c||c|c||c|c|}
\hline \multicolumn{1}{|c||}{} & \multicolumn{2}{c||}{$\alpha=2 / 3$} & \multicolumn{2}{c||}{$\alpha=3 / 4$} & \multicolumn{2}{c|}{$\alpha=1.01$} & \multicolumn{2}{c|}{$\alpha=10 / 9$} \\
\hline DOFs & $L^{2}$-error & rate & $L^{2}$-error & rate & $L^{2}$-error & rate & $L^{2}$-error & rate \\
\hline 289 & $3.1686 \mathrm{e}-03$ & - & $1.6898 \mathrm{e}-03$ & - & $4.8115 \mathrm{e}-04$ & - & $5.9011 \mathrm{e}-04$ & - \\
1.089 & $1.2099 \mathrm{e}-03$ & 1.39 & $6.0844 \mathrm{e}-04$ & 1.47 & $1.4003 \mathrm{e}-04$ & 1.78 & $1.5596 \mathrm{e}-04$ & 1.92 \\
4.225 & $4.6505 \mathrm{e}-04$ & 1.38 & $2.1881 \mathrm{e}-04$ & 1.48 & $3.7277 \mathrm{e}-05$ & 1.91 & $3.8312 \mathrm{e}-05$ & 2.03 \\
16.641 & $1.8057 \mathrm{e}-04$ & 1.36 & $8.0073 \mathrm{e}-05$ & 1.45 & $9.9546 \mathrm{e}-06$ & 1.90 & $9.3965 \mathrm{e}-06$ & 2.03 \\
66.049 & $7.0635 \mathrm{e}-05$ & 1.35 & $2.9545 \mathrm{e}-05$ & 1.44 & $2.6951 \mathrm{e}-06$ & 1.89 & $2.3314 \mathrm{e}-06$ & 2.01 \\
263.169 & $2.7771 \mathrm{e}-05$ & 1.35 & $1.0960 \mathrm{e}-05$ & 1.43 & $7.4481 \mathrm{e}-07$ & 1.86 & $5.8950 \mathrm{e}-07$ & 1.98 \\
1.050 .625 & $1.0955 \mathrm{e}-05$ & 1.34 & $4.0799 \mathrm{e}-06$ & 1.43 & $2.1075 \mathrm{e}-07$ & 1.82 & $1.5257 \mathrm{e}-07$ & 1.95 \\
\hline
\end{tabular}

\begin{tabular}{|r||c|c||c|c||c|c||c|c|}
\hline \multicolumn{1}{|c||}{} & \multicolumn{2}{c||}{$\alpha=4 / 3$} & \multicolumn{2}{c||}{$\alpha=3 / 2$} & \multicolumn{2}{c||}{$\alpha=2.175$} & \multicolumn{2}{c|}{$\alpha=2.275$} \\
\hline DOFs & $L^{2}$-error & rate & $L^{2}$-error & rate & $L^{2}$-error & rate & $L^{2}$-error & rate \\
\hline 289 & $6.1433 \mathrm{e}-04$ & - & $5.5363 \mathrm{e}-04$ & - & $2.7565 \mathrm{e}-04$ & - & $2.4570 \mathrm{e}-04$ & - \\
1.089 & $1.5136 \mathrm{e}-04$ & 2.02 & $1.3540 \mathrm{e}-04$ & 2.03 & $5.1121 \mathrm{e}-05$ & 2.43 & $4.1696 \mathrm{e}-05$ & 2.56 \\
4.225 & $3.3604 \mathrm{e}-05$ & 2.17 & $2.8521 \mathrm{e}-05$ & 2.25 & $7.5320 \mathrm{e}-06$ & 2.76 & $5.7319 \mathrm{e}-06$ & 2.86 \\
16.641 & $7.7002 \mathrm{e}-06$ & 2.13 & $6.2123 \mathrm{e}-06$ & 2.20 & $1.1051 \mathrm{e}-06$ & 2.77 & $7.8407 \mathrm{e}-07$ & 2.87 \\
66.049 & $1.8014 \mathrm{e}-06$ & 2.10 & $1.3642 \mathrm{e}-06$ & 2.19 & $1.5938 \mathrm{e}-07$ & 2.79 & $1.0553 \mathrm{e}-07$ & 2.89 \\
263.169 & $4.2916 \mathrm{e}-07$ & 2.07 & $3.0106 \mathrm{e}-07$ & 2.18 & $2.2723 \mathrm{e}-08$ & 2.81 & $1.4044 \mathrm{e}-08$ & 2.91 \\
1.050 .625 & $1.0374 \mathrm{e}-07$ & 2.05 & $6.6649 \mathrm{e}-08$ & 2.18 & $3.2138 \mathrm{e}-09$ & 2.82 & $1.8538 \mathrm{e}-09$ & 2.92 \\
\hline
\end{tabular}

\begin{tabular}{|r||c|c|}
\hline \multicolumn{1}{|c||}{} & \multicolumn{2}{c|}{$\alpha=2.375$} \\
\hline DOFs & $L^{2}$-error & rate \\
\hline 289 & $2.2177 \mathrm{e}-04$ & - \\
1.089 & $3.3912 \mathrm{e}-05$ & 2.71 \\
4.225 & $4.3221 \mathrm{e}-06$ & 2.97 \\
16.641 & $5.4888 \mathrm{e}-07$ & 2.98 \\
66.049 & $6.8762 \mathrm{e}-08$ & 3.00 \\
263.169 & $8.5292 \mathrm{e}-09$ & 3.01 \\
1.050 .625 & $1.0497 \mathrm{e}-09$ & 3.02 \\
\hline
\end{tabular}

Table E.14 L-shaped domain, $k=2$ : Influence of $\alpha$ for $a=2 / 3 \pi$ and $\left(x_{0}, y_{0}\right)=(0,0)$. 
E.3 Fichera corner

\section{E.3.1 Smooth solution}

The geometry is

$$
\Omega=\Omega^{F}:=(-1,1)^{3} \backslash[0,1]^{3} .
$$

The discretization is done on lowest order hexahedral elements, regularly refined. The exact solution is prescribed to be the smooth solution

$$
u(x, y, z)=\sin ((x+y) \pi) \cos (2 \pi z) .
$$

\begin{tabular}{|r|c|c|}
\hline DOFs & L2 error & rate \\
\hline 316 & 0.075444 & - \\
3.032 & 0.017182 & 1.96 \\
26.416 & 0.0039376 & 2.04 \\
220.256 & 0.00094597 & 2.02 \\
1.798 .336 & 0.00023208 & 2.01 \\
14.532 .992 & $5.7491 \mathrm{e}-05$ & 2.00 \\
\hline
\end{tabular}

Table E.15 Fichera cube.

\section{E.3.2 Solution of point singularity type}

In the next calculations, the exact solution is given by

$$
u=r^{\alpha},
$$

where $r=\operatorname{dist}\left(x, x_{0}\right)$ measures the distance from the point $x_{0}$, which is varied. The $L^{2}$-error is computed with a tensor product Gauss rule $(5$ points in each coordinate direction).

\begin{tabular}{|r||c|c||c|c|c|c|}
\hline \multicolumn{1}{|c||}{} & \multicolumn{1}{|c||}{$x_{0}=(-1,-1,-1), \alpha=0.55$} & \multicolumn{2}{c|}{$x_{0}=(0,0.5,0), \alpha=0.55$} & \multicolumn{2}{c|}{$x_{0}=(0,0.5,0), \alpha=2 / 3$} \\
\hline DOFs & L2-error & rate & L2-error & rate & L2-error & rate \\
\hline 316 & 0.00073994 & - & 0.00069287 & - & 0.00074102 & - \\
3.032 & 0.00016401 & 2.00 & 0.00023565 & 1.43 & 0.00023 & 1.55 \\
26.416 & $3.9176 \mathrm{e}-05$ & 1.98 & $6.8242 \mathrm{e}-05$ & 1.72 & $6.2591 \mathrm{e}-05$ & 1.80 \\
22.0256 & $9.6835 \mathrm{e}-06$ & 1.98 & $1.9077 \mathrm{e}-05$ & 1.80 & $1.6589 \mathrm{e}-05$ & 1.88 \\
1.798 .336 & $2.4305 \mathrm{e}-06$ & 1.97 & $5.2412 \mathrm{e}-06$ & 1.85 & $4.3418 \mathrm{e}-06$ & 1.92 \\
14.532 .992 & $6.1407 \mathrm{e}-07$ & 1.98 & $1.44225 \mathrm{e}-06$ & 1.87 & $1.1264 \mathrm{e}-06$ & 1.94 \\
\hline
\end{tabular}

Table E.16 Fichera cube 


\section{References}

1. Adams, R.A.: Sobolev Spaces. Academic Press (1975)

2. Apel, T., Pfefferer, J., Rösch, A.: Finite element error estimates for Neumann boundary control problems on graded meshes. Comput. Optim. Appl. 52(1), 3-28 (2012). DOI 10.1007/s10589-011-9427-x. URL http://dx.doi.org/10.1007/s10589-011-9427-x

3. Apel, T., Pfefferer, J., Rösch, A.: Finite element error estimates on the boundary with application to optimal control. Math. Comp. 84, 33-80 (2015)

4. Babuška, I., Osborn, J.: Eigenvalue problems. In: Handbook of Numerical Analysis, Vol. II, pp. 641-789. North Holland (1991)

5. DeVore, R., Lorentz, G.: Constructive Approximation. Springer Verlag (1993)

6. Evans, L.: Partial Differential Equations. American Mathematical Society (1998)

7. Evans, L., Gariepy, R.: Measure Theory and Fine Properties of Functions. CRC Press (1992)

8. Flemisch, B., Melenk, J.M., Wohlmuth, B.I.: Mortar methods with curved interfaces. Appl. Numer. Math. 54(3-4), 339-361 (2005). DOI 10.1016/j.apnum.2004.09.007. URL http://dx.doi.org/10.1016/j.apnum.2004.09.007

9. Gilbarg, D., Trudinger, N.S.: Elliptic Partial Differential Equations of Second Order. Grundlagen der mathematischen Wissenschaften 224. Springer (1977)

10. Grisvard, P.: Elliptic Problems in Nonsmooth Domains. Pitman (1985)

11. Horger, T., Melenk, J., Wohlmuth, B.: On optimal $L^{2}$ - and surface flux convergence in FEM (extended version). Tech. rep. (2015). URL http://arxiv.org/abs/1501.03003

12. Khoromskij, B., Melenk, J.: Boundary concentrated finite element methods. SIAM J. Numer. Anal. 41(1), 1-36 (2003)

13. Larson, M., Massing, A.: $L^{2}$-error estimates for finite element approximations of boundary fluxes. Tech. rep., http://arxiv.org/abs/1401.6994 (2014). URL http://arxiv.org/abs/1401.6994

14. Li, J., Melenk, J.M., Wohlmuth, B., Zou, J.: Optimal a priori estimates for higher order finite elements for elliptic interface problems. Appl. Numer. Math. 60(1-2), 19-37 (2010). DOI 10.1016/j.apnum.2009.08.005. URL http://dx.doi.org/10.1016/j.apnum.2009.08.005

15. Melenk, J.: $h p$ finite element methods for singular perturbations, Lecture Notes in Mathematics, vol. 1796. Springer Verlag (2002)

16. Melenk, J., Praetorius, D., Wohlmuth, B.: Simultaneous quasi-optimal convergence rates in FEM-BEM coupling. Math. Meth. Appl. Sci. (2014). DOI 10.1002/mma.3374

17. Melenk, J., Rezaijafari, H., Wohlmuth, B.: Quasi-optimal a priori estimates for fluxes in mixed finite element methods and applications to the Stokes-Darcy coupling. IMA J. Numer. Anal. 34(1), 1-27 (2014)

18. Melenk, J., Wohlmuth, B.: Quasi-optimal approximation of surface based Lagrange multipliers in finite element methods. SIAM J. Numer. Anal. 50, 2064-2087 (2012)

19. Morrey, C.: Multiple Integrals in the Calculus of Variations. Springer Verlag (1966)

20. Scott, L., Zhang, S.: Finite element interpolation of nonsmooth functions satisfying boundary conditions. Math. Comp. 54, 483-493 (1990)

21. Tartar, L.: An introduction to Sobolev spaces and interpolation spaces, Lecture Notes of the Unione Matematica Italiana, vol. 3. Springer, Berlin (2007)

22. Triebel, H.: Interpolation theory, function spaces, differential operators, second edn. Johann Ambrosius Barth, Heidelberg (1995)

23. Wahlbin, L.: Local behavior in finite element methods. In: P. Ciarlet, J. Lions (eds.) Handbook of numerical Analysis. Volume II: Finite element methods (Part 1), pp. 353522. North Holland (1991)

24. Wahlbin, L.: Superconvergence in Galerkin finite element methods, Lecture Notes in Mathematics, vol. 1605. Springer Verlag (1995)

25. Waluga, C., Wohlmuth, B.: Quasi-optimal a priori interface error bounds and a posteriori estimates for the interior penalty method. SIAM J. Numer. Anal. 51(6), 3259-3279 (2013). DOI 10.1137/120888405. URL http://dx.doi.org/10.1137/120888405 
Noname manuscript No.

(will be inserted by the editor)

\title{
Insert your title here
}

Do you have a subtitle?

If so, write it here

First Author • Second Author

Received: date / Accepted: date

\begin{abstract}
Insert your abstract here. Include keywords, PACS and mathematical subject classification numbers as needed.
\end{abstract}

Keywords First keyword · Second keyword · More

\section{Introduction}

Your text comes here. Separate text sections with

\section{Section title}

Text with citations 2 and [1.

2.1 Subsection title

as required. Don't forget to give each section and subsection a unique label (see Sect. 2).

Paragraph headings Use paragraph headings as needed.

$$
a^{2}+b^{2}=c^{2}
$$

\footnotetext{
F. Author

first address

Tel.: +123-45-678910

Fax: +123-45-678910

E-mail: fauthor@example.com

S. Author

second address
} 


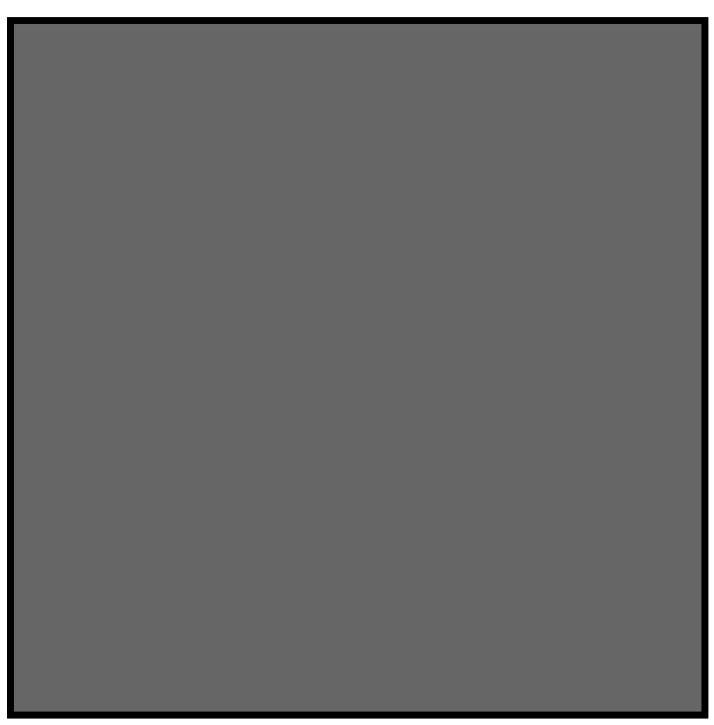

Fig. 1 Please write your figure caption here

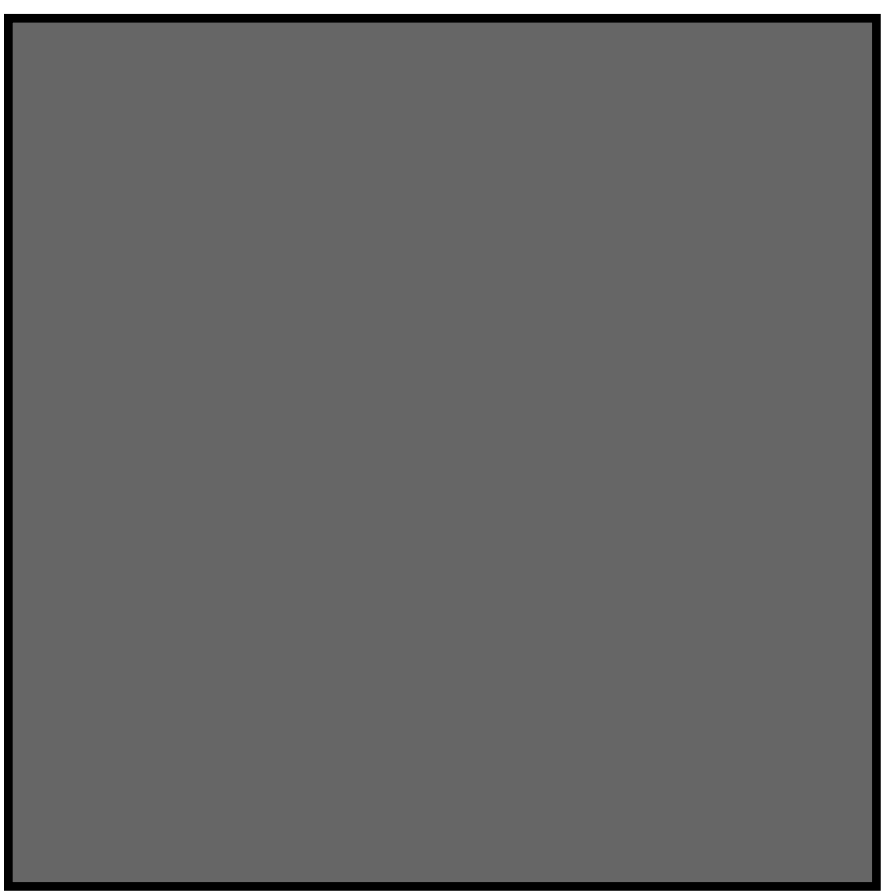

Fig. 2 Please write your figure caption here 
Table 1 Please write your table caption here

\begin{tabular}{lll}
\hline first & second & third \\
\hline number & number & number \\
number & number & number
\end{tabular}

\section{References}

1. Author, Article title, Journal, Volume, page numbers (year)

2. Author, Book title, page numbers. Publisher, place (year) 